\title{
Abstracts
}

\section{SGP Congress 2019}

\section{Montreux, Switzerland, 9-10 May 2019}

\section{Poster Sessions}

\section{Poster Discussion 1: Infectious and Interstitial Diseases}

\author{
P01 \\ Whole Genome Sequencing Characterization of \\ Isoniazid Resistance Mechanisms in Mycobacterium \\ tuberculosis Undetected by Conventional Molecular \\ Methods \\ S. Laurent ${ }^{1}$, F. Zakham ${ }^{1}$, C. Bertelli ${ }^{1}$, L. Merz ${ }^{2}$, L. Nicod ${ }^{3}$, \\ J. Mazza-Stalder ${ }^{3}$, G. Greub ${ }^{1}$, K. Jaton ${ }^{1}$, O. Opota ${ }^{1}$ \\ ${ }^{1}$ Institute of Microbiology, University of Lausanne, University \\ Hospital of Lausanne, ${ }^{2}$ Établissements Hospitaliers du Nord \\ Vaudois Site de St Loup, St Loup, ${ }^{3}$ Department of Pneumology, \\ Lausanne University Hospital, Lausanne, Switzerland, Lausanne, \\ Switzerland
}

Background: We report the delay in the introduction of a complete efficient anti-tuberculous drug regimen in a patient due to a Mycobacterium tuberculosis strain displaying a high level of resistance to Isoniazid (INH), undetected by conventional molecular targeted approach. We aimed to characterize the resistance mechanism of this isolate and to assess the occurrence and significance of such mechanism.

Methods: We used Sanger amplicon sequencing approach as well as whole genome sequencing (WGS) to characterize the INH- resistance isolate. Furthermore, we conducted a large WGS-based study to assess the occurrence and the clinical significance of such resistance mechanism using publicly available WGS.

Results: Sanger sequencing did not reveal any known mutation in $k a t G$ nor inh $A$ in this INH-R isolate. WGS identified a large loss of function insertion $(>1,000 \mathrm{pb})$ in $k a t G$ together with an $o x y R$ $a h p C$ mutation $-57 \mathrm{C}>\mathrm{T}$ known to rescue kat $G$ function, previously described as markers of INH-R. A total of 2398 genomes were analyzed, including 1364 INH-S and 1034 INH-R. Among the 1034 INH-R isolates, 915/1034 had katG Ser315 and/or inhA $-15 \mathrm{G}$ mutations. Eighteen INH-R isolates presented low frequency oxyR-ahpC mutation; among them 7/18 specimen displayed a $k a t G$ loss of function mutations (frameshifts, early stops or structural variants near $k a t G$ ). The eleven remaining INH-R strains had mutations in $k a t G$ of unknown significance. Low frequency, putative compensatory oxyR-ahpC mutations were found in both INH-R and INH-S datasets, questioning their diagnostic relevance for resistance prediction.

Conclusions: This study demonstrates that some INH-R mechanisms due to kat $G$ loss of function mutations associated with high MICs may remain undetected by targeted molecular approaches but could be detected by WGS mapping. This study improves the understanding of the association between $o x y R-a h p C$ compensatory mutations, and structural variation in kat $G$ suggesting that screening for oxyR-ahpC compensatory mutations could be beneficial, but should always be treated with caution.

Disclosure: Nothing to disclose.

\section{KARGER}

E-Mail karger@karger.com www.karger.com/res (c) 2019 Swiss Respiratory Society SGP.

Published by S. Karger AG, Basel.

All rights reserved. 


\section{P02 \\ The Use of Broad-Range Pan-Mycobacterial PCR in the Diagnosis of Nontuberculous Mycobacteria Infections: A 10-Years Retrospective Study}

\author{
S. Andenmatten ${ }^{1}$, O. Opota ${ }^{1}$, J. Mazza-Stalder' ${ }^{2}$, L. Nicod², \\ G. Greub ${ }^{1}$, K. Jaton 1
}

${ }^{1}$ Institute of Microbiology, University of Lausanne and University Hospital Center, Lausanne, Switzerland, ${ }^{2}$ Division of Pulmonology, University of Lausanne and University Hospital of Lausanne, Lausanne, Lausanne, Switzerland

Background: During the last decades, the diagnosis of mycobacterial infections has been improved by the development of molecular methods aimed to circumvent the slow or difficult growth of these organisms, hence reducing the time to diagnosis from weeks to days as compared to culture. The broad range pan-mycobacterial PCR can detect all the mycobacterial species directly from clinical specimens. We aimed to evaluate its usefulness and clinical added value for the diagnosis of non-tuberculosis mycobacteria (NTM) infections.

Methods: We performed, a retrospective study including 955 samples taken from 639 patients with clinical suspicion of pulmonary or extra-pulmonary infection with nontuberculosis mycobacteria over a 10 years period (2003-2013). The performance of the pan-mycobacterial PCRs, smear microscopy and culture were established using a composite reference standard culture and clinical data. We also compared the time to microbial diagnosis between the direct pan-mycobacterial PCR and culture.

Results: The sensitivity, specificity, positive and negative predictive values of the pan-mycobacterial PCR were 61.9\% (53.869.4), 99.1\% (98.2-99.6), 92.9\% (86-96.5) and 93.4\% (91.6-94.9) respectively, when considering all specimens. When considering smear positive specimens and smear negative specimens, the sensitivity was $81.8 \%$ and $40 \%$ respectively. The sensitivity for pulmonary and extra-pulmonary smear positive specimens was $85.2 \%$ versus $73.9 \%$. The median time to identification at species level was 6 days (Standard deviation [SD], 2.67) for the pan-mycobacterial PCR (when positive) and 35 days (SD, 17.67) for culture, which represents a 29 days shorter time to results $(\mathrm{p}<0.0001)$.

Conclusion: The pan-mycobacterial PCR displays a substantial benefit in term of time to diagnose nontuberculous infections when compared to culture. Despite an excellent specificity, its sensitivity is yet limited in particular for smear negative specimens, which might be improved by relying to real-time PCRs.

Disclosure: Nothing to disclose.

\section{P03 \\ Lower Airway Bacteria and Anelloviruses Reveal Ecological Disturbance Linked to Function Decline in the Transplanted Lung}

\author{
E. Bernasconi ${ }^{1}$, D.-A. Wurlod ${ }^{1}$, J.-D. Aubert ${ }^{1,2}$, A. Koutsokera ${ }^{1}$, \\ C. Pattaroni 1,3 , A. Rapin 1 , L. Mercier ${ }^{1}$, B.J. Marsland ${ }^{1,3}$, L.P. Nicod ${ }^{1}$ \\ ${ }^{1}$ Service de Pneumologie, ${ }^{2}$ Centre de Transplantation \\ d'Organes, Centre Hospitalier Universitaire Vaudois (CHUV), \\ Lausanne, Switzerland, ${ }^{3}$ Department of Immunology and \\ Pathology, Central Clinical School, Monash University, \\ Melbourne, VIC, Australia
}

Background: Physiologic functions in the healthy lung depend on immune homeostasis, to which the contribution of host-microbe interactions is currently ill-defined. Lung transplant recipients may develop either reversible (e.g. infection) or irreversible (Chronic Lung Allograft Dysfunction, CLAD) pulmonary function decline. We hypothesized that disturbance in lung bacterial and viral communities associate with a breakdown of innate immune homeostasis and impaired lung function.

Methods: DNA and RNA were isolated from 135 bronchoalveolar lavages (BAL) obtained from 36 patients up to 24 months post-transplantation. Bacterial communities were analyzed using $16 \mathrm{~S}$ sequencing and qPCR. The loads of highly prevalent anelloviruses (alpha, beta and gamma torque virus) and levels of host gene expression (set of 31 targets involved in host-microbe interactions) were quantified using qPCR. Associations with spirometry data were explored.

Results: Transversal analysis of our patient cohort revealed limited associations between microbiota features (load, composition and structure) and spirometry data. However, we observed marked shifts in bacterial and/or viral communities in a subset of patients with close longitudinal follow-up that preceded or coincided with reversible lung function decline or CLAD. These manifestations of lung ecological disturbance were further reflected in host gene expression signatures, with a particular trend towards inflammation and degradative remodelling (matrix metalloproteinase-7, -9 and -12), and higher BAL neutrophilia.

Conclusion: There is a strong temporal link between disturbance of microbial communities and function decline in the transplanted lung. Tracking early signs of microbiota dysbiosis has potential for predicting lung pathophysiology following transplantation, and identifying those patients with a trajectory towards CLAD.

Disclosure: Nothing to disclose. 


\section{P04 \\ Comorbidity Clusters Associated with Length of Hospital Stay in Patients with Tuberculosis}

\section{F. Baty, O. Schoch, S. Tonko, M. Brutsche}

Lung Center, Cantonal Hospital St. Gallen, St. Gallen, Switzerland

Introduction: Tuberculosis (TB) is a global public health threat with significant burden to society and healthcare system. Outpatient treatment and shorter hospital stays may reduce costs. TBassociated length of hospital stay (LOS) is heterogeneous and it is of interest to better understand risk factors associated with longer LOS. The aim of this study was to investigate the relationship between comorbidities and LOS in patients hospitalized for TB as primary diagnosis.

Methods: A Swiss-wide hospitalization database provided by the Swiss Federal Office for Statistics was used. Month and year of admission, gender, age, LOS and in-hospital mortality were extracted for all hospitalization cases with TB as primary diagnosis and compared with controls matched for age, gender and admission date. Information about type of TB, age and comorbidities (up-to 50 co-diagnoses coded using ICD-10) were analyzed.

Results: Over the period 2002-2015, the median LOS of 7392 patients hospitalized primarily for TB was stable at 14 days [IQR: 6-22 days] (Fig. 1a) and was longer in miliary TB and older patients. TB-associated comorbidities included HIV, liver diseases, anemia, malnutrition and diseases of the genitourinary system (Fig. 1b). Longer LOS was strongly associated with 3 clusters of comorbidity (Fig. 1c). 1) Malnutrition, cachexia and anemia (median LOS: 20 days [IQR: 13-31 days], $\mathrm{p}<0.001)$; 2) Toxic liver disease and hepatitis (median LOS: 23 days [IQR: 13-39 days], $\mathrm{p}<0.001$ ); 3) Antibiotic resistance to betalactam (median LOS: 29 days [IQR: 19-59.5 days], $\mathrm{p}<0.001)$.

Conclusion: We identified clusters of comorbidities significantly associated with longer LOS in patients with TB. Comorbidities are relevant determinants for prolonged hospital stays in TB.

Disclosure: Nothing to disclose.

\section{P05 \\ Multidimensional Genomic Mapping Reveals the Pathophysiology of Mycobacterium abscessus}

L.Boeck ${ }^{1}$, M. Skwark ${ }^{2}$, W. Pearson ${ }^{3}$, S. Burbaud ${ }^{2}$, J. Sangen ${ }^{2}$, S. Malhotra ${ }^{2}$, J. Bryant ${ }^{2}$, T.L. Blundell ${ }^{2}$, M. Dionne ${ }^{3}$, J. Parkhill' , A. Floto ${ }^{2}$

${ }^{1}$ Department of Biomedicine, Universität Basel, Basel, Switzerland, ${ }^{2}$ University of Cambridge, Cambridge, ${ }^{3}$ Imperial College London, London, ${ }^{4}$ Wellcome Trust Sanger Institute, Hinxton, United Kingdom

Mycobacterium abscessus (MAB) is an emerging pathogen, particularly for individuals with cystic fibrosis $(\mathrm{CF})$ and has become the most lethal and frequent multidrug-resistant mycobacterial infection in the developed World. M. abscessus infections are increasingly common in CF cohorts, are difficult and sometimes impossible to treat despite prolonged combination antibiotic therapy, and compromise safe lung transplantation. Very little is known about the genetic determinants controlling M. abscessus infection, virulence, and antimicrobial resistance, or how mycobacterial and non-mycobacterial genes (derived from horizontal gene transfer) interact to influence pathophysiology. We therefore sought to define the critical pathways regulating $M$. abscessus biology through multidimensional genomic mapping.

We used whole genome sequence data from 2266 respiratory isolates of $M$. abscessus obtained from individuals with CF to map epistatic interactions (using correlation compressed direct coupling analysis; ccDCA). A subset of 350 isolates were phenotyped across multiple dimensions including: antibiotic resistance and tolerance; carbon source utilisation; macrophage infection and death; and host survival and immune response during in vivo Drosophila infection. Experimental and clinical phenotypic data was examined for clustering (using t-distributed stochastic neighbour embedding; t-sne), and mapped to genomic sequences (by combining computational structural predictions and linear mixed model GWAS). Selected hits were then validated using CRISPRi.

Our study revealed key functional networks controlling the pathophysiology of $M$. abscessus. For example, we identified known and new determinants of resistance to amikacin, clarithromycin, clofazimine, cefoxitin and linezolid, and defined virulence in Drosophilainfection models as a heritable trait which clusters with clinical outcomes in infected patients.

Disclosure: Nothing to disclose.

\section{P06 \\ The Impact of Inpatient Pulmonary Rehabilitation on Functional Independence in Patients with Interstitial Lung Disease}

S.A.Guler ${ }^{1}$, L. Bovet ${ }^{2}$, P. Brun ${ }^{2}$

'Department of Pulmonary Medicine, University Hospital Bern (Inselspital), Bern, ${ }^{2}$ Berner Reha Zentrum Heiligenschwendi, Heiligenschwendi, Switzerland

Introduction: Regaining independence in functions of daily living is a key goal of inpatient pulmonary rehabilitation (IPR). We aimed to quantify the effect of IPR on functional independence in patients with interstitial lung disease (ILD), to identify influential factors, and specifically to determine the importance of change in 6-minute walk distance (6MWD) for functional independence.

Methods: Baseline characteristics were collected retrospectively from consecutive patients with ILD who attended a 3-week IPR program; 6MWD and the functional independence measure (FIM) were collected at IPR admission and discharge. The FIM contains 13 motor and 5 cognitive items (each 1-7 points) with higher scores denoting higher levels of independence.

Results: We included 33 men and 17 women with ILD (17 with idiopathic pulmonary fibrosis). Men were older than women (mean [standard deviation, SD] 73.9 [10.8] versus 67.2 [17.1] years, 


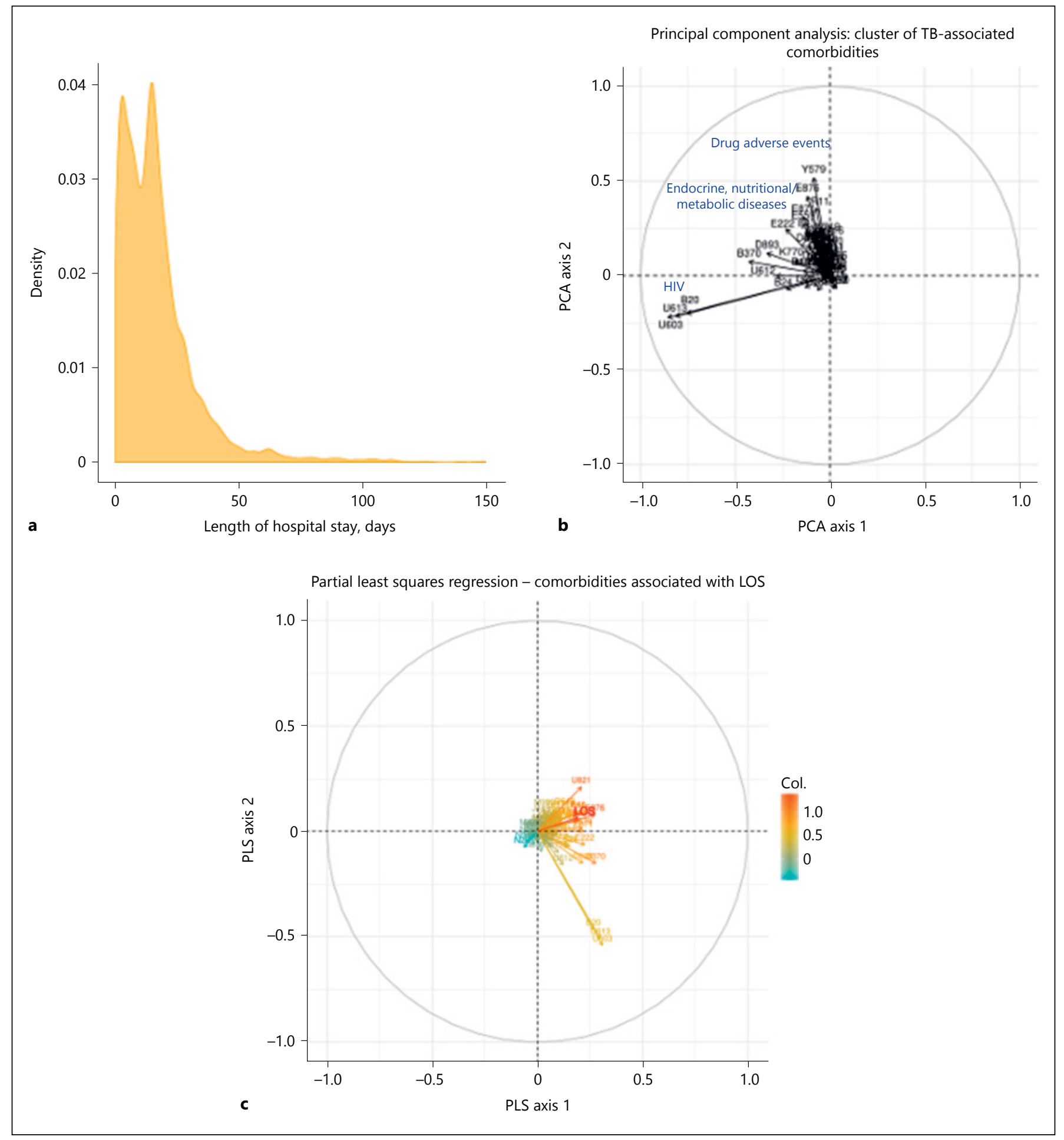

Fig. 1. Relationship between TB-associated comorbidities and length-of-hospital stay (for Abstract no P04). 


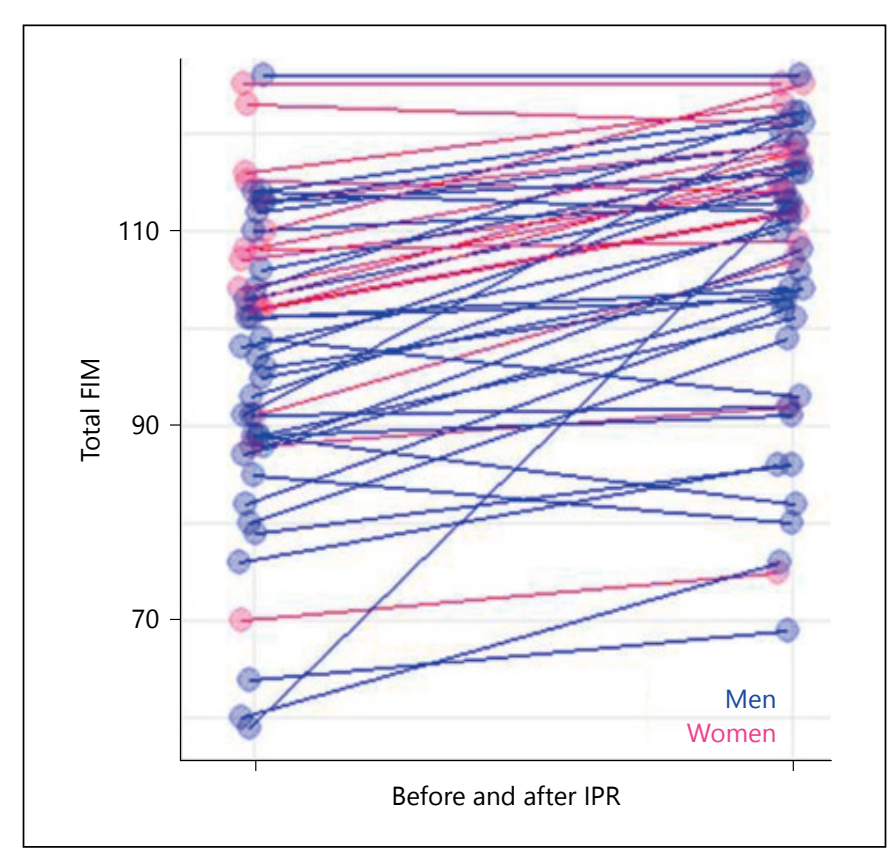

Fig. 1. (for Abstract no P06).

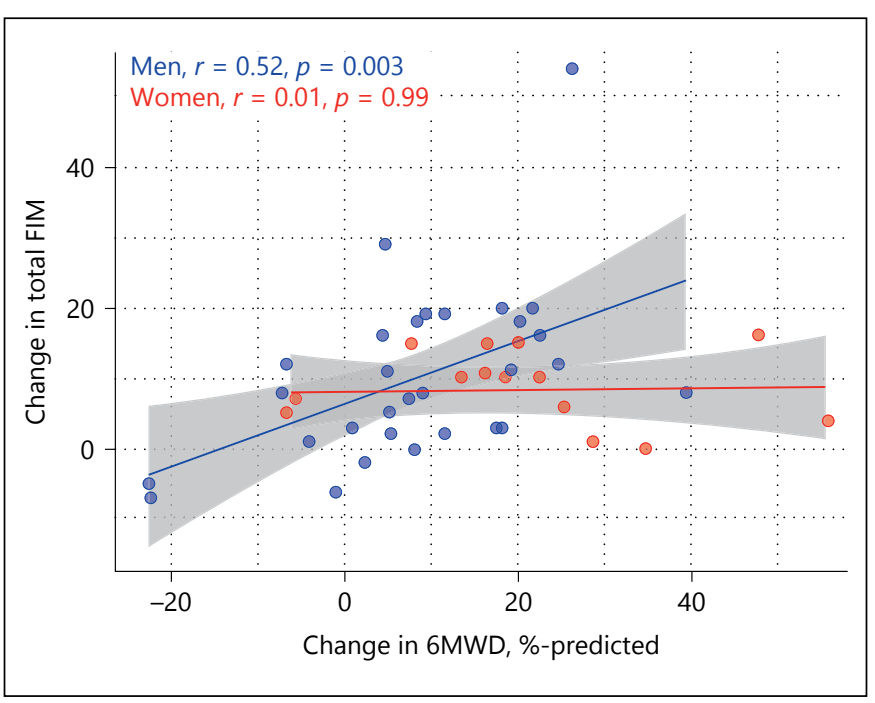

Fig. 2. (for Abstract no P06).

$\mathrm{p}=0.04)$, patients had moderately reduced forced vital capacity (mean [SD] 64.9 [19] \%-predicted), and severely reduced diffusing capacity for carbon monoxide (mean [SD] 37.1 [13] \%-predicted). At baseline mean (SD) 6MWD was 215 (111) meters/43 (20) $\%$-predicted, with significant increase after IPR (mean difference [95\%-confidence interval, CI] 45 [30-61] meters, $\mathrm{p}<0.001)$. FIM increased after IPR (mean difference [95\% CI] 9.24 [6.4-12.1], p < 0.001 , Figure 1). On unadjusted analysis gain in $6 \mathrm{MWD}$ correlated significantly with gain in FIM, particularly in men (Figure 2). The odds of a substantial gain in FIM (highest quartile) increased significantly with larger increase in 6MWD during IPR (OR 1.08 per \%-6MWD-predicted, 95\% CI 1.01-1.19, p = 0.04), including with adjustment for age and sex.

Conclusion: IPR effectively improves functional independence in patients with ILD. The increase of 6MWD during IPR, which is a commonly used surrogate for a successful pulmonary rehabilitation, correlates significantly with gain in FIM, suggesting that the improvement of physical performance translates into more independence in daily life.

Disclosure: Nothing to disclose.

\section{P07 \\ Clinical Predictors to Improve Value of High Resolution Chest Tomography for Diagnosis of Usual Interstitial Pneumonia Pattern on Histopathology}

\author{
A. Kharat $^{1}$, J. Plojoux ${ }^{1}$, B. Bedat ${ }^{2,3}$, X. Montet $^{3,4}$, W. Karenovics ${ }^{2}$, \\ J.-C. Pache ${ }^{3,5}$, K.E. Hostettler 6 , C. Von Garnier ${ }^{7}$, P.M. Soccal ${ }^{1,3}$, \\ D. Adler ${ }^{1,3}$
}

${ }^{1}$ Division of Pneumology, Geneva University Hospitals, ${ }^{2}$ Division of Thoracic Surgery, Geneva University Hospitals, ${ }^{3}$ Faculty of Medicine, Geneva University, ${ }^{4}$ Division of Radiology, Geneva University Hospitals, ${ }^{5}$ Department of Clinical Pathology, Geneva University Hospitals, Geneva, ${ }^{6}$ Pneumology and Biomedicine Department, Universitätsspital Basel, Basel, ${ }^{7}$ Respiratory Medicine Department, Standort Tiefenau und Inselspital Bern, Bern, Switzerland

Background: There are conditional recommendations for lung biopsy after multidisciplinary discussion (MDD) to diagnose usual interstitial pneumonia (UIP) in patients with non-definitive UIP pattern on high resolution chest tomography (HRCT). Yet, many patients are unfit for lung biopsy or decline because of personal preferences. The objectives of this pilot study are 1) to determine characteristics of possible UIP pattern on HRCT to predict biopsyproven UIP and 2) to force in a model clinical variables in addition to HRCT features to predict biopsy-proven UIP.

Method: 25 consecutive patients from a single academic centre with biopsy-proven (cryobiopsy $n=11$, surgical lung biopsy $\mathrm{n}=14$ ) interstitial lung disease and non-definitive UIP pattern on HRCT after MDD. Sensitivity, specificity, positive and negative predictive values of HRCT were computed using biopsy as gold standard. Age, gender, smoking history (pack-years) and "possible UIP" pattern on HRCT were forced in a logistic model to improve the performance of HRCT.

Results: 12/25 (48\%) had possible UIP on HRCT. 16/25 (64\%) had biopsy-proven UIP. Possible UIP pattern had a sensitivity of $63 \%$ [95\% CI 35-85] and a PPV of $83 \%$ [95\% CI 52-98] for biopsy-proven UIP. Addition of clinical variables ([age (yrs)*0.15+ $0.05^{*}$ pack-years + male gender* $1+$ possible UIP pattern*1.35]-8) in a logistic model improved HRCT performance: AUC 0.85 [95\% CI $0.64-0.98$ ]. With a cut-off value of 3 , sensitivity of the model 


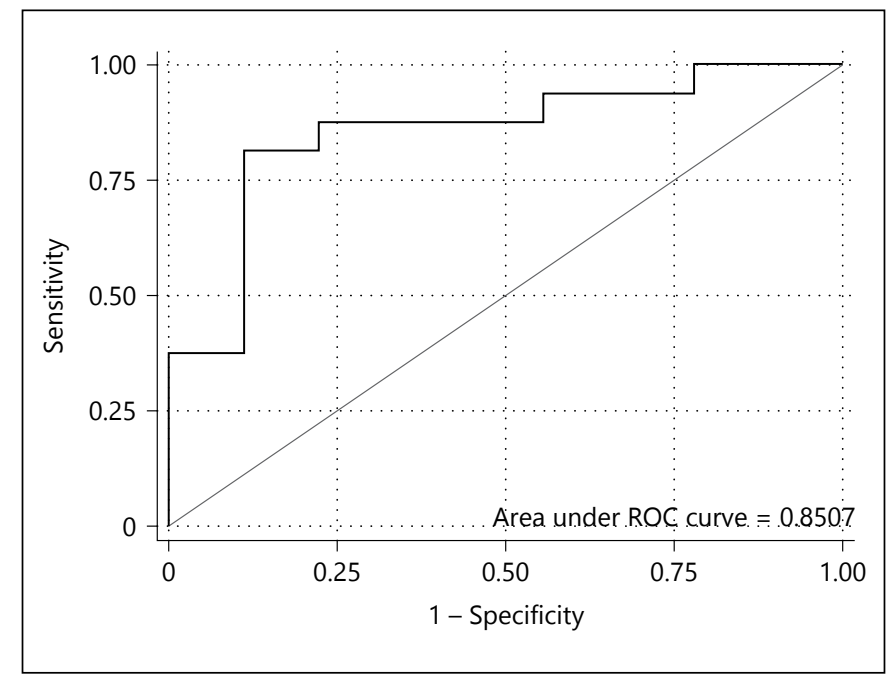

Fig. 1. ROC curve of a logistic model combining HRCT features and clinical variables to predict biopsy-proven UIP (for Abstract no P07).

improved to 88\% [95\% CI 61-98] without major difference in PPV (78\% [95\% CI 52-94]).

Conclusion: In our experience with ILD patients, combining HRCT possible UIP pattern with clinical variables can improve detection of biopsy-proven UIP. Our findings could facilitate discussion with patients declining or unfit for lung biopsy. Precision interval of our model is still too large to change clinical decisionmaking, probably due to small sample size. Our results also highlight the need for a well-designed national coordinated research on ILD in Switzerland.

Disclosure: Nothing to disclose.

\section{P08 \\ Electroporation-Mediated Overexpression of Hepatocyte Growth Factor Results in Accumulation of Bone Marrow Mesenchymal Stem Cells to the Injured Lung}

K. Fytianos ${ }^{1,2}$, R. Schliep ${ }^{3}$, L. Knudsen ${ }^{3}, \underline{\text { A. Gazdhar }}^{1,2}$, T. Geiser $^{1,2}$

${ }^{1}$ Pneumology, Bern University Hospital, ${ }^{2}$ Department of Bio-Medical Research, University of Bern, Bern, Switzerland, ${ }^{3}$ Institute of Anatomy, Hannover Medical School, Hannover, Germany

Introduction: Idiopathic Pulmonary Fibrosis (IPF) is a terminal lung disease with complex pathophysiology. Two approved anti-fibrotic drugs slow the disease progression but there is no cure. Hepatocyte Growth Factor (HGF) and bone marrow derived stem cells (BMSC) have been proven to have anti-fibrotic properties. However, the mechanisms are not fully known, specially the effect of HGF on BMSC migration and engraftment into fibrotic lung tissue. We hypothesize that HGF overexpression in the lung increases BMSC migration and engraftment to bleomycin injured lung and help in alveolar epithelial repair and regeneration.

Methods: Adult male rats were intratracheally instilled with bleomycin to induce lung injury and fibrosis. 5 days later, the proximal end of the right femur was drilled and pre-labelled BMSC were injected. 2 days after surgery, in vivo electroporation mediated gene transfer of HGF or the empty vector (EV) was performed. Animals were sacrificed 3 days after gene transfer. SDF-1 levels were determined by ELISA.

Results: We report that HGF treated animals show significantly increased numbers of BMSC in the injured lung compared to controls, as shown by confocal microscope and 2-Photon microscopy. Flow cytometry analysis revealed $15687 \pm 122$ pre-labelled BMSC in HGF treated lungs vs $10282 \pm 1562$ in EV control group $(\mathrm{p}<0.05)$. Apart from elevated numbers of BMSCs in HGF-treated animals, elevated levels of Stromal-Derived Factor 1 (SDF-1) in vivo were observed. (705.5 \pm 54.7 pg vs $282.3 \pm 54.7$ pg SDF- 1 in EV control, $\mathrm{p}<0.01)$ Moreover, upregulation of p21-ActivatedKinases (PAKs) was observed when BMSC were treated with HGF in vitro.

Conclusions: Overexpression of HGF in the bleomycin-treated lung results in enhanced numbers of BMSC. Moreover, SDF-1 levels are enhanced in HGF-treated lungs with bleomycin-induced injury, assuming that increased BMSC numbers are due to a SDF-1 increase.

Disclosure: Nothing to disclose.

\section{P09 \\ Transcriptomic Profiling of Potential Therapeutic Target Cell Populations in Idiopathic Pulmonary Fibrosis}

\author{
M. Boesch' ${ }^{1}$, P. Kahn ${ }^{2}$, F. Baty ${ }^{1}$, L. Fang ${ }^{2}$, M. Tamm², M.H. Brutsche ${ }^{1}$, \\ K.E. Hostettler ${ }^{2}$ \\ ${ }^{1}$ Lungenzentrum, Kantonsspital St. Gallen, St. Gallen, \\ ${ }^{2}$ Department of Biomedicine, University Hospital Basel, Basel, \\ Switzerland
}

Introduction: Idiopathic pulmonary fibrosis (IPF) is a lethal disease characterized by ongoing damage to the alveolar epithelium, the repair of which triggers a secondary fibrotic response in lung-resident fibroblasts impinging on lung function. Novel treatment concepts for IPF are desirable and include cell-based modalities, such as adoptive transfer of naturally anti-fibrotic mesenchymal stem cells (MSCs). We previously showed that lung-resident MSCs can undergo mesenchymal-to-epithelial transition (MET) in vitro, raising the possibility of epithelial repair by MSCs. Here, we profiled the transcriptome of MSCs and MSC-derived epithelial cells in IPF versus non-IPF samples.

Methods: Primary lung MSCs were recovered from patients with interstitial lung diseases (IPF and non-IPF). MET was induced in fractions of these cells through addition of an epithelial-specific culture medium and the epithelial identity of the MSC-derived cells was confirmed using microscopy and immunofluorescence. 
The transcriptome of MSCs and MSC-derived epithelial cells was comprehensively analysed using RNA sequencing.

Results: Primary MSCs from IPF versus non-IPF sources exhibited largely overlapping transcriptomes, with only 11 genes differentially regulated including COL1A2 and ADAMTS9. Conversely, transitioned epithelial cells derived from IPF versus nonIPF MSCs showed significant differences in their transcriptomic landscape, with a total of 177 differentially expressed genes including keratins (KRT7, KRT75), mucins (MUC4, MUC21), cell adhesion molecules (L1CAM, ICAM1), immune-related factors (CSF2, IL7R) and matrix metalloproteinases (MMP10). Moreover, we observed marked enrichment of proliferation-associated pathways during the process of MET, whereas cytokine signalling was downregulated.

Conclusion: RNA sequencing revealed the transcriptomic landscape of primary MSCs and their MET-transitioned progeny from both IPF and non-IPF sources, thereby pinpointing diseasespecific differences in the underlying pathomechanisms. The stage is set to use these high-content data to decipher the functional role and potential therapeutic use of lung-resident MSCs and MSCderived epithelial cells for anti-fibrosis and epithelial repair in the fibrotic lung, respectively.

Disclosure: Nothing to disclose.

\section{P10 \\ Effect of Acetazolamide on Pulmonary Hemodynamics in COPD-Patients Going to Altitude: A Randomized Trial}

\author{
M. Lichtblau ${ }^{1}$, F. Michael', L. Mayer ${ }^{1}$, A. Buergin ${ }^{1}$, P.M. Scheiwiller', \\ S. Saxer ${ }^{1}$, U. Sheraliev' ${ }^{2}$, M. Mademilov' ${ }^{2}$, P.R. Bader' ${ }^{1}$, S.R. Schneider', \\ F. Tanner ${ }^{3}$, T. Sooronbaev ${ }^{2}$, K.E. Bloch' ${ }^{1}$, S. Ulrich ${ }^{1}$ \\ ${ }^{1}$ Klinik für Pneumologie, UniversitätsSpital Zürich, Zürich, \\ Switzerland, ${ }^{2}$ National Center for Cardiology and Internal \\ Medicine, Bishkek, Kyrgyzstan, ${ }^{3}$ Klinik für Kardiologie, \\ UniversitätsSpital Zürich, Zürich, Switzerland
}

Background: Chronic obstructive pulmonary disease (COPD) may predispose to symptomatic pulmonary hypertension at high altitude. We investigated whether preventive acetazolamide treatment would attenuate the altitude-induced increase in pulmonary artery pressure (PAP) in lowlanders with COPD ascending to $3,100 \mathrm{~m}$.

Methods: In this placebo-controlled, double-blind trial, nonhypercapnic COPD patients GOLD 2-3 living $<800 \mathrm{~m}$, were randomized to receive either acetazolamide (125-0-250 mg/day) or placebo tablets starting $24 \mathrm{~h}$ before ascent to and while staying at $3,100 \mathrm{~m}$ for 48 hours. Echocardiography was performed at $760 \mathrm{~m}$ before medication and after the first night at 3,100 m. PAP (presented as trans-tricuspid pressure gradient), heart rate (HR), cardiac output $(\mathrm{CO})$ and pulse-oximetry $\left(\mathrm{SpO}_{2}\right)$ were compared between groups.

Results: 112 patients, $68 \%$ male, (mean \pm SD) age $57 \pm 8$ y, $\mathrm{FEV}_{1}$ $61 \pm 12 \%$ pred. were included in the analysis. In 54 patients receiving placebo, PAP at 760 and $3,100 \mathrm{~m}$ was $22 \pm 7$ and $30 \pm 10 \mathrm{~mm}$
$\mathrm{Hg}$, in 58 patients receiving acetazolamide corresponding values were $20 \pm 5$ and $24 \pm 7 \mathrm{~mm} \mathrm{Hg}$; treatment effect (mean difference $(95 \% \mathrm{CI})$ ) was -5 ( -9 to -1$) \mathrm{mm} \mathrm{Hg}$ (all $\mathrm{p}<0.05)$.

In the placebo group $\mathrm{CO}, \mathrm{HR}$ and $\mathrm{SpO}_{2}$ at 760 and $3,100 \mathrm{~m}$ were $4.5 \pm 1.1$ and $4.9 \pm 1.0 \mathrm{~L} / \mathrm{min}$ and $69 \pm 11$ and $76 \pm 11 \mathrm{bpm}$, $95 \pm 2$ and $88 \pm 3 \%$ (all $\mathrm{p}<0.05)$. The corresponding values in the acetazolamide were $4.7 \pm 1.2$ and $4.4 \pm 0.9 \mathrm{~L} / \mathrm{min}, 71 \pm 11$ and $74 \pm$ $10 \mathrm{bpm}, 94 \pm 2$ and $90 \pm 3 \%\left(\mathrm{p}<0.05\right.$ for $\mathrm{HR}$ and $\left.\mathrm{SpO}_{2}\right)$. Treatment effects: CO $-0.6(-1.0$ to -0.2$) \mathrm{L} / \mathrm{min}, \mathrm{HR}-5(-8$ to -1$) \mathrm{bpm}$ and $\mathrm{SpO}_{2} 2$ (1 to 3$) \%$.

Conclusions: In lowlanders with COPD travelling to $3,100 \mathrm{~m}$ preventive acetazolamide treatment attenuated the altitude-induced rise in PAP along with an improved oxygenation but decreased $\mathrm{CO}$ and HR.

Disclosure: Nothing to disclose.

\section{Poster Discussion 2: Airway and Sleep Diseases}

\section{P11 \\ Effect of Oxygen Therapy on Nocturnal Breathing and Sleep in Patients with COPD Travelling to High Altitude. Randomized Trial}

\author{
M. Furian, L. Tan, T.D. Latshang, S.S. Aeschbacher, F. Huber, \\ D. Flueck, M. Lichtblau, S. Ulrich, E.D. Hasler, P.M. Scheiwiller, \\ S. Ulrich, K.E. Bloch \\ University Hospital of Zurich, Department of Respiratory \\ Medicine, Sleep Disorders Center, Zurich, Switzerland
}

Purpose: Patients with chronic obstructive pulmonary disease (COPD) may deteriorate while sleeping at high altitude (Latshang et al. Sleep 2018). We evaluated whether oxygen therapy prevents severe deoxygenation, sleep and breathing disturbances in lowlanders with COPD sleeping at $2048 \mathrm{~m}$.

Methods: 32 patients with COPD, mean \pm SD FEV $54 \pm$ $13 \%$ predicted, living $<800 \mathrm{~m}$, participated in this randomized, placebo-controlled, crossover trial. Polysomnographies were performed at $490 \mathrm{~m}$ and during 2 sojourns at $2048 \mathrm{~m}$, one with nocturnal oxygen ( $3 \mathrm{~L} / \mathrm{min}$ via nasal cannula), the other with placebo (ambient air), in randomized order, with a $>2$-weeks washout-period in-between. Co-primary outcomes were mean nocturnal oxygen saturation $\left(\mathrm{SpO}_{2}\right)$ and apnea-hypopnea index (AHI). www. ClinicalTrials.gov NCT02150590.

Results: During the stay at $2048 \mathrm{~m}$ or within $24 \mathrm{~h}$ after descent, $8(26 \%)$ patients using placebo and $1(4 \%, \mathrm{P}<0.001)$ using oxygen had adverse events such as severe hypoxemia $\left(\mathrm{SpO}_{2}<75 \%\right.$ $>30$ min, $\mathrm{n}=4)$, intolerable dyspnea $(\mathrm{n}=2)$, COPD exacerbation $(n=2)$, or non-sustained ventricular tachycardia $(n=1)$. Per protocol analysis in 23 patients revealed at $490 \mathrm{~m} \mathrm{SpO}_{2} 92 \pm 2 \%$, AHI $19.7 \pm 13.9 / \mathrm{h}$, sleep efficiency $81 \pm 11 \%$. At $2048 \mathrm{~m}$, patients 
using placebo had lower $\mathrm{SpO}_{2} 86 \pm 3 \%$, higher AHI $36.1 \pm 27.9 / \mathrm{h}$, and lower sleep efficiency $72 \pm 15 \%$ (all $\mathrm{P}<0.01$ vs.490 m). In patients using nocturnal oxygen, mean differences $(95 \% \mathrm{CI})$ vs. placebo were: $\mathrm{SpO}_{2}+10 \%$ (9 to 11$)$, AHI $-21.7 / \mathrm{h}(-29.3$ to -14.3 ) and sleep efficiency $+7 \%$ (2 to 11 ). Oxygen therapy improved subjective sleep quality (visual analog scale $0-100 \%$, worst to best) by $+9 \%(0$ to $17, \mathrm{P}<0.05)$.

Conclusion: About one quarter of lowlanders with COPD experienced impaired well-being, pronounced hypoxemia, breathing and sleep disturbances at $2048 \mathrm{~m}$. Oxygen treatment significantly mitigated or prevented these adverse effects and can therefore be recommended to tourists with moderate to severe COPD sleeping at high altitude.

Disclosure: Grants: Lunge Zürich, Swiss National Science Foundation.

\section{P12 \\ Fractionated Exhaled Nitric Oxide Is Increased in Viral Exacerbations of COPD}

\author{
D.M. Schumann ${ }^{1}$, K. Kostikas ${ }^{1}$, D. Schilter ${ }^{2}$, R. Louis ${ }^{3}$, L. Grize ${ }^{4}$, \\ M. Tamm ${ }^{1}$, D. Stolz 1 \\ ${ }^{1}$ Clinic of Pneumology and Pulmonary Cell Research, University \\ Hospital Basel, Basel, ${ }^{2}$ Pneumologie-Praxis Quartier Bleu, \\ Lindenhof Hospital Bern, Bern, Switzerland, ${ }^{3}$ Pneumology \\ Department, University of Liege, Liege, Belgium, ${ }^{4}$ Department \\ of Epidemiology and Public Health, Swiss Tropical and Public \\ Health Institute Basel, Basel, Switzerland
}

Introduction: The fraction of exhaled nitric oxide (FeNO) is a marker of eosinophilic and T-helper type-2 cell airway inflammation in asthma. Although nearly one-third of patients with chronic obstructive pulmonary disease (COPD) have eosinophilic airway inflammation, data on FeNO in COPD are inconsistent.

Methods: Data of 450 patients included in the PREVENT study receiving standardized low-dose therapy with LABA/ICS (Budesonide $400 \mathrm{ug} /$ Formoterol $12 \mathrm{ug}$ daily) with scheduled visits half-yearly at stable state and unscheduled visits for exacerbations and upper respiratory tract infections (URTI) were analyzed. Follow up occurred 10 and 21 days after a URTI. FeNO measurements and multiplex viral PCR for 18 viruses in nasopharyngeal swabs were performed at each visit.

Results: 2664 visits (2063 at stable state, 196 at exacerbation and 405 at the follow-up to an URTI) were performed. FeNO significantly increased from stable state to exacerbations $(\mathrm{p}<0.0001)$ and decreased during the URTI follow-up ( $\mathrm{p}<$ 0.0001 ; median 17 [ 11.5 to 26.0 ] to 26 [16 to 44 ] to 18 [13 to 25.5] ppb, respectively). Adjusted analyses including smoking status, MMRC score, CAT score, SGRQ total score and postbronchodilator $\mathrm{FEV}_{1}$ showed significantly higher FeNO levels in patients with viral infection at exacerbation compared with no viral infection (24.6 vs $15.6 \mathrm{ppb} ; \mathrm{p}=0.041$ ). Differences in FeNO between patients with and without virus detection at stable state did not reach statistical significance $(22.0$ vs 20.9 $\mathrm{ppb}, \mathrm{p}=0.069)$. There was no difference in FeNO between pa- tients with and without growth of potential pathogenic bacteria in sputum in the crude or adjusted multivariate analysis. FeNO was similarly increased in moderate and severe AECOPD (29.3 vs $26.8 \mathrm{ppb}, \mathrm{p}=0.66)$.

Conclusion: In a large COPD cohort on standardized low-dose ICS/LABA, COPD exacerbations, particularly the ones caused by a viral infection, are associated with increased FeNO levels. FeNO decreases to stable state values after convalescence.

Disclosure: Nothing to disclose.

\section{P13 \\ Exercise Performance in Patients with COPD at High Altitude. Randomized Placebo-Controlled Trial Evaluating Effects of Acetazolamide}

\author{
M. Furian ${ }^{1}$, R. Kind ${ }^{1}$, A. Buergin' ${ }^{1}$, P.M. Scheiwiller ${ }^{1}$, L. Mayer ${ }^{1}$, \\ S. Schneider ${ }^{1}$, M. Mademilov'2 ${ }^{2}$ B. Emilov' ${ }^{2}$, M. Lichtblau', \\ B. Estebesova ${ }^{2}$, B. Osmonov ${ }^{2}$, L. Muralt ${ }^{1}$, A. Groth ${ }^{1}$, U. Sheraliev ${ }^{2}$, \\ N.H. Marazhapov' ${ }^{2}$, T.M. Sooronbaev ${ }^{2}$, S. Ulrich ${ }^{1}$, K.E. Bloch ${ }^{1}$
}

${ }^{1}$ University Hospital of Zurich, Department of Respiratory Medicine, Sleep Disorders Center, Zurich, Switzerland,

${ }^{2}$ National Center for Cardiology and Internal Medicine, Bishkek, Kyrgyzstan

Objective: Acetazolamide is used to prevent altitude-related illness but its effect on exercise performance has been debated. The current randomized, placebo-controlled, double-blind trial investigates effects of preventive treatment with acetazolamide on exercise performance in patients with chronic obstructive pulmonary disease (COPD) ascending to high altitude.

Methods: $\mathrm{COPD}$ patients, $\mathrm{FEV}_{1} \geq 40$ to $<80 \%$ predicted, living below $800 \mathrm{~m}$, were randomized to acetazolamide (125-0-250 mg/ day) or placebo, starting $24 \mathrm{~h}$ before ascent to and while staying at $3100 \mathrm{~m}$ for $48 \mathrm{~h}$. Patients performed progressive cycling exercise to exhaustion at $760 \mathrm{~m}$, before taking the study drug, and within $4 \mathrm{~h}$ after arrival at $3100 \mathrm{~m}$. Ventilation, gas exchange, heart rate and arterial blood gases were measured. www.ClinicalTrials.gov NCT03177837.

Results Of 176 randomized patients, 1 using acetazolamide and 9 using placebo $(\mathrm{P}<0.001)$ could not perform exercise because of altitude-related illness. Per protocol analysis, including 103 patients with two CPET assessments, revealed: In 53 patients receiving acetazolamide, maximal work rate (Wmax) and oxygen uptake $\left(\mathrm{V}^{\prime} \mathrm{O}_{2} \mathrm{max}\right)$ at 760 and $3100 \mathrm{~m}$ were $105 \pm 40$ and $91 \pm 40$ watts, and $18.0 \pm 6.8$ and $15.5 \pm 6.8 \mathrm{ml} / \mathrm{min} / \mathrm{kg}(\mathrm{P}<0.0013100 \mathrm{vs} .760 \mathrm{~m}$, both instances). Corresponding $\mathrm{Wmax}$ and $\mathrm{V}^{\prime} \mathrm{O}_{2} \max$ in 50 patients receiving placebo were $107 \pm 41$ and $97 \pm 41$ watts, and $18.9 \pm 7.0$ and $17.2 \pm 7.0 \mathrm{ml} / \mathrm{min} / \mathrm{kg}(\mathrm{P}<0.0013100$ vs. $760 \mathrm{~m}$, both instances $)$. Between-group differences (95\% CI) in altitude-induced changes (acetazolamide vs. placebo-group) in Wmax were -3 watts ( -9 to $+3, \mathrm{P}=0.305)$ and in $\mathrm{V}^{\prime} \mathrm{O}_{2} \max -0.8 \mathrm{ml} / \mathrm{min} / \mathrm{kg}(-2.1$ to $+0.5, \mathrm{P}=$ $0.213)$. At end-exercise, acetazolamide mitigated altitude-induced reductions of $\mathrm{PaO}_{2}$ by $0.8 \mathrm{kPa}(0.1$ to $1.3, \mathrm{P}=0.016)$, reduced $\mathrm{pH}$ by $0.04(0.02$ to $0.06, \mathrm{P}<0.001)$ and reduced serum lactic acid by $1.6 \mathrm{mmol} / \mathrm{l}(0.8$ to $2.4, \mathrm{P}<0.001)$. 
Conclusion: Lowlanders with moderate to severe COPD travelling to $3100 \mathrm{~m}$ experienced an altitude-induced reduction in maximal exercise performance by about $10 \%$. Preventive acetazolamide treatment improved hypoxemia at $3100 \mathrm{~m}$ but did not alter performance.

Disclosure: Grants: Swiss National Science Foundation, Lunge Zurich.

\section{P14 \\ Clinical Characteristics of Viral Infections in COPD at Stable State and at Exacerbation}

\author{
D. Stolz ${ }^{1}$, E. Papakonstantinou ${ }^{1}$, L. Grize ${ }^{2}$, D. Schilter ${ }^{3}$, W. Strobel' ${ }^{1}$, \\ R. Louis ${ }^{4}$, C. Schindler ${ }^{2}$, H. Hirsch ${ }^{5}$, M. Tamm ${ }^{1}$ \\ ${ }^{1}$ Clinic of Respiratory Medicine and Pulmonary Cell Research, \\ University Hospital Basel, ${ }^{2}$ Swiss Tropical and Public Health \\ Institute Basel, Basel, ${ }^{3}$ Lindenhof Hospital Bern, Bern, \\ Switzerland, ${ }^{4}$ Department of Pneumology, University of Liege, \\ Liege, Belgium, ${ }^{5}$ Department of Biomedicine, University of \\ Basel, Basel, Switzerland
}

Introduction: Viral respiratory tract infections have been implicated as the predominant risk factor for acute exacerbations of COPD (AECOPD). We aimed to investigate factors associated with viral infections in COPD patients at stable state and at AECOPD.

Methods: We analysed the co-primary end-point of the PREVENT study, an investigator-initiated and driven, controlled trial, that included 450 COPD patients followed for 3 years. Detection of 18 viruses was performed in naso- and oropharyngeal swabs at stable periods $(\mathrm{n}=1909)$, and at AECOPD $(\mathrm{n}=177)$ using a multiplex nucleic acid amplification testing.

Results: Viral infections were identified in 102 cases $(5.3 \%)$ at stable visits and in 68 cases $(38.4 \%)$ at AECOPD. Cough was more common in patients with viral infections $(\mathrm{p}=0.018)$, as also confirmed from CAT score $(\mathrm{p}=0.002)$. Patients with viral infections produced more sputum volume $(\mathrm{p}=0.041)$ and had a higher evidence of potentially pathogenic bacteria in sputum $(\mathrm{p}=0.026)$, particularly for P. Aeruginosa $(\mathrm{p}=0.013)$. In a mixed linear logistic regression model, adjusted for age and sex, an increase of 10 units in SGRQ impact score at stable state was associated with an increased chance of $11 \%$ of having any viral infection (OR: 1.01, 95\% CI 1.00-1.02, $\mathrm{p}=0.032$ ).

Cough and sputum production were more common symptoms in patients having a viral infection at AECOPD $(\mathrm{p}<0.001$ and $p=0.002$, respectively). Edema of the extremities was more frequently present in patients without viral infections $(\mathrm{p}=0.044)$. Patients with viral infection at AECOPD had a higher CAT score $(p=0.007)$ and lower BODE score $(p=0.008)$. In a mixed logistic regression model, adjusted for age and sex, an increase of 1 unit in CAT score was associated with an increased chance of $8.4 \%$ of having any viral infection at AECOPD visit (OR: 1.084, 95\% CI: $1.03-1.14, \mathrm{p}=0.005)$.

Conclusion: Specific factors significantly associated with viral infections may be used as prognostic factors for viral infections in COPD.

Disclosure: Nothing to disclose.

\section{P15 \\ Prevention of Altitude-Related Illness in Patients with COPD by Acetazolamide. Randomized, Placebo-Controlled Trial}

\author{
M. Furian ${ }^{1}$, A. Buergin 1 , P.M. Scheiwiller ${ }^{1}$, L. Mayer ${ }^{1}$, S. Schneider', \\ M. Mademilov'2, B. Emilov' ${ }^{2}$, M. Lichtblau', B. Estebesova², \\ B. Osmonov ${ }^{2}$, L. Muralt ${ }^{1}$, A. Groth' ${ }^{1}$ U. Sheraliev², \\ N.H. Marazhapov' ${ }^{2}$, T.M. Sooronbaev ${ }^{2}$, S. Ulrich ${ }^{1}$, K.E. Bloch ${ }^{1}$ \\ ${ }^{1}$ University Hospital of Zurich, Department of Respiratory \\ Medicine, Sleep Disorders Center, Zurich, Switzerland, \\ ${ }^{2}$ National Center for Cardiology and Internal Medicine, Bishkek, \\ Kyrgyzstan
}

Introduction: In a recent trial, we found that dexamethasone treatment did not prevent altitude-related illness (ARI) in patients with chronic obstructive pulmonary disease (COPD) (Furian, Chest 2018). Therefore, the current randomized, placebo-controlled, double-blind trial evaluated whether acetazolamide prevented ARI in lowlanders with COPD ascending to high altitude.

Methods: $176 \mathrm{COPD}$ patients, $\mathrm{FEV}_{1} \geq 40$ to $<80 \%$ predicted, living below $800 \mathrm{~m}$, were randomized to acetazolamide (125-0$250 \mathrm{mg}$ /day) or placebo starting 24 hours before ascending to and while staying at $3100 \mathrm{~m}$ for 48 hours. Primary outcome was the incidence of any of the following ARI at $3100 \mathrm{~m}$ : acute mountain sickness (AMS, Lake Louise score $>2$ including headache), severe hypoxemia $\left(\mathrm{SpO}_{2}<80 \%,>30 \mathrm{~min}\right)$ or any discomfort requiring medical intervention. Arterial blood gases and other outcomes were also assessed. www.ClinicalTrials.gov NCT03156231.

Results: Of 90 patients (median age 58 y, $\mathrm{FEV}_{1} 64 \%$ predicted) randomized to placebo, 64 (71\%) experienced ARI; of 86 patients (age $58 \mathrm{y}, \mathrm{FEV}_{1} 64 \%$ predicted) randomized to acetazolamide, 35 (41\%) experienced ARI, odds ratio $0.28,95 \%$ CI 0.15 to $0.52, \mathrm{P}<$ 0.001 . Compared to placebo, acetazolamide reduced severe hypoxemia by $77 \%$ (incidence $48 \%$ vs. $12 \%, \mathrm{P}<0.001$ ) without changing AMS incidence ( $35 \%$ vs. $31 \%, \mathrm{P}=0.579)$. At $3100 \mathrm{~m}$, day 2, acetazolamide decreased $\mathrm{PaCO}_{2}$ by $-0.3 \mathrm{kPa}(-0.5$ to -0.1$)$ and increased $\mathrm{PaO}_{2}$ by $0.8 \mathrm{kPa}$ ( 0.5 to 1.1 ) vs. placebo, $\mathrm{P}<0.001$ both instances. No serious adverse events occurred.

Conclusions: In lowlanders with moderate to severe COPD, staying for 48 hours at $3100 \mathrm{~m}$, ARI were common (71\% in placebo group). Acetazolamide reduced the incidence of ARI by $42 \%$ through prevention of severe hypoxemia while the occurrence of AMS remained unchanged. The results suggest that lowlanders with COPD going to high altitude may benefit from preventive treatment with acetazolamide.

Disclosure: Grants: Swiss National Science Foundation, Swiss Lung Foundation, Lunge Zurich. 


\section{P16 \\ Effect of Preventive Acetazolamide Treatment on Sleep-Related Breathing Disturbances in Patients with COPD Staying at High Altitude. Randomized Trial}

M. Furian ${ }^{1}$, L. Tan ${ }^{1}$, A. Buergin ${ }^{1}$, P.M. Scheiwiller ${ }^{1}$, L. Mayer ${ }^{1}$, S. Schneider ${ }^{1}$, M. Mademilov' ${ }^{2}$, B. Emilov' ${ }^{2}$, M. Lichtblau', B. Estebesova ${ }^{2}$, B. Osmonov ${ }^{2}$, L. Muralt ${ }^{1}$, A. Groth ${ }^{1}$, U. Sheraliev ${ }^{2}$, N.H. Marazhapov' ${ }^{2}$, T.M. Sooronbaev ${ }^{2}$, S. Ulrich ${ }^{1}$, K.E. Bloch ${ }^{1}$

${ }^{1}$ University Hospital of Zurich, Department of Respiratory Medicine, Sleep Disorders Center, Zurich, Switzerland, ${ }^{2}$ National Center for Cardiology and Internal Medicine, Bishkek, Kyrgyzstan

Introduction: The current randomized, placebo-controlled, double-blind trial evaluates whether preventive treatment with acetazolamide reduces nocturnal hypoxemia and breathing disturbances in lowlanders with chronic obstructive pulmonary disease (COPD) sleeping at high altitude.

Methods: $176 \mathrm{COPD}$ patients, median age $58 \mathrm{y}, \mathrm{FEV}_{1} 64 \%$ predicted (range $\geq 40$ to $<80 \%$ predicted), living below $800 \mathrm{~m}$, underwent baseline assessments at $760 \mathrm{~m}$ and were randomized to receive acetazolamide (125-0-250 $\mathrm{mg}$ /day) or placebo starting 24 hours before ascending to and while staying at $3100 \mathrm{~m}$ for 2 nights. Co-primary outcomes were mean nocturnal $\mathrm{SpO}_{2}$ and the oxygen desaturation index (ODI, $\mathrm{SpO}_{2}$ dips $>3 \%$ ) recorded during respiratory sleep studies at $3100 \mathrm{~m}$. www.ClinicalTrials.gov NCT03177850.

Results: Over the course of 2 days at $3100 \mathrm{~m}, 10$ patients (12\%) randomized to acetazolamide and 43 patients (48\%) randomized to placebo $(\mathrm{P}<0.001$ vs. acetazolamide $)$ experienced severe nocturnal hypoxemia $\left(\mathrm{SpO}_{2}<80 \%\right.$ for $\left.>30 \mathrm{~min}\right)$ requiring oxygen therapy according to predefined rules. In 69 patients receiving acetazolamide, mean $\pm \mathrm{SD} \mathrm{SpO}_{2}$ at $760 \mathrm{~m}$ and $3100 \mathrm{~m}$, night 1 , were $91 \pm 2 \%$ and $86 \pm 2 \%$; ODI was $6.0 \pm 6.5$ and $13.8 \pm 14.4 / \mathrm{h}$ ( $\mathrm{P}<0.001$, both instances). In 59 patients receiving placebo, $\mathrm{SpO}_{2}$ at $760 \mathrm{~m}$ and $3100 \mathrm{~m}$, night 1 , were $91 \pm 2$ and $84 \pm 2 \%$, ODI $5.9 \pm$ 8.4 and $26.3 \pm 26.6 / \mathrm{h}(\mathrm{P}<0.001$, both instances $)$. Mean differences (95\% confidence interval) in altitude-induced changes with acetazolamide vs. placebo were $\mathrm{SpO}_{2}+2 \%$ ( 1 to $\left.2, \mathrm{P}<0.001\right)$ and ODI $-11.7 / \mathrm{h}(-16.9$ to $-6.5, \mathrm{P}<0.001)$.

Conclusions: In unacclimatized lowlanders with moderate to severe COPD sleeping for 2 nights at $3100 \mathrm{~m}$, preventive acetazolamide treatment reduced the incidence of severe nocturnal hypoxemia and altitude-related periodic breathing.

Disclosure: Grant support: Swiss National Science Foundation, Lunge Zurich.

\section{P17 \\ Association Between Serum Levels of Glycosaminoglycans and Bacterial Infections in Exacerbated and in Stable COPD}

\section{E. Papakonstantinou ${ }^{1,2}$, M. Roth ${ }^{3}$, M. Tamm ${ }^{1}$, D. Stolz}

${ }^{1}$ Clinic of Respiratory Medicine and Pulmonary Cell Research, University Hospital Basel, Basel, Switzerland, ${ }^{2}$ Department of Medicine, Laboratory of Pharmacology, Aristotle University of Thessaloniki, Thessaloniki, Greece, ${ }^{3}$ Department of Biomedicine, University of Basel, Basel, Switzerland

Introduction: Glycosaminoglycans are abundant extracellular matrix (ECM) molecules of the lung that play a key role in airway remodelling in COPD. Bacterial infections in COPD patients may trigger acute exacerbations of COPD affecting the progression of the disease and contributing to morbidity, mortality and hospital admissions. It has been shown that glycosaminoglycans mediate the adherence, colonization and pathogenesis of bacterial infections of the respiratory tract. In the present study, we aimed to investigate the association between the presence of potentially pathogenic bacteria in sputum and serum levels of various glycosaminoglycans at stable COPD and at acute exacerbations of COPD.

Methods: Serum levels of hyaluronic acid (HA), heparan sulphate (HS) and chondroitin sulphate (CS) were measured by ELISA in the serum of 638 COPD patients, included in the PROMISECOPD study, an investigator-driven, multicenter, longitudinal trial, at stable state and at exacerbations (moderate and severe). Potential pathogenic bacteria growth was assessed in the sputum of patients at stable state and at exacerbation.

Results: At stable state, serum levels of CS were significantly higher in patients with positive cultures of potential pathogenic bacteria in sputum $(50.0 \mathrm{pg} / \mathrm{ml}$ [13.0-87.0], median [25-75 percentiles]) compared with patients without growth of potential pathogenic bacteria in sputum $(20.0 \mathrm{pg} / \mathrm{ml}$ [0.0-40.7], median [25-75 percentiles]), $\mathrm{p}=0.035$. However, at moderate and severe exacerbations there were no significant differences in serum levels of CS between patients with and without growth of potential pathogenic bacteria in sputum. Serum levels of HA and HS were not associated with the growth of potential pathogenic bacteria in sputum at stable state and at moderate and severe exacerbations.

Conclusion: The significant association of serum CS with the growth of potential pathogenic bacteria in sputum of COPD patients at stable state indicate that specific glycosaminoglycans may be associated with bacterial infections and subsequent airway remodelling in COPD.

Disclosure: Nothing to disclose. 


\section{P18 \\ Effects of Suboptimal Use of CPAP-Therapy on Symptoms of Obstructive Sleep Apnea: A Randomized, Double-Blind, Controlled Trial}

$\underline{\text { T. Gaisl' }}$, R. Protazy' ', S. Thiel', S.R. Haile' ${ }^{2}$, M. Osswald ${ }^{1}$, M. Roos ${ }^{2}$, K. Bloch', J.R. Stradling ${ }^{3}$, M. Kohler ${ }^{1}$

${ }^{1}$ Department of Pulmonology, UniversitätsSpital Zürich, 2Epidemiology, Biostatistics and Prevention Institute, Universität Zürich, Zurich, Switzerland, ${ }^{3}$ University of Oxford, National Institute for Health Research, Oxford, United Kingdom

Background: High adherence to continuous positive airway pressure (CPAP) represents an effective therapy for obstructive sleep apnea (OSA). However, a large proportion of patients (approx. $40-60 \%$ ) are not optimally adherent. Findings from previous observational studies led to the notion that a mean adherence of more than 4 hours is necessary to effectively treat sleepiness. This is the first interventional trial investigating the effects of suboptimal CPAP-usage on sleepiness parameters in patients with OSA.

Methods: In this 2-week randomized, parallel, double-blind, controlled trial we enrolled moderate-to-severe OSA patients, suboptimally treated with CPAP (3 to 4 hours nightly mean usage over 365 days) from five sleep centers in Switzerland. Patients were allocated by minimization to sham- or therapeutic CPAP. The primary outcome was the change in Epworth Sleepiness Scale (ESS) and the minimal clinically important difference was set at 2.0 points. ClinicalTrials.gov-registration: NCT02781740.

Findings: From May 27, 2016, to November 10, 2018, 57 patients (mean age $60.1 \pm 8.0$ years, $78.9 \%$ men) were randomized. 52 patients $(50 \%$ in each arm) finished the study and were included in the per protocol analysis. After two weeks, the ESS-score increased by +2.9 points $(95 \% \mathrm{CI}+1.3$ to $+4.7 ; \mathrm{p}=0.001)$ in the sham-CPAP group when compared to therapeutic CPAP. A corresponding change of +1.1 points $(95 \% \mathrm{CI}+0.1$ to $+2.4 ; \mathrm{p}=0.044)$ was also observed in the Functional Outcomes of Sleep Questionnaire
(FOSQ). No significant effects on objective sleepiness measures, quality of life (SF-36), or arterial blood pressure were observed.

Conclusion: In a group of suboptimally adherent CPAP-users with moderate-to-severe OSA, CPAP effectively and clinically significantly treats subjective daytime sleepiness. These patients can therefore be encouraged to continue CPAP-therapy when it comes to sleepiness. Whether patients with lower adherence (i.e. $<3$ hours) also benefit from CPAP-therapy remains to be established in future trials.

Disclosure: Nothing to disclose.

\section{P19 \\ Prevalence of Obstructive Sleep Apnea in Patients with Thoracic Aortic Aneurysm: A Prospective, Parallel Cohort Study}

T. Gaisl ${ }^{1}$, P. Baumgartner ${ }^{1}$, P. Rejmer ${ }^{1}$, M. Osswald ${ }^{1}$, M. Roeder ${ }^{1}$, S. Thiel' ${ }^{1}$, S.F. Stämpfli ${ }^{2}$, C.F. Clarenbach ${ }^{1}$, F.C. Tanner ${ }^{3}$, M. Kohler ${ }^{1}$

${ }^{1}$ Klinik für Pneumologie, UniversitätsSpital Zürich, Zürich, ${ }^{2}$ Department für Kardiologie, Luzerner Kantonsspital, Luzern, ${ }^{3}$ Klinik für Kardiologie, UniversitätsSpital Zürich, Zürich,

Switzerland

Background: The pathogenesis and aetiology of thoracic aortic aneurysms (TAA) are largely unknown. Preliminary data from patients with aortic dissection and abdominal aneurysms suggest a causal link of obstructive sleep apnea (OSA) on aortic disease.

Methods: In this prospective parallel-cohort study we 2-to-1matched 208 patients with verified TAA to 104 controls without TAA according to sex, age, height, weight, and left ventricular ejection fraction. All participants underwent an ultrasound of the thoracic aorta and a level-III respiratory polygraphy. OSA was defined as apnoea-hypopnea-index $\geq 5 / h$. The prevalence of OSA was

Table 1. Per protocol analysis on primary and secondary outcomes (for Abstract no P18)

\begin{tabular}{|c|c|c|c|c|c|c|c|}
\hline & \multicolumn{2}{|l|}{ Sham-CPAP } & \multicolumn{2}{|l|}{ Real-CPAP } & \multirow[t]{2}{*}{ Change } & \multirow[t]{2}{*}{$95 \% \mathrm{CI}$} & \multirow[t]{2}{*}{$\mathrm{p}$ value } \\
\hline & Baseline & Follow-Up & Baseline & Follow-Up & & & \\
\hline Epworth Sleepiness Scale, points & $8.2 \pm 5.0$ & $10.4 \pm 5.5$ & $8.7 \pm 3.9$ & $7.8 \pm 3.8$ & +2.9 & +1.3 to +4.7 & 0.001 \\
\hline $\begin{array}{l}\text { Functional Outcomes of Sleep } \\
\text { Questionnaire, points }\end{array}$ & $17.9 \pm 1.9$ & $17.9 \pm 2.0$ & $17.8 \pm 2.9$ & $17.0 \pm 2.0$ & +1.1 & 0.1 to +2.4 & 0.044 \\
\hline $\begin{array}{l}\text { Oxford Sleep Resistance Test, signals } \\
\text { missed }\end{array}$ & $7(4$ to 19$)$ & 17.5 (18 to 33$)$ & $7(2$ to 24$)$ & $6(2$ to 39$)$ & +17 & -15 to +49 & 0.289 \\
\hline $\begin{array}{l}\text { Multiple Unprepared Reaction Time } \\
\text { Test, milliseconds }\end{array}$ & $268 \pm 54$ & $300 \pm 73$ & $312 \pm 87$ & $318 \pm 101$ & +21 & -21 to +65 & 0.317 \\
\hline Systolic blood pressure (office), mm Hg & $132.8 \pm 14.7$ & $132.5 \pm 15.6$ & $133.6 \pm 16.4$ & $129.3 \pm 14.7$ & +2.9 & -3.8 to +9.6 & 0.384 \\
\hline Diastolic blood pressure (office), $\mathrm{mm} \mathrm{Hg}$ & $82.3 \pm 8.3$ & $84.3 \pm 11.6$ & $83.5 \pm 11.2$ & $82.2 \pm 10.6$ & +2.4 & -3.1 to +7.9 & 0.388 \\
\hline Apnea-Hypopnea Index, hr-1 & $4.9(2.5$ to 10.9$)$ & $34.3(23.1$ to 51.7$)$ & $4.1(2.5$ to 7.1$)$ & $3.6(2.2$ to 5.7$)$ & +33.4 & +23.3 to +43.6 & $<0.001$ \\
\hline
\end{tabular}




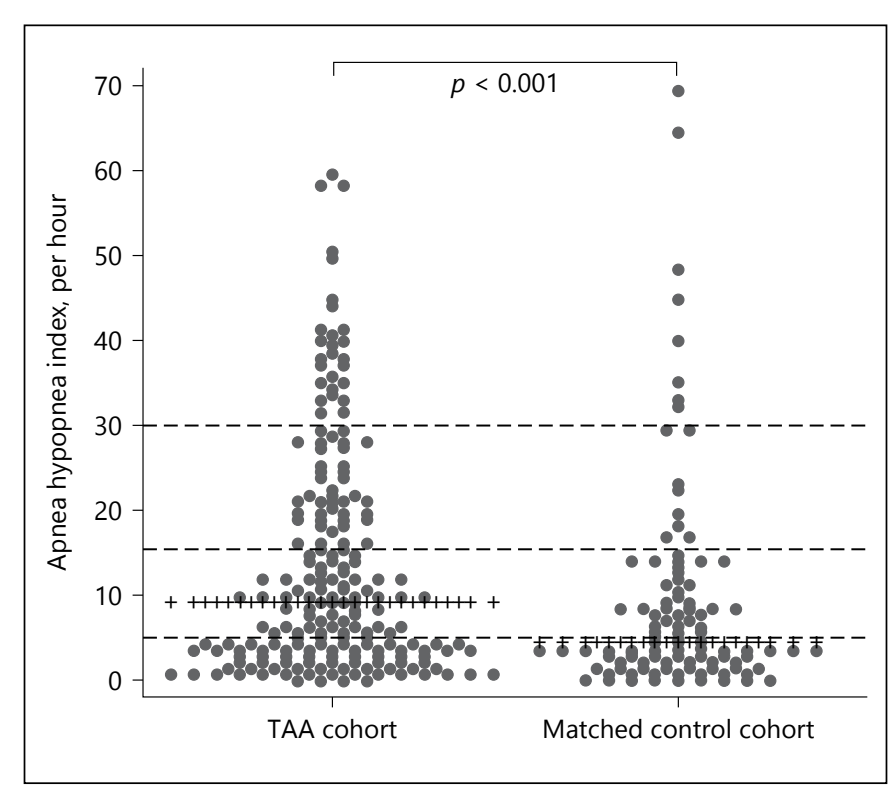

Fig. 1. Apnea-hypopnea index (AHI) by groups (for Abstract no P19).

compared with conditional logistic regression and controlling for the matching variables.

Results: 312 patients (mean age $65 \pm 11$ years, $82 \%$ male, mean body-mass-index $27 \pm 4 \mathrm{~kg} / \mathrm{m}^{2}$ ) were successfully 2-to-1 matched in the final model. Prevalence of OSA was significantly higher in the TAA-group when compared to the matched control group (63\% versus $47 \%$; odds ratio 1.87 [ $95 \%$ CI 1.05 to 3.34 ]; $\mathrm{p}=$ $0.03)$. When applying a higher apnoea-hypopnea index threshold $(\geq 15 / \mathrm{h})$ the odds ratio increased to 3.25 (95\% CI 1.65 to $6.42 ; \mathrm{p}<$ $0.001)$. The median apnea-hypopnea index was higher in patients with TAA (9.2/h [3.3 to 20.0] versus $4.5 / \mathrm{h}$ [2.2 to 11.1 ], $\mathrm{p}<0.001)$.

Conclusion: Patients with TAA have a higher prevalence of OSA when compared to the general population. Since OSA is effectively treatable and might contribute to the pathogenesis of TAA, further longitudinal trials are needed to assess the association between OSA and TAA.

Disclosure: Dr. Gaisl and Prof. Kohler report personal fees from Bayer AG (consultancy), outside the submitted work.

\section{P20 \\ Validation of NoSAS Score for Sleep Apnea Detection in a Clinical Sample}

\author{
S. Herschmann ${ }^{1}$, J. Haba Rubio' ${ }^{1}$, P. Marques-Vidal ${ }^{2}$, R. Heinzer ${ }^{1}$ \\ ${ }^{1} \mathrm{CIRS},{ }^{2} \mathrm{CHUV}$, Lausanne, Switzerland
}

Introduction: The gold standard for sleep apnea detection are sleep recordings (polysomnography or polygraphy). Because of their costs and low availability, they cannot be used as routine exams to detect sleep apnea. Therefore, it is important to have a good screening tool allowing to select patients at higher risk for sleep apnea who need further investigations. The goal of the study is to compare the performances of the 3 most used sleep disordered breathing screening scores in a clinical sample.

Methods: Clinical data and results of sleep exams performed in consecutive adult patients referred to the sleep center of CHUV for a polygraphy or a polysomnography between November 2016 and February 2018 were analysed. The Berlin, STOP-Bang and NoSAS screening scores were calculated and compared with the results of the sleep studies.

Results: Compared with the other screening scores, NoSAS score showed overall the best performances with a negative predictive value of 0.88 , a positive predictive value of 0.43 and a correctly classified rate of $71 \%$. The STOP-Bang had a slightly better negative predictive value $(0.92)$ but a very low positive predictive value (0.30) which yielded a poor correctly classified rate $(47 \%)$. The Berlin score had globally poor results (negative predictive value: 0.85 , positive predictive value: 0.29 and a correctly classified rate: $56 \%)$. Furthermore, NoSAS score had the best area under ROC curves (0.78) compared to STOP bang (0.71) and Berlin (0.62). NoSAS score however yielded a slightly higher rate of false negative than STOP-Bang score. Patients at risk of false negative using NoSAS score are women complaining of snoring with a low neck circumference. Special caution is the warranted in this subpopulation.

Conclusion: NoSAS score seems to show a better discrimination capacity compared to Berlin an STOP Bang scores in a clinical population referred to a sleep center.

Disclosure: Nothing to disclose.

\section{P21 \\ Impact of Hypertension on Cerebral Microvascular Structure in Obstructive Sleep Apnoea Patients: A Diffusion Magnetic-Resonance Imaging Study}

S. Thiel' ${ }^{1}$ T. Gaisl' ${ }^{1}$, F. Lettau' ${ }^{1}$, A. Boss' ${ }^{2}$, S. Winkelhofer ${ }^{3}$, M. Kohler ${ }^{1}$,
C. Rossi

${ }^{1}$ Department of Pulmonology, ${ }^{2}$ Department of Diagnostic and Interventional Radiology, ${ }^{3}$ Department of Neuroradiology, UniversitätsSpital Zürich, Zurich, Switzerland

Introduction: Obstructive sleep apnoea (OSA) is a highly prevalent sleep related breathing disorder associated with hypertension, impaired peripheral vascular function, and an increased risk of stroke. Evidence suggests that abnormalities of the cerebral microcirculation, such as capillary rarefication, may be present in these patients. We evaluated whether the presence of hypertension may affect the cerebral capillary architecture and function assessed by Intravoxel Incoherent Motion (IVIM) magnetic resonance imaging (MRI) in patients with OSA.

Methods: 41 patients ( $88 \%$ male, mean age $57 \pm 10$ years) with moderate-to-severe OSA were selected and divided into two groups (normotensive vs. hypertensive). All hypertensive OSA patients were adherent with their antihypertensive medication. Cerebral microvascular structure was assessed in grey (GM) and white 


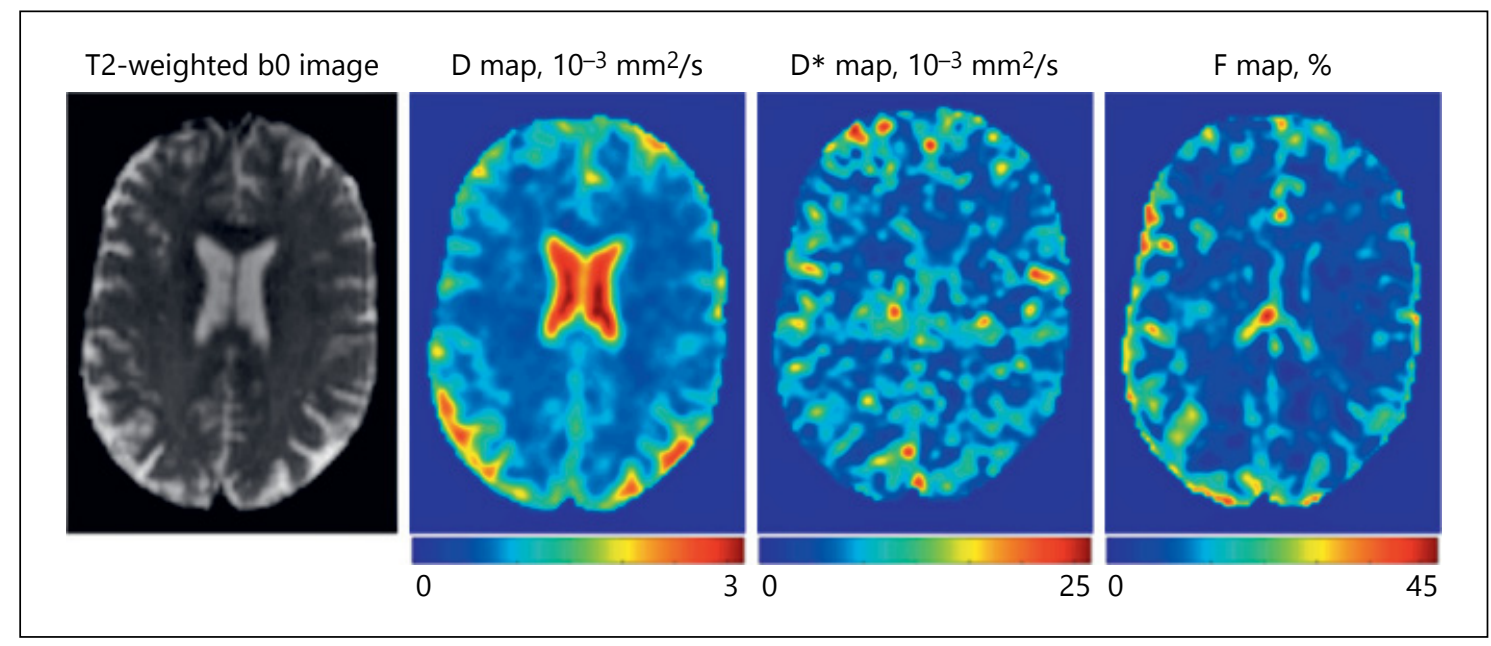

Fig. 1. Representative parametrical maps computed in one normotensive patient (for Abstract no P21).

matter (WM) using an echo-planar diffusion imaging sequence with 14 different b-values. A step-wise IVIM analysis algorithm was applied to compute true diffusion (D), perfusion fraction $(f)$, and pseudo-diffusion $\left(D^{*}\right)$ values. Group comparisons were performed with the Wilcoxon-Mann-Whitney-Test.

Results: Diffusion and perfusion-related indexes in middleaged OSA normotensive patients were quantified in both tissue types $\left(\mathrm{D}\left[10^{-3} \mathrm{~mm}^{2} / \mathrm{s}\right]: \mathrm{GM}=0.83 \pm 0.03 ; \mathrm{WM}=0.72 \pm 0.03 ; \mathrm{D}^{*}\right.$ $\left[10^{-3} \mathrm{~mm}^{2} / \mathrm{s}\right]: \mathrm{GM}=7.72 \pm 0.89 ; \mathrm{WM}=7.38 \pm 0.98 ; f(\%) \mathrm{GM}=$ $0.09 \pm 0.01 ; \mathrm{WM}=0.06 \pm 0.01)$. In the examined tissues types, hypertension did not result in changes on the estimated MRI IVIM index values.

Conclusion: Based on IVIM analysis, cerebral microvascular structure and function showed no difference between hypertensive and normotensive patients with moderate-to severe OSA. Treatment adherence with antihypertensive drug regime and, in turn, controlled hypertension seems not to affect microvascular structure and perfusion of the brain.

Disclosure: Nothing to disclose.

\section{P22 \\ Neurometabolic Changes During CPAP-Withdrawal in Obstructive Sleep Apnoea: Data from Two Randomised Controlled Trials}

\author{
S. Thiel', S.R. Haile ${ }^{2}$, M. Peitzsch ${ }^{3}$, E.I. Schwarz' ${ }^{1}$, N.A. Sievi ${ }^{1}$, \\ F. Beuschlein ${ }^{4}$, M. Kohler ${ }^{1}$, T. Gaisl $^{1}$ \\ ${ }^{1}$ Department of Pulmonology, UniversitätsSpital Zürich, \\ 2Epidemiology, Biostatistics and Prevention Institute, \\ Universität Zürich, Zurich, Switzerland, ${ }^{3}$ Institute of Clinical \\ Chemistry and Laboratory Medicine, University Hospital \\ Carl Gustav Carus, Dresden, Germany, ${ }^{4}$ Department of \\ Endocrinology, Diabetology and Nutrition, UniversitätsSpital \\ Zürich, Zurich, Switzerland
}

Introduction: Obstructive sleep apnoea (OSA) is associated with an increased risk of cardiovascular burden, however the underlying mechanisms are not yet well understood. The aim of this trial was to elucidate the effect of OSA on neurometabolic parameters.

Methods: Data from two 2-week, double-blind, parallel-group, randomised controlled trials (CPAP withdrawal vs CPAP) including 70 patients with OSA (mean age $61 \pm 10$ years, $82 \%$ male) treated with CPAP for more than one year with high compliance were included in the per protocol analysis. Fasting blood samples were taken at baseline in the morning prior to CPAP interruption and following 2 weeks of CPAP withdrawal. Samples were analysed using liquid chromatography tandem mass spectrometry and high pressure liquid chromatography electrochemical detection. A regression analysis adjusting for baseline parameters was performed to determine the treatment effects.

Results: OSA did recur in the CPAP-withdrawal group (mean change apnoea-hypopnea-index +31.8 events/hour, $95 \% \mathrm{CI}+22.8$ to $+40.6, \mathrm{p}<0.001$ ) but remained controlled in the CPAP group. 3, 4-dihydroxyphenylglycol (DHPG), norepinephrine (NE) and cortisol increased after two weeks of CPAP-withdrawal (mean 


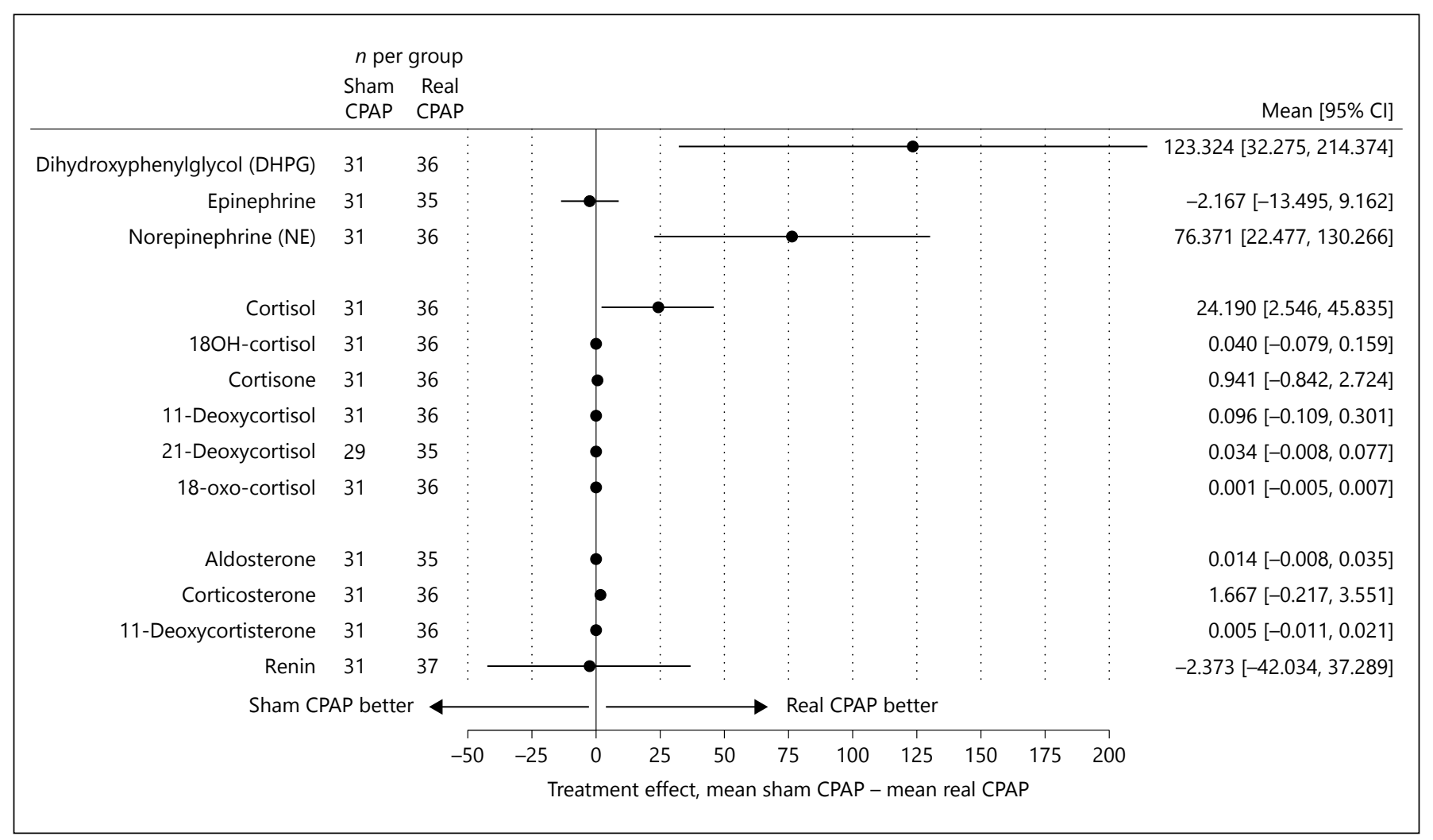

Fig. 1. Treatment effects of the intervention for the primary outcomes (for Abstract no P22).

change DHPG $+123.324 \mathrm{pg} / \mathrm{ml}, 95 \% \mathrm{CI}+32.275$ to $+214.374, \mathrm{p}=$ 0.009 ), (mean change $\mathrm{NE}+76.37 \mathrm{pg} / \mathrm{ml}, 95 \% \mathrm{CI}+22.48$ to +130.27 , $\mathrm{p}=0.006)$, (mean change cortisol $+24.190 \mathrm{ng} / \mathrm{ml}, 95 \% \mathrm{CI}+2.546$ to $+45.835, \mathrm{p}=0.029)$. No statistically significant changes of the renin-angiotensin-aldosterone-system (RAAS) metabolites were documented.

Conclusion: CPAP-withdrawal resulted in elevations of NE, DHPG, and cortisol, whereas no significant changes in the metabolites of the RAAS were documented. Therefore, the activation of the sympathetic nervous system and the hypothalamic-pituitaryadrenal axis might contribute to the blood pressure elevation in patients with OSA.

Disclosure: Nothing to disclose.

\section{Poster Walk 1: Airway and Sleep Disorders}

\section{P23 \\ TNF-a Reduced C/EBP-a Expression by MicroRNA101 Up-Regulation in Airway Smooth Muscle Cells of COPD Patients}

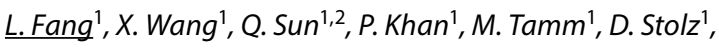 \\ M. Roth ${ }^{1}$ \\ 'Pneumologie, Universitätsspital Basel, Basel, Switzerland, \\ ${ }^{2}$ College of Animal Science and Technology, Northwest A\&F \\ University, Yangling, China
}

Introduction: The molecular mechanisms leading to chronic obstructive pulmonary disease (COPD) are not well understood. TNF- $\alpha$ is amajor contributorto the pathogenesis of COPD by activating airway smooth muscle cells (ASMC), which contribute to chronic inflammation, airway remodeling, and lung deterioration. Deregulated expression of the transcription factor C/EBP- $a$ caused ASMC remodeling in other chronic lung diseasessuch as 
asthma. C/EBP- $\alpha$ has a highly conserved binding site for miR101. The latter was increased in COPD lungs by cigarette smoking. This study investigated the regulatory effect of miR101 on C/ EBP- $\alpha$ expression and its role in the activation of ASMC from COPD patients.

Methods: Human ASMC were isolated from bronchial endoscopic biopsies of COPD patients $(n=5)$ and healthy donors $(n=$ 5). Cells were stimulated with TNF- $\alpha(10 \mathrm{ng} / \mathrm{ml})$ over various periods, and miR101 expression was detected by real-time PCR. The regulatory effect of miR101 was investigated by in vitrotransfection of ASMC with miR101 mimic or anti-sense miR101. Western blotting was used for protein expression of collagen type-I, COX-2, C/EBP- $\alpha$, and C/EBP- $\beta$.

Results: The base-line expression of miR101 was significant higher in ASMC from COPD patients compared to healthy control ASMC $(\mathrm{P}<0.05)$. In ASMC from COPD patients, TNF-afurther up-regulated miR101 expression significantly at 3, 6, and 24 hours. In contrast,TNF- $\alpha$ significantly decreased $\mathrm{C} / \mathrm{EBP}-\alpha$ expression $(\mathrm{P}<0.05)$ in ASMC from COPD patiens, while increased C/EBP- $\beta$ expression $(\mathrm{P}<0.05)$. In addition, increased collagen-type-I synthesis and imbalanced mitochondrial protein COX-2 production were induced $(p<0.05)$. Overexpression or knockdown of miR101 in ASMC confirmed its regulatory effect of on C/EBP- $\alpha$ expression. Similar effect were observed in control cells.

Conclusion: TNF-aincreased the expression of miR101, which reduced $\mathrm{C} / \mathrm{EBP}-\alpha$ expression and modified mitochondrial activity in pro-inflammatory ASMC. Thus, miR101 may represent a novel target for chronic airway remodeling in COPD therapy.

Disclosure: Nothing to disclose.

\section{P24 \\ Serum Glycosaminoglycans are Differentially Associated with Collagen Synthesis and Degradation in COPD}

\section{E. Papakonstantinou ${ }^{1,2}$, M. Roth ${ }^{3}$, M. Tamm 1 , D. Stolz 1}

${ }^{1}$ Clinic of Respiratory Medicine and Pulmonary Cell Research, University Hospital Basel, Basel, Switzerland, ${ }^{2}$ Department of Medicine, Laboratory of Pharmacology, Aristotle University of Thessaloniki, Thessaloniki, Greece, ${ }^{3}$ Department of Biomedicine, University of Basel, Basel, Switzerland

Introduction: Glycosaminoglycans and collagens are the most abundant extracellular matrix (ECM) molecules that play a significant role in the structure and function of the lung. In the present study, we aimed to investigate the association between serum glycosaminoglycans and collagen synthesis and degradation in COPD.

Methods: The glycosaminoglycans hyaluronic acid (HA), heparan sulphate (HS) and chondroitin sulphate (CS) were measured by ELISA in the serum of 638 COPD patients included in the PROMISE-COPD study, an investigator-driven, multicenter, longitudinal trial, performed at 11 tertiary respiratory centers in 8 European countries. Pro-forms of collagens type III, type V and type VI, degradation fragments of collagens type I, type IV ( $a 1$ chain and $\alpha 3$ chain), type VI, and neutrophil elastase were measured with Nordic Bioscience assays.

Results: HA and HS were significantly associated with proforms of collagens III and VI ( $p<0.001$ for all), the major collagens found in the lung interstitium and in large bronchi. CS was not associated with any of the pro-forms or degradation products of collagens tested. Furthermore, there was no association of HA, HS and CS with neutrophil elastase. None of the glycosaminoglycans tested was associated with diffusion capacity or with quantitative CT scan parameters representing the status of lung parenchyma, indicating that HA, HS and CS are not related to emphysema. However, glycosaminoglycans were associated with markers of inflammation, as HA was found to associate with adrenomedullin $(\mathrm{p}=0.001)$ and with atrial natriuretic peptide (ANP) $(\mathrm{p}<0.001)$ and HS was found to associate with procalcitonin $(\mathrm{p}=$ $0.001)$, adrenomedullin $(\mathrm{p}<0.001)$, copeptin $(\mathrm{p}<0.001)$ and ANP $(\mathrm{p}<0.001)$.

Conclusion: The significant association of specific glycosaminoglycans with collagen pro-forms and with COPD prognostic markers indicate that these molecules are part of ECM turnover in COPD and may be involved in airway remodelling in this disease.

Disclosure: Nothing to disclose.
P25
Effect of Preventive Acetazolamide Treatment on Visuo-Motor Learning Performance in Lowlanders with COPD Staying at High Altitude. Randomized Trial
P.M. Scheiwiller ${ }^{1}$, M. Furian ${ }^{1}$, A. Buergin ${ }^{1}$, C. Meier ${ }^{1}$, L. Mayer ${ }^{1}$, S. Schneider ${ }^{1}$, M. Mademilov'2, B. Emilov'2, M. Lichtblau', B. Estebesova ${ }^{2}$, B. Osmonov ${ }^{2}$, L. Muralt ${ }^{1}$, A. Groth ${ }^{1}$, U. Sheraliev ${ }^{2}$, N.H. Marazhapov' ${ }^{2}$, T.M. Sooronbaev ${ }^{2}$, S. Ulrich ${ }^{1}$, K.E. Bloch ${ }^{1}$
${ }^{1}$ University Hospital of Zurich, Department of Respiratory Medicine, Sleep Disorders Center, Zurich, Switzerland,
${ }^{2}$ National Center for Cardiology and Internal Medicine, Bishkek, Kyrgyzstan

Introduction: Hypoxia may impair visuo-motor learning of healthy individuals (Stadelmann, Physiol. Behav. 2015). The current randomized, placebo-controlled, double-blind trial evaluated whether such impairments occurred in lowlanders with chronic obstructive pulmonary disease (COPD) staying at high altitude and whether this can be prevented by acetazolamide treatment.

Methods: 46 patients with $\mathrm{COPD}, \mathrm{FEV}_{1} \geq 40$ to $<80 \%$ predicted, living $<800 \mathrm{~m}$, were randomized to acetazolamide $(\mathrm{N}=23,125-0$ $250 \mathrm{mg}$ /day) or placebo $(\mathrm{N}=23)$ starting $24 \mathrm{~h}$ before ascending to and while staying at $3100 \mathrm{~m}$ for $48 \mathrm{~h}$. During $30 \mathrm{~min}$ Motor Task Manager tests (MTMT) patients had to operate a mouse in order to let a pointer hit moving targets displayed repeatedly at variable positions on a computer screen. After evening practice sessions, the immediate (within $10 \mathrm{~min}$ ) and delayed performance (in the morning after overnight sleep) were quantified by the directional error of pointer vs. target trajectories as indices of visuo-motor memory retrieval. www.ClinicalTrials.gov NCT03165890. 
Results: Multivariable regression analyses of data from patients using placebo revealed an increase in mean directional error during immediate and post-sleep MTMT at $3100 \mathrm{~m}$ vs. $760 \mathrm{~m}$ of $6^{\circ}$ (95\% confidence interval 5 to 7), both instances. Acetazolamide improved directional errors in immediate MTMT by $3^{\circ}$ (95\% CI 2 to 4 ) and post-sleep MTMT by $4^{\circ}$ (95\% CI 2 to 5$), \mathrm{P}<0.001$, both changes.

Conclusions: During sojourns at $3100 \mathrm{~m}$, lowlanders with moderate to severe COPD experienced impairments in immediate and post-sleep MTMT performance that were significantly mitigated by acetazolamide. The results suggest beneficial effects of preventive acetazolamide treatment on visuo-motor learning performance in COPD patients exposed to hypobaric hypoxia.

Disclosure: Grants: Swiss National Science Foundation, Swiss Lung Foundation, Lunge Zurich.

\section{P26 \\ Altitude-Induced Impairment in Postural Control in Patients with COPD. Randomized, Placebo-Controlled Trial of Acetazolamide}

\author{
A. Buergin' ${ }^{1}$, M. Furian ${ }^{1}$, P.M. Scheiwiller ${ }^{1}$, L. Mayer ${ }^{1}$, S. Schneider', \\ M. Mademilov' ${ }^{2}$ B. Emilov', M. Lichtblau', B. Estebesova², \\ B. Osmonov ${ }^{2}$, L. Muralt ${ }^{1}$, A. Groth ${ }^{1}$, U. Sheraliev ${ }^{2}$, \\ N.H. Marazhapov ${ }^{2}$, T.M. Sooronbaev ${ }^{2}$, S. Ulrich ${ }^{1}$, K.E. Bloch ${ }^{1}$
}

${ }^{1}$ University Hospital of Zurich, Department of Respiratory Medicine, Sleep Disorders Center, Zurich, Switzerland,

${ }^{2}$ National Center for Cardiology and Internal Medicine, Bishkek, Kyrgyzstan

Introduction: Postural control $(\mathrm{PC})$ in patients with chronic obstructive pulmonary disease (COPD) is impaired at sea level and further deteriorated during exposure to hypoxia at altitude $(\mathrm{Mu}-$ ralt, Front. Physiol. 2018). This randomized, placebo-controlled, double-blind trial evaluates the effectiveness of acetazolamide in preventing PC-impairment in lowlanders with COPD travelling to $3100 \mathrm{~m}$.

Methods: $127 \mathrm{COPD}$ patients, $\mathrm{FEV}_{1} \geq 40 \%$ to $<80 \%$ predicted, living $<800 \mathrm{~m}$, were randomized to receive acetazolamide (125$0-250 \mathrm{mg}$ /day) or placebo starting $24 \mathrm{~h}$ before and during $48 \mathrm{~h}$ at $3100 \mathrm{~m}$. Pulse oximetry and PC were assessed at $760 \mathrm{~m}$ and one day after arrival at $3100 \mathrm{~m}$ by letting patients stand on a Wii Balance Board ${ }^{\mathrm{TM}}$, recording the center of pressure path length (PL, the primary outcome) during 5 trials of $30 \mathrm{sec}$ each. www.ClinicalTrials. gov NCT03177811.

Results: With ascent from $760 \mathrm{~m}$ to $3100 \mathrm{~m}$, patients receiving placebo had a mean \pm SD PL increase from $28.78 \pm 9.68$ to $30.04 \pm$ $9.95 \mathrm{~cm}(\mathrm{P}=0.002)$. In patients receiving acetazolamide the change from $27.64 \pm 9.61$ to $28.38 \pm 9.65 \mathrm{~cm}$ was nonsignificant $(\mathrm{P}=$ $0.069)$. The mean between-group difference in altitude-induced PL change (treatment effect) was $-0.54 \mathrm{~cm}$ (95\% CI -1.66 to 0.58 , $\mathrm{P}=0.289$ ). Regression analysis controlling for several confounders confirmed an increase in PL at altitude $(+0.98 \mathrm{~cm}, 95 \%$ CI 0.39 to $1.58, \mathrm{P}=0.001)$ but no significant effect of acetazolamide $(0.66 \mathrm{~cm}$,
$95 \% \mathrm{CI}-0.25$ to $1.57, \mathrm{P}=0.156)$. PL changes occurred mostly in the anteroposterior (AP) plane; AP sway amplitude increased by $0.3 \mathrm{~cm}(95 \%$ CI 0.15 to 0.45$)$ with acetazolamide $(\mathrm{P}<0.001)$. Pulse oximetry at 760 and $3100 \mathrm{~m}$ with placebo was $95 \pm 2 \%, 88 \pm 3 \%$, with acetazolamide $94 \pm 2 \%, 89 \pm 3 \%$ ( $\mathrm{P}<0.001$ both changes), the effect of acetazolamide was $+2 \%$ (95\% CI 1 to 3 ).

Conclusions: In lowlanders with COPD postural control was impaired during a stay at $3100 \mathrm{~m}$ and this was not prevented by acetazolamide although the drug improved arterial oxygen saturation.

Disclosure: Grant: Swiss National Science Foundation, Swiss Lung Foundation, Lunge Zurich.

\section{P27 \\ The Course of Handgrip Strength Is No Indicator for Mortality in COPD: A Longitudinal Cohort Study}

\author{
D. Kohlbrenner ${ }^{1}$, N.A. Sievi ${ }^{1}$, R. Thurnheer ${ }^{2}$, J.D. Leuppi ${ }^{3}$, S. Irani ${ }^{4}$, \\ M. Frey ${ }^{5}$, M. Brutsche ${ }^{6}$, T. Brack ${ }^{7}$, M. Kohler ${ }^{1}$, C.F. Clarenbach ${ }^{1}$ \\ ${ }^{1}$ Department of Pulmonology, University Hospital \\ Zurich, Zurich, ${ }^{2}$ Pulmonary Division, Cantonal Hospital of \\ Münsterlingen, Münsterlingen, ${ }^{3}$ University Clinic of Medicine, \\ Cantonal Hospital Baselland and University of Basel, Liestal, \\ ${ }^{4}$ Pulmonary Division, Cantonal Hospital of Aarau, Aarau, \\ ${ }^{5}$ Pulmonary Division, Clinic Barmelweid, Barmelweid, \\ ${ }^{6}$ Pulmonary Division, Cantonal Hospital of St. Gallen, St. Gallen, \\ ${ }^{7}$ Pulmonary Division, Cantonal Hospital of Glarus, Glarus, \\ Switzerland
}

Introduction: The assessment of handgrip strength (HGS), a measure of maximal force, is easily performed and of prognostic value in chronic conditions. In a cross-sectional setting, HGS is associated with mortality and health-related quality of life in COPD. However, no data are published investigating the longitudinal course of HGS and its possible predictors.

Methods: A longitudinal cohort study with annual assessment of various disease and physical activity (PA) markers was performed in COPD patients (GOLD stages II-IV). HGS was assessed by handgrip dynamometry; PA was assessed using a triaxial accelerometer. Univariate and multivariate mixed effect regression models were used to compute the influence of possible confounders on the annual changes in HGS.

Results: 194 COPD patients with a median (quartiles) followup time of 2.1 (1.54/3.01) years were assessed. HGS of the dominant side significantly decreased over time (Coef. (95\% CI) of -0.86 $(-1.09 /-0.62), \mathrm{p}<0.001)$, and was associated with the decrease in baseline-adjusted predicted values of $\mathrm{FEV}_{1}$ (Coef. (95\% CI) of 0.14 $(0.003 / 0.03), \mathrm{p}=0.016)$ and change in baseline-adjusted predicted FEV $_{1}$ (Coef. $(95 \% \mathrm{CI})$ of $\left.-0.04(-0.08 /-0.01), \mathrm{p}<0.021\right)$. Patients who died showed no greater decline over time in HGS adjusted for baseline than patients who survived (Coef. (95\% CI) of -0.80 $(-1.75 / 0.16), \mathrm{p}=0.101)$ (see Figure 1).

Conclusion: HGS significantly decreases over time with an increased airway obstruction as a risk factor for an accelerated annual decline. Patients who died did not show a greater decline in 


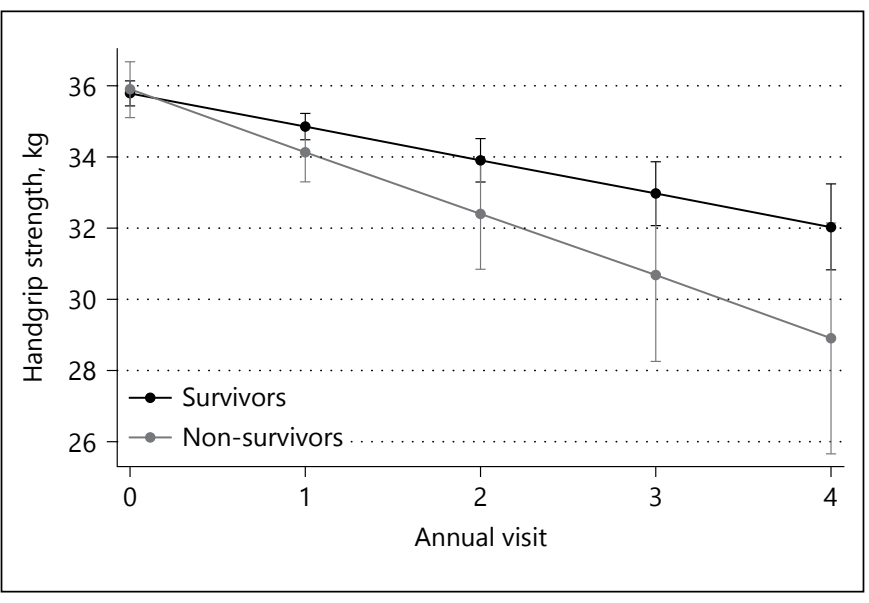

Fig. 1. Annual decline of handgrip strength (for Abstract no P27).

HGS than patients who survived. The course of HGS was not an indicator for mortality up to seven years.

Disclosure: Nothing to disclose.

P28

Swiss Severe Asthma Register (SAR)

C. Gregoriano' ${ }^{1}$ A. Azzola ${ }^{2}$, P.-O. Bridevaux ${ }^{3,4}$, F. Charbonnier ${ }^{5}$, C. Clarenbach ${ }^{6}$, H.-W. Duchna ${ }^{7}$, L. Kern ${ }^{8}$, T. Rothe ${ }^{9}$, C. von Garnier ${ }^{10}$, V. Mangold ${ }^{11}$, J.D. Leuppi ${ }^{1,12}$

${ }^{1}$ University Clinic of Medicine, Cantonal Hospital Baselland, Liestal, ${ }^{2}$ Pneumologia e Medicina Intensiva, Ospedale Civico, Lugano, ${ }^{3}$ Service de Pneumologie, Hôpital du Valais, Sion, ${ }^{4}$ Pneumology, University Hospital of Geneva, University of Geneva, ${ }^{5}$ Hôpitaux Universitaires Genève, Geneva, ${ }^{6}$ Pulmonary Division, University Hospital Zurich, Zurich, ${ }^{7}$ Hochgebirgsklinik Davos, Davos, ${ }^{8}$ Department of Pulmonary Medicine, Cantonal Hospital St. Gallen, St. Gallen, ${ }^{9}$ Pneumology, Cantonal Hospital Graubünden, Chur, ${ }^{10}$ Respiratory Medicine Department, Inselspital, University of Bern, Bern, ${ }^{11}$ Kantonsspital Baselland, ${ }^{12}$ Medical Faculty, University of Basel, Basel, Switzerland

Background: The treatment of severe asthma has been revolutionized by the introduction of targeted therapies since 2005 . Nowadays, many patients with a severe asthma diagnosis are treated with such medications. However, the long-term effects of this specific therapies are only partially known and many unanswered questions remain. The treatment and care situation of severe asthma in Switzerland is insufficient as in many other countries. Therefore, the data of a register offers a fascinating opportunity describe the day-to-day care of these patients in a descriptive way and thus making the treatment and care transparent and optimizing it.

Objective: The overall objective of this study is to establish a clinical register for patients with severe asthma. The primary objective is to show changes in symptoms control during follow up period and at study end by using the Asthma Control Test. Secondary endpoints are to collect data to better understand asthma's natural history in patients with severe asthma.

Methods: For this multicenter longitudinal prospective cohort study, patients with a severe asthma will be recruited and enrolled by local pulmonologist in private practice and pulmonary departments in hospitals within Switzerland. The register will collect socio-demographic information as well as medical data stored in a non-public register. Four months after inclusion patients should be re-evaluated. Thereafter, there will be an annual follow-up for 15 years. The Swiss Register is based on the already existing "German Register of Severe Asthma”.

Results: Recruitment will start in January 2019. It is expected that approximately 276 patients will be included in the first year.

Discussion: The data of this register will help to improve the medical care of patients with severe asthma and to educate and inform the entire population about this disease.

Disclosure: Nothing to disclose.

\section{P29 \\ Improvement in Self-Reported Physical Functioning with Tiotropium/Olodaterol in a Subgroup of Swiss COPD Patients Participating in a Large International Trial}

\begin{abstract}
M. Tamm ${ }^{1}$, D.M. Schumann ${ }^{1}$, J. Diedrich ${ }^{2}$, J. Kocianova ${ }^{3}$, V. Bayer Zubek ${ }^{4}$, A. Medvedchikov ${ }^{5}$, M. Haaksma-Herczegh ${ }^{5}$, J. Mucsci ${ }^{6}$, Z. Fridlender ${ }^{7}$, C. Toma ${ }^{8}$, A. Belevsky ${ }^{9}$, B. Matula ${ }^{10}$, J. Sorli ${ }^{11}$, A. Valipour ${ }^{12}$
\end{abstract}

${ }^{1}$ Clinic of Pneumology and Pulmonary Cell Research, University Hospital Basel, ${ }^{2}$ Boehringer Ingelheim Pharmaceuticals, Inc, Basel, Switzerland, ${ }^{3}$ MephaCentrum a.s, Ostrava-Poruba, Czech Republic, ${ }^{4}$ Boehringer Ingelheim Pharmaceuticals, Inc, Ridgefield, CT, United States, ${ }^{5}$ Boehringer Ingelheim Pharmaceuticals, Inc, Vienna, Austria, ${ }^{6}$ Elizabeth Nursing Home, Gödöllo, Hungary, ${ }^{7}$ Hebrew University Hadassah Medical School, Jerusalem, Israel, ${ }^{8}$ Institute of Pneumatology 'Marius Nasta', Bucharest, Romania, ${ }^{9}$ Pletney Hospital, Pirogov Russian National Research Medical University, Moscow, Russian Federation, ${ }^{10}$ Specialized Hospital of Saint Zoerardus, Nitra, Slovakia, ${ }^{11}$ Topolsica Hospital, Topolsica, Slovenia, ${ }^{12}$ Ludwig Boltzmann Institute for COPD and Respiratory Epidemiology, Otto Wagner Hospital, Vienna, Austria

Introduction: Reduced physical activity is associated with increased morbidity and mortality in patients with COPD. This study assessed the effects of a fixed-dose tiotropium/olodaterol combination (delivered via Respimat ${ }^{\circledR}$ ) on physical functioning in patients with stable COPD in a 'real-world setting'.

Methods: An open-label single-arm study evaluating therapeutic success, defined as a minimum 10-point increase in the 10-question Physical Functioning Questionnaire (PF-10) score, patient general condition (measured by Physician's Global Evaluation (PGE) score) and patient satisfaction with the Respimat ${ }^{\circledR}$ 
device (rather satisfied, satisfied or very satisfied). Patients were recruited from GP offices.

Results: 109/7218 COPD patients were included in Switzerland. GOLD stages were $22.5 \%$ A, $42.9 \%$ B, 7.1\% C, 27.6\% D. Comorbidities were: cardiac $67 \%$, metabolic $45 \%$, gastrointestinal $24 \%$, psychiatric $21 \%$ and vascular $18 \% .40 \%$ of the patients were smokers at study entry.

A 10-point increase in the PF-10 score after 6 weeks of treatment was observed in $62.8 \%$ [ $95 \%$ CI $52.2-72.5 \%$ ] of patients. The median PF-10 score was 55 at baseline and 65 at Visit 2 . The general condition of the patients at baseline measured by PGE score was predominantly 4 (33.0\% of the patients) or 6 (21.3\% of patients) and at visit 2 mostly 5 (28.7\% of patients) or 6 (26.6\% of patients). The patients were 'rather satisfied' with Spiolto ${ }^{\circledR}$ Respimat ${ }^{\circledR}$ treatment $(89.4 \%)$, with inhaling from the Respimat ${ }^{\circledR}$ device $(94.7 \%)$ and with the handling of the device $(88.3 \%)$. No patients died during the study. Two patients (dizziness; epistaxis) showed at least one investigator-defined drug-related adverse event (AE) and no patients had a serious AE.

Conclusion: More than half the Swiss COPD patients experienced a clinically significant improvement in physical functioning with dual bronchodilation and were 'rather satisfied' with the handling of the Respimat. Although the majority of patients suffered from comorbidities, only two mild, possibly drug-related adverse events occurred. heim.

Disclosure: AV resports personal fees from Boehringer Ingel-

\section{P30 \\ Multi-Walled Carbon Nanotubes Activate and Shift Polarization of Pulmonary Macrophages and Dendritic Cells in an in vivo Model of COPD}

\author{
S. Beyeler ${ }^{1}$, C. Wotzkow ${ }^{2}$, S. Steiner ${ }^{2}$, A. Adhanom Sengal ${ }^{1}$, P. Wick ${ }^{3}$, \\ S. Tschanz ${ }^{4}$, B. Hänni ${ }^{4}$, C. von Garnier ${ }^{1}$, F. Blank $^{1}$ \\ ${ }^{1}$ Pulmonary Medicine, Department of BioMedical Research, \\ University Hospital Bern (Inselspital), ${ }^{2}$ Department of \\ BioMedical Research, University of Bern, Bern, ${ }^{3}$ Empa Materials, \\ Science and Technology, St. Gallen, ${ }^{4}$ Institute of Anatomy, \\ University of Bern, Bern, Switzerland
}

Introduction: With progress of nanotechnology there is considerable concern about adverse health-effects of inhaled occupational nanoparticles, such as multi-walled carbon nanotubes (MWCNT), particularly in the respiratory tract in persons suffering from chronic obstructive pulmonary disease (COPD).

Methods: We utilized an Elastase/LPS COPD mouse model in which healthy controls and COPD animals received $0.08 \mu \mathrm{g} / \mathrm{cm}^{2}$ of MWCNT by intratracheal instillation. Lung function, morphometry, differential counts of the bronchoalveolar lavage fluid, as well as phenotype and activation of alveolar macrophages (AM) and dendritic cells (DC) were monitored in the respiratory tract by flow cytometry.

Results: Treatment with MWCNT caused accumulation of AM in bronchoalveolar lavage fluid (BALF) from COPD groups that increased from $24 \mathrm{~h}$ to $7 \mathrm{~d}$. In the COPD group, MWCNT treatment shifted AM polarization towards a M1-like predominant phenotype, as measured by the ratio of the markers CD38 to CD206, and increased AM activation with upregulation of costimulatory markers CD40 and CD80. Moreover, MWCNT treatment increased the frequencies of DC, leading to an expansion of the $\mathrm{CD} 11 \mathrm{~b}^{+} \mathrm{CD} 103^{-} \mathrm{DC}$ subset. Though MWCNT did not trigger lung functional or structural changes, they induced an increased expression of the muc5AC transcript in COPD animals.

Conclusion: Our data provides initial evidence that inhaled MWCNT affect the pulmonary mucosal immune system by altering both phenotype and activation of antigen-presenting cell populations. Extrapolating these in vivo findings to human pulmonary exposure, caution is warranted when handling inhalable nanofibers.

Disclosure: Nothing to disclose.

\section{P31 \\ Early Spirometry After Bronchoscopic Lung Volume Reduction with Endobronchial Valves}

\section{P. Bezel, G. Straub, D. Franzen}

Interventionelles Lungenzentrum, University Hospital Zurich, Zürich, Switzerland

Background: Bronchoscopic lung volume reduction (BLVR) using endobronchial valves (EBV) has been shown to improve quality of life in patients with severe emphysema and hyperinflation while holding an overall low complication rate. Pneumothorax (PTX) is the most common complication, occurring mostly within the first 2 days. However, 15\% of PTX are observed after hospital discharge. We hypothesized that an early spirometry within 3 days after BLVR could anticipate late-PTX by monitored provocation during spirometry maneuver. Furthermore, we aimed to evaluate early spirometry as predictor of a successful treatment.

Methods: Patients undergoing BLVR at the University Hospital Zurich $(\mathrm{n}=35)$ between 01.11.2017 and 30.11.2018 were included in this retrospective study. The primary endpoint was the occurrence of PTX during and after early spirometry with a follow-up time of 3 months. The secondary endpoint was postinterventional increase in forced expiratory volume in 1 second (FEV1) in early spirometry as well as after 1 month.

Results: 7 patients had PTX before early spirometry leading to exclusion from the study. There were no cases of PTX during or immediately after early spirometry and no cases of late-PTX. In 20 patients completing the follow-up, there was no significant difference in FEV1 between early spirometry and after 1 month ( $p=$ $0.4)$. However, an increase of FEV1 above the minimal clinically important difference (MCID) of $15 \%$ was seen in 8 patients in early spirometry and in 14 patients after 1 month, showing a negative predictive value for early spirometry of $50 \%$ while holding a positive predictive value of $89 \%$.

Conclusion: Early spirometry as a means of provocation did not trigger PTX proving it a safe procedure and possibly anticipates late-PTX. Increase of FEV1 in early spirometry proves successful BLVR, challenging the need for further spirometric follow- 
up. However, negative results don't rule out increase of FEV1 at a later time.

Disclosure: Nothing to disclose.

\section{P32 \\ Efficacy of Pharmacotherapy for Obstructive Sleep Apnea in Adults: A Systematic Review and Network Meta-Analysis}

T. Gaisl' ', S.R. Haile' ${ }^{2}$ S. Thiel' ${ }^{1}$ M. Osswald ${ }^{1}$, M. Kohler ${ }^{1}$

${ }^{1}$ Klinik für Pneumologie, UniversitätsSpital Zürich, Zürich,

${ }^{2}$ Epidemiology, Biostatistics and Prevention Institute,

Universität Zürich, Zurich, Switzerland

Rationale and Objectives: Obstructive sleep apnea (OSA) is highly prevalent and has significant health implications but treatment options are limited. The purpose of this systematic review and network meta-analysis is to summarize evidence on the efficacy of pharmacotherapy in adults with OSA and delineate the underlying mechanisms.

Methods: Seven Databases (Medline, EMBASE, Cochrane, Web of Science, PsycInfo, Clinicaltrials.gov, ICTRP-WHO) were systematically screened for randomized controlled trials (RCTs) from their inception to September 01, 2018. According to a preregistered study protocol (PROSPERO-ID-CRD42018086446) network meta-analysis was performed to obtain intervention effects on the apnea-hypopnea-index (AHI) based on data extracted from published reports.

Main Results: We identified 58 RCTs ( $\mathrm{n}=1,710$ patients) investigating 44 different drugs or drug-combinations. Interventions were classified into seven pathomechanism-groups and summarized narratively. A meta-analysis of 17 trials for seven drugs versus placebo (acetazolamide, donepezil, mirtazapine, ondansetron, paroxetine, protriptyline, theophylline) indicated a small effect for acetazolamide (mean difference in AHI $-9.6 / \mathrm{h}$ $[-17.7,-1.4] ; \mathrm{p}=0.02)$. In the network meta-analysis $\left(\mathrm{I}^{2}=50 \%\right)$ nine drugs (tramazoline, liraglutide, spironolactone/furosemide, acetazolamide, dronabinol, zonisamide, phentermine, spironolactone, and ondansetron/fluoxetine) significantly lowered the AHI compared to placebo.

Conclusions: Although some trials indicate favorable outcomes, these results are only valid for distinctive OSA-phenotypes or were not clinically significant. The effect sizes were small, the majority of trials were not adequately powered. There is currently insufficient evidence to recommend any pharmacotherapy for OSA and no phase-III trials are available. Future studies are advised to account for the underlying mechanisms of the intervention, as current evidence suggests that pharmacotherapy may not be a stand-alone therapy in OSA.

Disclosure: TG and MK served as consultants for Bayer AG for the conduction of a phase-II trial (BAY2253651) in pharmacotherapy in OSA. All other authors declare no competing interests.

\section{P33 \\ Effects of CPAP Treatment on Sex Hormone Levels in Obstructive Sleep Apnoea Patients - Data from Two Randomised, Controlled Trials}

S. Thiel' ${ }^{1}$, F. Grewe ${ }^{1}$, S.R. Haile ${ }^{2}$, M. Peitzsch ${ }^{3}$, E.I. Schwarz', N.A. Sievi ${ }^{1}$, F. Beuschlein ${ }^{4}$, M. Kohler ${ }^{1}$, T. Gaisl $^{1}$

'Department of Pulmonology and Sleep Disorders Centre, UniversitätsSpital Zürich, ${ }^{2}$ Epidemiology, Biostatistics, and Prevention Institute, Universität Zürich, Zürich, Switzerland, ${ }^{3}$ Universität Dresden, Dresden, Germany, ${ }^{4}$ Department of Endocrinology, Diabetology and Nutrition, UniversitätsSpital Zürich, Zürich, Switzerland

Introduction: Erectile dysfunction (ED) and sexual dysfunction (SD) are often concomitantly present in patients with obstructive sleep apnoea (OSA). Treatment with continuous positive airway pressure (CPAP) improves both ED and SD. However, the underlying metabolic mechanisms including the role of sex hormones are not yet fully understood. The aim of this trial was to evaluate the effect of CPAP withdrawal on sex hormone levels in patients with OSA.

Methods: Fasting blood samples of 70 patients (mean age $61 \pm$ 10 years, $82 \%$ male) from two 2 -week, double-blind, parallelgroup, randomised controlled trials (CPAP withdrawal vs. CPAP) were included in the per protocol analysis. The investigated sex hormones included androstenedione, dehydroepiandrosterone (DHEA), 17-hydroxyprogesterone, progesterone, testosterone, pregnenolone, follicle-stimulating hormone, and luteinizing hormone. A regression analysis adjusting for baseline variables and sex was performed to determine the treatment effects.

Results: After 2 weeks the CPAP-withdrawal group experienced a significant increase in the apnoea-hypopnea-index, when compared to the control group (mean change [95\% CI] +31.8 events/hour [ +22.8 to +40.6$], \mathrm{p}<0.001)$. CPAP withdrawal increased levels of DHEA (mean effect $[95 \% \mathrm{CI}]+0.86 \mathrm{ng} / \mathrm{ml}[+0.17$ to +1.35$], \mathrm{p}=0.015)$. Diastolic blood pressure was associated with elevated levels of DHEA (mean effect [95\% CI] +0.05 ng/ml [+0.02 to +0.09$], \mathrm{p}=0.002$ ). The intervention resulted in no statistically significant effects for the other sex hormone levels.

Conclusion: This post-hoc analysis suggests that CPAP withdrawal has an impact on levels of DHEA, while other sex hormones were not affected by the intervention. Changes in DHEA may contribute to the pathogenesis of ED and SD in patients with OSA.

Disclosure: Nothing to disclose. 


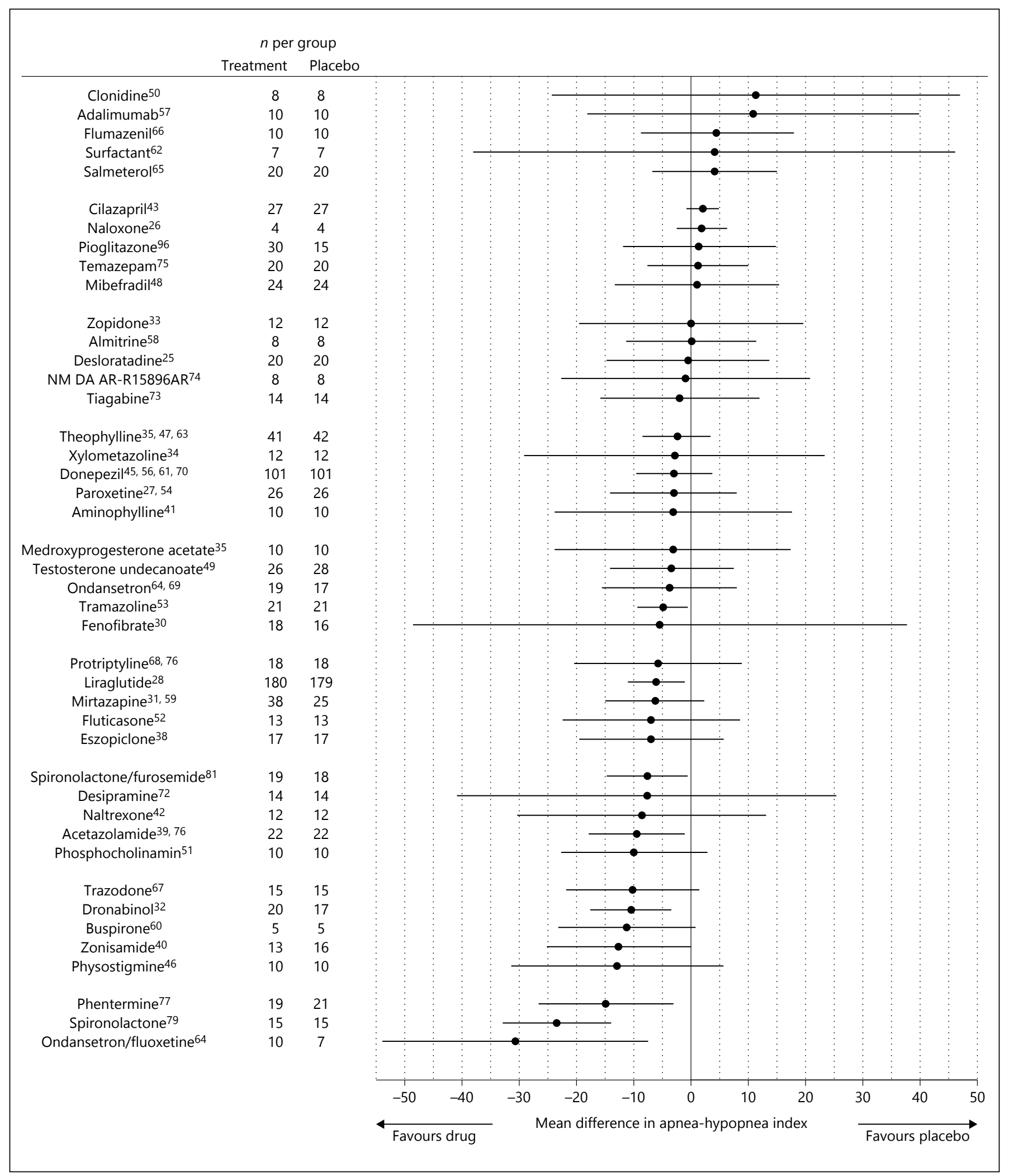

Fig. 1. (for Abstract no P32). 


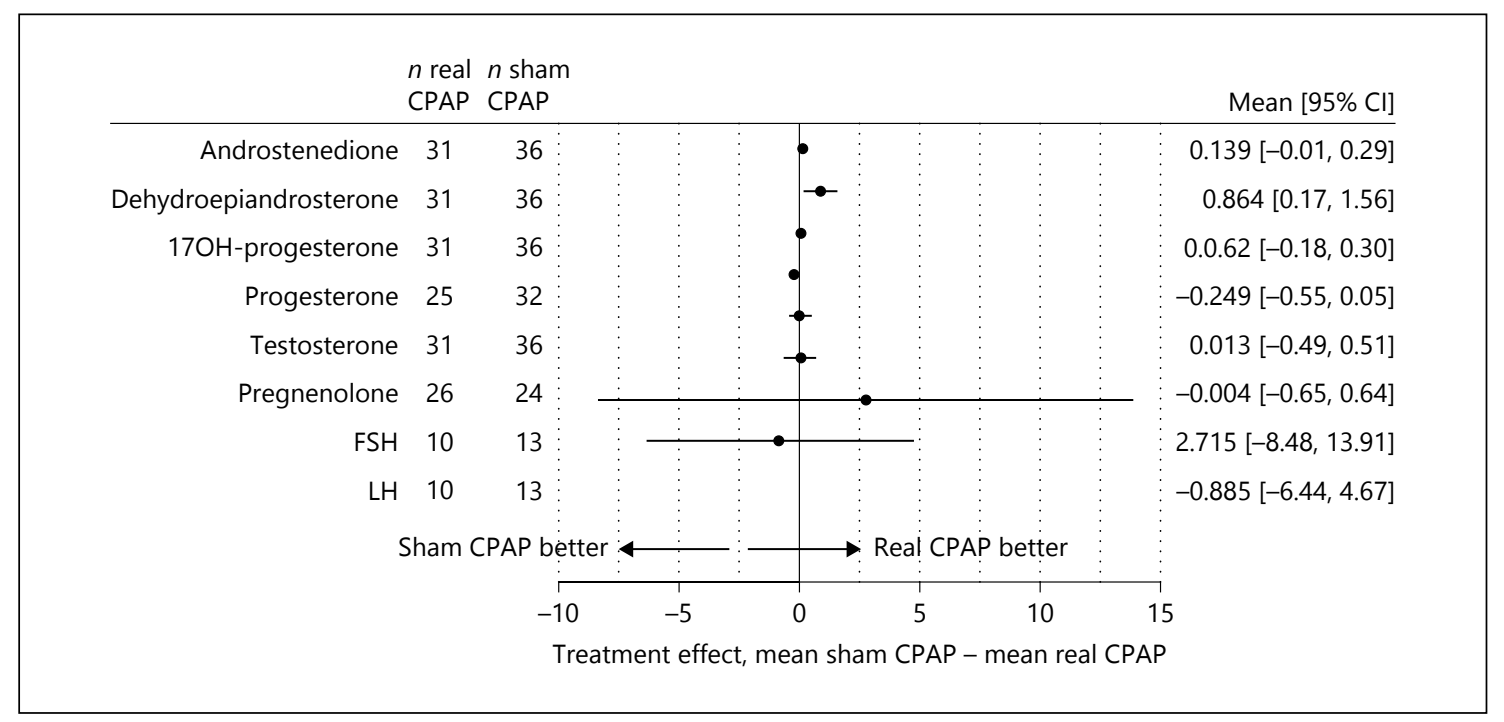

Fig. 1. Effect of CPAP withdrawal (for Abstract no P33).

\section{P34}

\section{The Geneva Lake Study Update: A Descriptive and Cross-Sectional Multicentric Study of Long-Term Home Non-Invasive Ventilation: Deceased Population}

\author{
C. Cantero ${ }^{1}$, P. Pasquina ${ }^{2}$, F. Vienne ${ }^{3}$, A.B. Younossian ${ }^{4}$, M. Prella ${ }^{5}$, \\ P. Gasche ${ }^{2}$, D. Adler ${ }^{2}$, J.-P. Janssens ${ }^{2}$ \\ ${ }^{1}$ Department of General Internal Medicine, ${ }^{2}$ Department \\ of Pulmonology, Geneva University Hospitals, Geneva, \\ ${ }^{3}$ Pulmonary League of Canton of Vaud, Lausanne, ${ }^{4}$ Department \\ of Pulmonology, La Tour Hospital, Geneva, ${ }^{5}$ Department \\ of Pulmonology, Lausanne University Hospital, Lausanne, \\ Switzerland
}

Background: Home noninvasive ventilation (NIV) has increased considerably in our area over the last 20 years. A large database of home NIV was developed in the Cantons of Geneva and Vaud.

Aim: To analyze short-term mortality (rate, risk factors) in patients undergoing long term home NIV.

Methods: Descriptive, multicentric study including all patients undergoing long term ( $>3$ months) home-NIV in the Cantons of Geneva or Vaud. Inclusion started on 01.06.2016 and data base was closed on 10.07.2018. This study analyses all subjects deceased between inclusion and end of observation period.

Results (median, IQR, number and/or percent): 947 patients included in the database, $121(12.8 \%)$ died during study observation period: 53 (44\%) female, mean age $75.6 \pm 8.5$ years, $91(75 \%)$ former and/or active smokers. 41 (34\%) COPD and 16 (13.2\%) Overlap syndrome.

Initiation of NIV: 60 (50\%) emergency setting; 100 (82.6\%) hospital ward. 110 (91\%) had seen pulmonologist within the year prior to inclusion. 108 (89\%) had regular home visits by specialized caregivers.
Median duration of home NIV: 35 months (16-67). Data from ventilator software: compliance $7.2 / \mathrm{h}$, average leaks (95th percentile); $21.6 \mathrm{~L} / \mathrm{min}$, AHI of $1.7 / \mathrm{h}$.

Oxygen added to home NIV: 65 (54\%). Daytime ABG without NIV: $\mathrm{pH} 7.41, \mathrm{PaCO}_{2} 6 \mathrm{kPa}, \mathrm{HCO}_{3} 28.3 \mathrm{mmol} / \mathrm{L}, \mathrm{PaO}_{2}$ room air $(\mathrm{n}=$ $61,50 \%) 8.8 \mathrm{kPa}, \mathrm{SaO}_{2}$ room air $(\mathrm{n}=61,50 \%) 92.5 \%$. Spirometry after bronchodilatation: $\mathrm{FEV}_{1} / \mathrm{FVC}$ : $57 \%, \mathrm{FEV}_{1}: 36 \%$ of predicted.

Conclusion: COPD and Overlap syndrome accounted for the majority of the deceased, with a high rate of former and/or active smokers and being on oxygen supply. Chronic heart failure was more frequent in non-survivors. Average daily use of ventilator and leaks were significantly higher in non-survivors, although differences were clinically minor. Most treatments were initiated during an acute episode and in an inpatient setting, especially in COPD and Overlap syndrome cases.

Disclosure: Nothing to disclose.

\section{P35 \\ Prediction of Sleep Apnea Severity Using a Portable Electrocardiogram Monitoring Device}

\section{F. Baty' ${ }^{1}$, M. Boesch' ${ }^{1}$, S. Widmer ${ }^{1}$, S. Annaheim ${ }^{2}$, P. Fontana ${ }^{2}$, M. Camenzind ${ }^{2}$, R. Rossi ${ }^{2}$, O. Schoch ${ }^{1}$, M. Brutsche ${ }^{1}$ \\ ${ }^{1}$ Lung Center, Cantonal Hospital St. Gallen, ${ }^{2}$ Swiss Federal Laboratories for Materials Science and Technology, St. Gallen, Switzerland}

Introduction: Sleep apnea syndrome (SAS) is diagnosed and graded by polysomnography (PSG) based on the number of apneahypopnea events per hour of sleep (apnea-hypopnea index, AHI). PSG is expensive and technically complex, therefore, simpler and more cost-effective devices for screening and follow-up are desir- 
Table 1. Demographic and clinical characteristics of the patients (for Abstract no P34)

\begin{tabular}{lcc}
\hline Characteristics & Deceased 121 (13\%) & Patients alive 826 (87\%) \\
\hline Age (years), mean (standard-deviation) & $75.6( \pm 8.5)$ & $65.8( \pm 4.2)$ \\
Male, $\mathrm{n}(\%)$ & $68(56.2)$ & $596(72.2)$ \\
Former and/or active smokers, $\mathrm{n}(\%)$ & $91(75.2)$ & $445(53.9)$ \\
Hypertension, $\mathrm{n}(\%)$ & $97(80.2)$ & $563(68.2)$ \\
Obesity, $\mathrm{n}(\%)$ & $52(43)$ & $416(50.4)$ \\
Chronic heart failure, $\mathrm{n}(\%)$ & $35(29)$ & $141(17.1)$ \\
Cerebrovascular disease, $\mathrm{n}(\%)$ & $15(12.4)$ & $81(9.8)$ \\
Modality of initiation of NIV & & $169(20.5)$ \\
$\quad$ Emergency, $\mathrm{n}(\%)$ & $60(49.6)$ & $630(76.3)$ \\
$\quad$ Electively, $\mathrm{n}(\%)$ & $57(47.1)$ & $504(61)$ \\
Setting of initiation of NIV & $100(82.6)$ & $291(35.2)$ \\
$\quad$ Inpatient, $\mathrm{n}(\%)$ & $16(13.2)$ & $304(36.8)$ \\
$\quad$ Outpatient, $\mathrm{n}$ (\%) & & \\
Medical follow-up done by & $46(38)$ & $437(59.9)$ \\
$\quad$ Pulmonologist in hospital center, $\mathrm{n}(\%)$ & $52(43)$ & $85(10.3)$ \\
$\quad$ Pulmonologist in private practice, $\mathrm{n}(\%)$ & $23(19)$ & $629(76.1)$
\end{tabular}

COPD, chronic obstructive pulmonary disease; NIV, noninvasive ventilation; NMD, neuromuscular disease; OHS, obesity-hypoventilation syndrome; Overlap syndrome COPD \& obstructive sleep apnea; SRBD, sleep-related breathing disorder; RD, restrictive disease.

Table 2. Technical aspects regarding ventilators, items provided by ventilator software and monitor (for Abstract no P34)

\begin{tabular}{|c|c|c|}
\hline Duration of use (months), median (IQR) & $35(16-67)$ & $40(18-66)$ \\
\hline \multicolumn{3}{|l|}{ Data from ventlator software: } \\
\hline Leaks at 95th PCTL (L/min), median (IQR) & $21.6(6.6-41.5)$ & $9.6(2.4-24.6)$ \\
\hline AHI (events/hour), median (IQR) & $1.7(0.4-4.5)$ & $1.6(0.5-4)$ \\
\hline Oxygen added to NIV, $\mathrm{n}(\%)$ & $65(53.7)$ & $155(18.8)$ \\
\hline \multicolumn{3}{|l|}{ Daytime ABG without NIV } \\
\hline $\mathrm{pH}$, median (IQR) & $7.41(7.39-7.43)$ & $7.41(7.39-7.43)$ \\
\hline $\mathrm{PaCO}_{2},(\mathrm{kPa})$,median $(\mathrm{IQR})$ & $6(5.3-6.6)$ & $5.6(5-6.3)$ \\
\hline $\mathrm{HCO}_{3},(\mathrm{mmol} / \mathrm{l})$, median (IQR) & $28.3(25.1-31.2)$ & $25.9(24-28.6)$ \\
\hline In room air, $\mathrm{n}(\%)$ & $61(50.4)$ & $320(38.7)$ \\
\hline $\mathrm{PaO}_{2}(\mathrm{kPa})$, median $(\mathrm{IQR})$ & $8.8(7.5-9.4)$ & $9.3(8.4-10.4)$ \\
\hline Yes, $\mathrm{n}(\%)$ & $80(66.1)$ & $479(58)$ \\
\hline \multicolumn{3}{|l|}{ Oxycapnometry under NIV } \\
\hline \%total time $\mathrm{SaO}_{2}<88 \%$ (\%), median (IQR) & $2.8(0-8.1)$ & $0.3(0-8.2)$ \\
\hline Median $\mathrm{PtcCO}_{2},(\mathrm{kPa})$, median (IQR) & $6.3(5.2-6.8)$ & $6(5.4-6.7)$ \\
\hline ODl >3\% (events/hour), median (IQR) & $8.2(4.5-15.7)$ & $6.8(3-13)$ \\
\hline In room air, $\mathrm{n}(\%)$ & $40(33)$ & $209(25.3)$ \\
\hline Median $\mathrm{SpO}_{2}(\%)$, median (IQR) & $93.5(91.4-94)$ & $93.8(91.1-95.3)$ \\
\hline
\end{tabular}

Data obtained in a stable state and not more than 1.2 months prior to inclusion.

ABG, arterial blood gas; $\mathrm{AHi}$, apnea-hypopnea index; COPD, chronic obstructive pulmonary disease; FEV1, forced expiratory volumein one second; FVC, forced vital capacity; IQR, interquartile range; NIV, noninvasive ventilation; NMD, neuromuscular disease; ODI, oxygen desaturation index; OHS, obesity-hypoventilation syndrome; PCTL, percentile; SRBD, sleep-related breathing disorder; RD, restrictive disease. 
able. In this context, a wearable electrocardiogram (ECG) acquisition system was developed by the Swiss Federal Laboratories for Materials Science and Technology (Empa). The aim of the present study was to evaluate the value of overnight ECG recordings to assess apnea severity using an ECG-belt in a population of patients with a suspicion of SAS.

Methods: The ECG-belt consists of a semi-elastic polyester backbone with directly embroidered Ag/Ti-coated PET yarn forming the electrodes. Patients with a suspicion of SAS were recruited. ECG signal was recorded in every patient using both the belt and PSG. Heart rate variability (HRV) analysis was carried out and a prediction model using support vector machines was applied. The prediction accuracy of apnea severity was assessed for both signal acquisition systems.

Results: Whole night measurements were performed in 241 patients. The median AHI was 21 [IQR: $7-40$ ]/h and $65 \%$ of patients suffered from SAS (AHI $>10 / \mathrm{h}$ ). The ECG-belt had an accuracy, sensitivity and specificity of $72 \%, 70 \%$ and $74 \%$, respectively, to discriminate individuals without SAS and individuals with SAS of different degrees of severity. The prediction accuracy was comparable to PSG and particularly suitable for the discrimination between patients with no/mild SAS vs. moderate/severe SAS.

Conclusion: In individuals suspected for SAS a novel textilebased ECG-belt was able to detect HRV-based disturbances of the vegetative nervous system, which allowed for the assessment of sleep apnea severity. The follow-up of patients with SAS could be complemented by HRV, giving additional insights into the cardiovascular stress level during sleep.

Disclosure: Nothing to disclose.

\section{P36 \\ Changing from Oronasal to Nasalpillow Mask May Lower CPAP Pressure and Improve AHI and Adherance in CPAP-Therapy More Than Exspected}

\section{T. Schmid $^{1,2}$, P. Giroud ${ }^{3}$}

${ }^{1}$ Lungenpraxis Stadtgarten Solothurn, ${ }^{2}$ Lungenliga Solothurn, Solothurn, ${ }^{3}$ Heimtherapie, Lungenliga Schweiz, Bern, Switzerland

Background: Oronasal (fullface) masks are often used in CPAP (Continous positve airway pressure), when mouth leak is suspected. However a Meta-Analysis published 2018 in CHEST by Andrade et al. shows that oronasal mask increases CPAP pressure $1.5 \mathrm{cmH}_{2} \mathrm{O}$ and AHI (Apnea Hypopnea Index) 2.8/h and lowers adherance $-48 \mathrm{~min} /$ night in comparison to nasal mask.

Methods/Results: In four patients (\#1-4) with flapping nostrils (inspiratory nasal wing collaps) we saw an even better improvement after changing from oronasal to nasalpillow mask. AHI improved in \#1 from $5.2 / \mathrm{h}$ to $2.1 / \mathrm{h}$, in $\# 2$ from $8 / \mathrm{h}$ to $2.2 / \mathrm{h}$, in $\# 3$ from $11.8 / \mathrm{h}$ to $4 / \mathrm{h}$ and $\# 4$ from $6 / \mathrm{h}$ to $1.6 / \mathrm{h}$ nevertheless the mean pressure was reduced in $\# 1$ from $13.3 \mathrm{cmH}_{2} \mathrm{O}$ to $9.5 \mathrm{cmH}_{2} \mathrm{O}$, in \#2 from $10.8 \mathrm{cmH}_{2} \mathrm{O}$ to $7.3 \mathrm{cmH}_{2} \mathrm{O}$, in $\# 310.3 \mathrm{cmH}_{2} \mathrm{O}$ to $7.3 \mathrm{cmH}_{2} \mathrm{O}$ and in \#4 from $11 \mathrm{cmH}_{2} \mathrm{O}$ to $7.4 \mathrm{cmH}_{2} \mathrm{O}$ in automatic CPAP mode. The CPAP use was improved in \#1 $50 \mathrm{~min} /$ night, in \#2 $123 \mathrm{~min} /$ night in \#3 $93 \mathrm{~min} /$ night and in \#4 $24 \mathrm{~min} /$ night.
Conclusion: Nasal pillow mask improves in comparison with oronasal (fullface) mask the AHI and the CPAP adherance and lowers the mean pressure in automatic CPAP, probably especially in patients with flapping nostrils.

Further studies about the inluence of the different nasal masks (conventional and pillow) in CPAP therapy of patient with flapping nostrils are needed.

Oronasal (fullface) masks should only be used, when leckage can not be compensated by the CPAP machine or when sever patient symptoms due to the leckage are present.

Disclosure: Nothing to disclose.

P37
Anatomical Segmentectomy for Lung Volume
Reduction Surgery After Endoscopic Coils Insertion
S. Federici ${ }^{1}$, J.Martin $^{1}$, A. Lovis ${ }^{2}$, M. Gonzalez'
${ }^{1}$ Division of Thoracic Surgery, ${ }^{2}$ Department of Pneumology,
Centre Hospitalier Universitaire Vaudois (CHUV), Lausanne,
Switzerland

Background: Lung volume reduction (LVR) is a palliative treatment which may improve quality of life and pulmonary functions in patients with severe emphysema. Endoscopic approach by intrabronchial coils insertion has been proposed as alternative for surgery. However, in case of failure of coils, surgery remains technically possible but anatomical lobectomy is generally necessary due to the impossibility to staple parenchyma through the coils.

Methods: Here we present the case of 74-years-old woman who underwent VATS apico-posterior bisegmentectomy of the right upper lobe after previous endobronchial insertion of coils.

Results: A 74-years-old women, with previous smoking was referred for severe COPD. Six years before, she underwent LVR by bilateral intrabronchial coils insertion. After initial improvement, the patient presented progressively worse dyspnea and decreased quality of life. The preoperative pulmonary function tests showed FEV1 of 0.631 (35\% of predicted value) and DLCO (23\% of predicted value) with an associated hyperinflation (residual volume of $251 \%$ of predicted value). Chest CT-scan revealed an heterogeneous emphysema with predominance to the upper lobes. Perfusion scan showed a symmetric perfusion but residual perfusion of $15 \%$ in the anterior segment of the right upper lobe. Previous coils were inserted in the apical and posterior segment of the right upper lobe. The patient underwent a VATS apico-posterior bisegmentectomy of the right upper lobe. Intersegmental plane could be divided with staplers and coils of the upper lobe were all in the specimen. Post-operative course was uneventful and chest tube was removed on day post-operative 3. After post-operative pulmonary rehabilitation, quality of life of patient was improved and the 6-minutes walk distance upgraded from 125 to 245 meters.

Conclusion: Surgery and sublobar resection can be safely proposed in severe COPD patients even after previous intrabronchial coils insertion. Radiological imaging and pre-operative coils localization may help surgeon to decide surgical options.

Disclosure: Nothing to disclose. 


\section{P38}

\section{Clinical Presentation and Cardiovascular Consequences of OSA-COPD Overlap Syndrome}

D. Adler ${ }^{1}$, S. Bailly' ${ }^{2}$ M. Joyeux-Faure ${ }^{2}$, P.M. Soccal ${ }^{1}$, J.-P. Janssens ${ }^{1}$, M. Sapène ${ }^{3}$, Y. Grillet ${ }^{4}$, B. Stach ${ }^{5}$, R. Tamisier ${ }^{2}$, J.-L. Pépin ${ }^{2}$

${ }^{1}$ Service de Pneumologie, Geneva University, Genève, Switzerland, ${ }^{2}$ EFCR Laboratory \& HP2 Laboratory INSERM U1042, Grenoble University, Grenoble, ${ }^{3}$ Nouvelle Clinique Bel-Air, Private Practice, Bordeaux, ${ }^{4}$ Sleep and Respiratory Diseases Center, Private Practice, Valence, ${ }^{5}$ Sleep and Respiratory Diseases Center, Private Practice, Valenciennes, France

How COPD alters clinical presentation of obstructive sleep apnea (OSA) and how the association of both diseases (the overlap syndrome, OVS) impact cardiovascular health remains to be detailed. The primary objective of this study was to report clinical differences at initial presentation between patients with moderate- to-severe OSA and those with OVS. Our secondary objective was to characterize the effect of comorbid COPD on cardiovascular health in OSA population.

We used prospective data from the French Observatoire Sommeil de la Fédération de Pneumologie (OSFP) obtained from January 1997 to January 2017. Among 100,759 registered patients, 72,376 had completed a sleep study at first medical visit and 46,786 had moderate-to-severe OSA. Valid spirometry was available for 16,466 patients.

$14,368(87 \%)$ patients had moderate-to-severe OSA and 2,098 (13\%) patients had OVS. Clinical characteristics of the study population are presented in Table 1.

A lower proportion of OVS patients complained of snoring, morning headaches and excessive daytime sleepiness compared to OSA alone (median ESS score: 9 IQR [6-13] and ESS versus 10 IQR [6-13], $\mathrm{p}<0.02)$. Similarly, a lower proportion of OVS patients $(35.6 \%$ versus $39.4 \%, \mathrm{p}<0.01)$ experienced sleepiness while driving. In contrast, $63.5 \%$ of the OVS population experienced nocturia compared to $58.0 \%$ of the OSA population, $\mathrm{p}<0.01$.

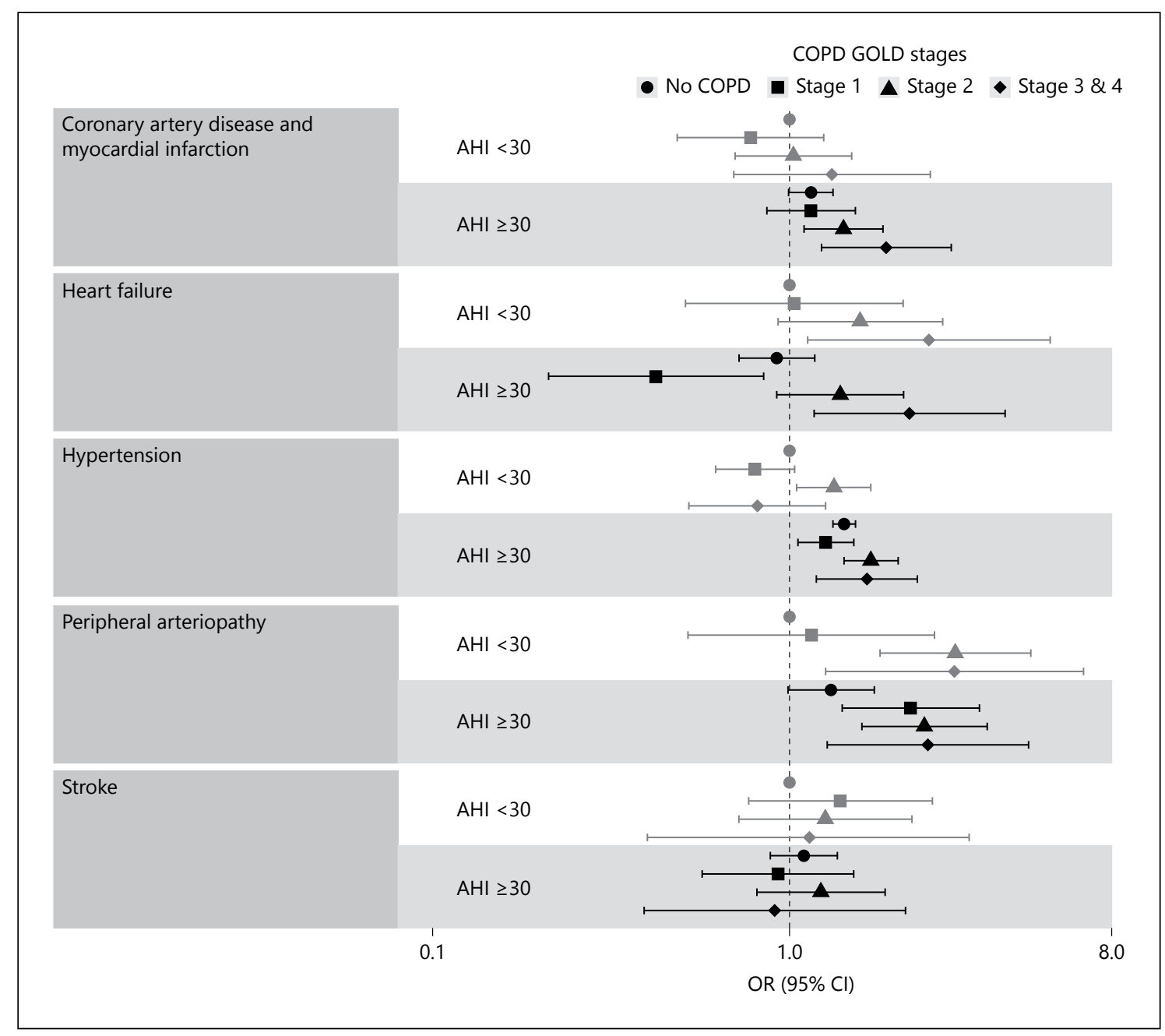

Fig. 1. (for Abstract no P38). 
Table 1. (for Abstract no P38)

\begin{tabular}{|c|c|c|c|c|c|}
\hline Variable & Items & $\begin{array}{l}\mathrm{AU} \\
n=16,466\end{array}$ & $\begin{array}{l}\text { SAS } \\
\mathrm{n}=14,368\end{array}$ & $\begin{array}{l}\text { Overlap } \\
n=2,098\end{array}$ & $\mathrm{p}$ \\
\hline Age (years) & & $57.7[48.6 ; 66.1]$ & $56.9[47.7 ; 65.1]$ & $63.5[55.7 ; 71.7]$ & $<0.01$ \\
\hline Gender (men) & & $11,693(71.2)$ & $10,004(69.8)$ & $1,689(80.7)$ & $<0.01$ \\
\hline Body Mass Index $\left(\mathrm{kg} / \mathrm{m}^{2}\right)$ & & $31.1[27.3 ; 35.9]$ & $31.1[27.3 ; 35.91]$ & $31.1[27.3 ; 35.9]$ & 0.72 \\
\hline \multirow[t]{3}{*}{ Smoking status } & Non-smoker & $8019(48.8)$ & $7316(51)$ & $703(33.7)$ & $<0.01$ \\
\hline & Ex-smoker & $5434(33.1)$ & $4495(31.4)$ & $939(45)$ & - \\
\hline & smoker & $2965(18.1)$ & $2522(17.6)$ & $443(21.2)$ & - \\
\hline Alcohol & & $816(5)$ & $623(4.4)$ & $193(9.3)$ & $<0.01$ \\
\hline Sedentarity & & $3525(21.5)$ & $3014(21.1)$ & $511(24.5)$ & $<0.01$ \\
\hline $\mathrm{FEV}_{1} / \mathrm{FVC}$ & & $81[75 ; 87]$ & $82[77 ; 87.9]$ & $64[59 ; 67]$ & $<0.01$ \\
\hline Apnea + hypopnea Index (event/hour) & & $34[24 ; 50.6]$ & $33.1[23.3 ; 50]$ & $36[25 ; 52]$ & $<0.01$ \\
\hline Oxygen desaturation Index (event/hour) & & $26[14.1 ; 45.2]$ & $25.2[14 ; 45]$ & $28[15 ; 48]$ & $<0.01$ \\
\hline Mean nocturnal $\mathrm{SaO}_{2}$ & & $93[91 ; 94]$ & $93[91.3 ; 94]$ & $92[90 ; 93.8]$ & $<0.01$ \\
\hline \multirow[t]{2}{*}{ Sleep examination } & Polysomnography & $6200(39.8)$ & $5323(39.1)$ & $877(44.1)$ & $<0.01$ \\
\hline & Polygraphy & $9396(60.2)$ & $8285(60.9)$ & $1,111(55.9)$ & - \\
\hline
\end{tabular}

Figure 1 provides a graphical illustration of how alterations in lung function in COPD impacts cardiovascular health in OSA. COPD had no effect on the prevalence of stroke and hypertension. After controlling for confounders, severity of COPD was associated in a dose-response relationship with a higher prevalence of coronary heart disease, heart failure and peripheral arteriopathy.

In adults with moderate-to-severe OSA, comorbid COPD is associated with less symptoms related to sleep-disordered breathing but higher odds for prevalent coronary heart disease, heart failure and peripheral arteriopathy.

Disclosure: Nothing to disclose.

\section{Poster Walk 2: Infection and Pulmonary Vascular Disease}

\section{P39 \\ Clinical Impact of a Multiplex Bacterial PCR of Bronchoalveolar Lavage in Patients with Suspected Lower Respiratory Tract Infection}

\section{A. Salina, D.M. Schumann, K. Jahn, K. Purkabiri, R. Müller, W. Strobel, M. Tamm, D. Stolz}

Clinic of Pneumology and Pulmonary Cell Research, University Hospital Basel, Basel, Switzerland

Introduction: Lower respiratory tract infections (LRTI) are a major cause of morbidity and mortality in patients worldwide. Early pathogen identification is crucial to guide antibacterial therapy and decrease length of hospital stay.
Methods: Prospective, monocentric, clustered, observational study. Patients with suspected LRTI who underwent bronchoscopy with bronchoalveolar lavage (BAL) over 12 months were included. In addition to conventional microbiology, a multiplex polymerase chain reaction (PCR) bacterial assay (Curetis Unyvero) was performed in the BAL on a 2 week on: 1 week off predetermined schedule. No therapeutic recommendation was provided in conjunction with the PCR results to the treating physician. Primary outcome was usage of antibacterial therapy within 30 days after the bronchoscopy.

Results: 605 cases (330 immunosuppressed) were analyzed. When taking into consideration only the 17 pathogens included in the multiplex PCR assay, there was $100 \%$ concordance for positive results between the multiplex PCR assay and conventional culture in 58/107 cases. For negative results, the concordance was $294 / 311$ cases. Thus, conventional culture detected only $54 \%$ of the bacteria evidenced by PCR in the BAL. However, PCR failed to detect bacteria in $5.5 \%$ of the cases with a positive culture.

After bronchoscopy, $57 \%$ (208/368) of the hospitalized patients received antibacterial therapy for LRTI, with an average of $2.1 \pm$ 1.1 antibacterial agents per case used for a median of $10[6 ; 14]$ days. The cases with multiplex PCR results did not have a statistically significant difference in antibacterial therapy length after bronchoscopy ( $p=0.133$ ), amount of antibiotics used after bronchoscopy $(\mathrm{p}=0.357)$ or hospital stay $(\mathrm{p}=0.696)$ compared to cases with only culture results.

Conclusion: PCR detected 50\% more bacteria in the BAL than conventional culture. Without a specific action plan for the treating physician, PCR results of the BAL did not influence antibiotic usage in patients with suspected LRTI.

Disclosure: Nothing to disclose. 


\section{P40 \\ High Prevalence of Peribronchial Focal Lesions \\ of Airway Invasive Aspergillosis in Prolonged Neutropenic Hematological Cancer Patients}

\author{
A. Casutt ${ }^{1}$, J. Couchepin ${ }^{2}$, P.-Y. Bochud ${ }^{2}$, A.-S. Brunel ${ }^{2}$, A. Lovis' ${ }^{1}$, \\ F. Lamoth ${ }^{2}$, C. Beigelman ${ }^{3}$ \\ ${ }^{1}$ Pneumology, ${ }^{2}$ Infectious Diseases, ${ }^{3}$ Radiology, CHUV, \\ Lausanne, Switzerland
}

Invasive pulmonary aspergillosis (IPA) is a life-threatening infection during chemotherapy-induced neutropenia. CT imaging plays an undeniable role in IPA diagnosis, the halo sign being the most suggestive finding of IPA in neutropenic patients. Conversely, peribronchial consolidations/nodules or other non-specific findings are classically described in airway invasive aspergillosis in non-hematological cancer patients. The aim of the study was to characterize chest CT findings of neutropenic hematological cancer patients with proven/probable IPA in a 10-year retrospective cohort.

Hematological cancer patients admitted to the isolation ward of our institution (2007-2017) for myeloablative chemotherapy were retrospectively enrolled if meeting diagnostic criteria of proven/probable IPA (EORTC-MSG definitions) during the neutropenic phase. An expert radiologist reviewed initial CT-scan, corresponding to the timing of IPA diagnosis and all chest lesions were classified using a clinical report form. Results of serum galactomannan at diagnosis and concomitant infections were recorded. Categorical variables concerning galactomannan-positive (GM-P) and galactomannan-negative (GM-N) IPA were studied with the Fisher-test.

35 proven/probable IPA were identified. Peribronchial focal lesions, whether ill-defined consolidations or nodules were observed in 29 IPA ( $83 \%)$. Halo sign was found in 25 IPA (71\%). Hypodense sign was found in 17 IPA (49\%). No air crescent sign was noted. Cavitated nodules were observed in 4 cases $(11 \%)$.

18 proven/probable IPA presented GM-P and 17 presented GM-N. Peribronchial focal lesions were indentified in $16(89 \%)$ GM-P and in 13 (76\%) GM-N IPA. 73 (47\%) peribronchial focal lesions were observed in GM-P and $48(36 \%)$ in GM-N IPA ( $\mathrm{p}$ $0.04)$. No cavitated nodules were identified in GM-P IPA ( $\mathrm{p} 0.04$ ). Bacteriemia was comparable counting for 5 cases (28\%) in GM-P and $4(24 \%)$ in GM-N IPA.

Peribronchial focal lesions, which are classically described in airway invasive aspergillosis, are a common finding in early-IPA whatever the GM value in prolonged neutropenic hematological cancer patients.

Disclosure: Nothing to disclose.

\section{P41 \\ Whole Genome Sequencing for Rapid, Reliable and Routine Investigation of Mycobacterium tuberculosis Transmission in Local Communities}

\section{F. Zakham ${ }^{1}$, A.L. Esteves Carreira ${ }^{2}$, A. Corbaz ${ }^{3}$, E. Masserey', L.P. Nicod ${ }^{2}$, G. Greub ${ }^{1}$, K. Jaton ${ }^{1}$, J. Mazza-Stalder ${ }^{2}$, O. Opota}

${ }^{1}$ Institute of Microbiology, University of Lausanne and University Hospital of Lausanne, ${ }^{2}$ Department of Pneumology, University of Lausanne and University Hospital of Lausanne, CHUV, ${ }^{3}$ Pulmonary Association of Vaud, ${ }^{4}$ Public Health

Department, Canton of Vaud, Lausanne, Switzerland

Background: Contact investigations following the diagnosis of active tuberculosis (TB) is paramount for the control of the disease. Epidemiological data are very powerful for contact tracing but might be delayed and/or difficult to integrate, especially in the setting of multiple contact tracing investigations. The aim of this study was to address the added-value of whole genome sequencing (WGS) to routine local TB surveillance system.

Methods: From November 2016 to July 2017, based on epidemiological data, the local TB surveillance system identified three clusters that could constitute a unique larger outbreak. In a retrospective study, Epidemiological and clinical informations were integrated with genotyping data of $\mathrm{M}$. tuberculosis strains. Whole genome sequences were obtained using a simple DNA extraction method from positive culture coupled with sequencing using an Illumina MiSeq platform. An in-house developed and validated bioinformatics pipeline for single nucleotide polymorphisms (SNPs) analysis was applied for strains genotyping.

Results: Epidemiological investigations identified three putative TB clusters potentially interrelated including 8 patients with active TB. Seven M. tuberculosis isolates were available and analysed by WGS. Using a 5-SNPs threshold to define recent transmission, WGS-based genotyping supported the occurrence of the three clusters (1,2 and 3 ) as well as a link between clusters 1 and 2 $(\mathrm{SNP} \leq 1)$, constituting a larger outbreak. This outbreak was clearly delineated by infirming a potential link with the third cluster (SNP $>500$ ). Genotyping data did not support the belonging of patient 7 to any studied cluster.

Conclusions: This study illustrates the usefulness of M. tuberculosis strains WGS genotyping for routine TB surveillance systems in local communities to rapidly confirm or disprove epidemiological hypothesis and delineate TB clusters especially in the context of multiple contact tracing investigations.

Disclosure: Nothing to disclose. 


\section{P42 \\ Hide and Seek: Repeated Bronchoscopy in Nonresolving Pneumonia}

\author{
N.Pavlov ${ }^{1}$, D. Lin ${ }^{1}$, A. Pöllinger ${ }^{2}$, T. Geiser ${ }^{1}$, M. Funke-Chambour ${ }^{1}$ \\ ${ }^{1}$ Department of Pulmonary Medicine, ${ }^{2}$ Department of \\ Diagnostic, Interventional and Paediatric Radiology, University \\ Hospital Bern (Inselspital), Bern, Switzerland
}

Introduction: Causes of non-resolving pneumonia are various. We report an unusual case of a 46-year old patient with nonresolving pneumonia after antibiotic therapy.

Case Report: A 46-year old female nonsmoker, with history of chronic bronchitis, obesity, arterial hypertension and obstructive sleep apnea, was hospitalized due to right sided chest pain and progressive productive cough after antibiotic treatment for pneumonia for 7 days. Chest CT scan revealed multiple pulmonary infiltrates in the right lower lobe with cavitation and air bronchogram. Flexible bronchoscopy showed hyperplastic mucosa with partial necrosis in the lower right lobe bronchus and subsequent stenosis of the basal segmental bronchi. Mucosal biopsies revealed chronic inflammation with signs of granulation and focal squamous cell metaplasia. Microbial pathogens could not be identified either in the biopsy samples or in the bronchoalveolar lavage fluid. Therefore, an organizing pneumonia was suspected. The patient chose to leave for vacation prior to completion of the diagnostic tests and steroid treatment was not introduced.

One month later, she presented with similar complaints, fever and elevated inflammatory markers. Chest CT scan revealed a persistent pulmonary infiltrate with suspicion of pulmonary empyema, which was confirmed by thoracentesis. Finally, an undetermined plastic foreign body was found in the right lower lobe and successfully removed during repeated flexible bronchoscopy. The patient required thoracoscopic pleural decortication and antibiotic treatment with good clinical recovery. Despite careful history, the origin of the foreign body could not be identified.

Conclusion: There is a variety of causes for nonresolving pneumonia. Foreign body aspiration in adults is uncommon $(0.15 \%-$ $0.25 \%$ ) and up to $50 \%$ of those patients may remain asymptomatic until the development of long-term sequelae. Our case emphasizes the importance of thorough flexible bronchoscopy, if necessary repeated, as a diagnostic tool and its potentially crucial role in the management of a nonresolving pneumonia.

Disclosure: Nothing to disclose.

\section{P43 \\ DLCO as a Marker of Severity in Pulmonary Hypertension}

E. Diamanti ${ }^{1}$, P. Yerly ${ }^{2}$, J.-D. Aubert ${ }^{1}$

${ }^{1}$ Service de Pneumologie, ${ }^{2}$ Service de Cardiologie, CHUV, Lausanne, Switzerland

Introduction: The diffusing capacity of carbon monoxide (DLCO) is reduced in pulmonary vascular diseases. It has been shown that reduced DLCO is observed among elderly patients with idiopathic pulmonary hypertension $(\mathrm{PH})$ with worse functional status and a worse survival. However, DLCO is not included in the risk assessment proposed by the 2015 ESC/ERS guidelines. We aim to investigate the relation between the DLCO and the severity of $\mathrm{PH}$ categories 1, 3 and 4 in the patients' population followed at CHUV.

Methods and Results: We retrospectively reviewed the medical records of consecutive patients seen from 01/2004 to 12/2017. 88 treatment-naïve patients with precapillary $\mathrm{PH}$ confirmed by a right heart catheterization were included and DLCO measurements were recorded.

DLCO is linearly associated with the distance in the 6-minuteswalking-test $(6 \mathrm{MWD})(\mathrm{p}=0.006)$. This relation is stronger for diagnostic categories 1 and 4 . The DLCO is inversely related to the NT-proBNP level $(\mathrm{p}=0.039)$ in category 1 . Overall, a strong relation between DLCO and survival is identified $(\mathrm{p}<0.001)$.

Additionally, we analyzed the potential relationship between the DLCO measurement and the risk stratification, as proposed by the ESC/ERS guidelines. The abbreviated version of the risk assessment was used, as validated by the recent study of Hoeper et al. 65 patients among 88 for whom at least 5 parameters (/6) were available were studied. A trend for lower DLCO values associated with higher ESC/ERS risk was observed $(\mathrm{p}=0.087)$. DLCO and survival were also strongly associated $(\mathrm{p}=0.019)$. By stratifying DLCO in three levels and including into Hoeper's risk assessment, prediction of survival is significantly improved.

Conclusions: DLCO is strongly associated with the walking distance in the 6MWD and lower DLCO value with a worse outcome. This parameter should be included in the initial risk assessment of patients with $\mathrm{PH}$.

Disclosure: Nothing to disclose. 


\section{P44 \\ Internal Validation of MRI Flow Quantification and Correlation to Fick and Thermodilution Catheterization-Derived Cardiac Output}

\author{
L. Crowe', A.-L. Hachulla', S. Noble ${ }^{2}$, L. Genecand ${ }^{3}$, P.M. Soccal', \\ M. Beghetti ${ }^{4}$, F. Lador $^{3}$, J.-P. Vallée ${ }^{1}$ \\ ${ }^{1}$ Service de Radiologie, ${ }^{2}$ Service de Cardiologie, ${ }^{3}$ Service de \\ Pneumologie, ${ }^{4}$ Unité de Cardiologie Pédiatrique, Hôpitaux \\ Universitaires de Genève, Programme Hypertension \\ Pulmonaire, Genève, Switzerland
}

Background: Cardiac output (CO) determination is mandatory for the diagnosis work-up of pulmonary hypertension ( $\mathrm{PH})$. It is classically obtained by invasive methods such as indirect Fick (Qfick) and thermodilution (Qthermo) performed during right heart catheterisation (RHC). Non-contrast, non-invasive and reliable methods may be preferred for clinical routine.

Methods: We compared QFlow derived parameters (stroke volumes and cardiac index/pulmonary and aortic flow) measured non-invasively in $30 \mathrm{PH}$ patients (aged $58 \pm 18$ years) for internal validation of MRI and for comparison to RHC. QFlow was measured by MRI in the major vessels: ascending aorta (AAO), pulmonary artery (PA), right (RPA) plus left (LPA) pulmonary arteries, descending aorta (DAO) plus superior vena cava (SVC); in addition to right and left ventricular cardiac index and stroke volumes (RV, LV). QFick was compared to QThermo, and internal validation of the MRI ( $\mathrm{pp}=\mathrm{Qs}$ ) compared all possible pairings.

Results: Inter- and intra-observer reproducibility was excellent for all MRI flow quantification (flow $\mathrm{L} / \mathrm{min}$ ) ranging from 0.826 to 0.983 and 0.866 to 0.999 , respectively. Internal validation showed that MRI measurements between anatomical sites were accurate and precise. There was a strong correlation of QFlow from MRI with QFick and QThermo with slopes not different from 1, and similarly for the internal validation (a few exceptions were within 0.03 of slope $=1)$. Both stroke volume $(\mathrm{ml})$ and cardiac index/ pulmonary or aortic flow $(\mathrm{L} / \mathrm{min})$ performed well for the internal validation and correlation with RHC $(\mathrm{p}<0.0001)$.

Conclusions: QFlow derived from RHC measurements correlated with QFlow from MRI, and QFlow MRI with internal validation was robust. Stroke volume and flow demonstrated equally good correlations. Due to the precise, reproducible MRI values and strong correlation with QFick and QThermo methods, noninvasive MRI flow quantification may be considered as an alternative to RHC.

Disclosure: Nothing to disclose.

\section{P45 \\ Daily Activity in Incident Patients with Pulmonary Hypertension Is Not Correlated to Hemodynamics But Walk Distance}

S. Saxer, M. Lichtblau, C. Berlier, E.D. Hasler, E.I. Schwarz, S. Ulrich

Pneumologie, UniversitätsSpital Zürich, Zürich, Switzerland

Introduction: The cardinal symptom of pulmonary hypertension $(\mathrm{PH})$ is dyspnea on exertion, which may lead to decreased activity in daily living. The aim of this study is to analyze daily physical activity in incident patients with arterial or chronic thromboembolic $\mathrm{PH}$ (PAH/CTEPH) and to investigate its correlation with pulmonary hemodynamics, symptoms, exercise capacity and other outcomes.

Methods: Incident patients with PAH/CTEPH had a one-week activity assessment by the arm-worn accelerometer SenseWear within -3 months/+2 weeks of the diagnostic right heart catheterization (RHC) and baseline assessments including 6-minute walking distance (6MWD). Activity was correlated to RHC data at rest and exercise and other outcomes.

Results: Thirty-nine PH-patients (24 PAH, $15 \mathrm{CTEPH}, 23 \mathrm{fe}-$ males, $65(54 ; 73)$ years, mPAP $38(30 ; 46) \mathrm{mm} \mathrm{Hg}$, cardiac output (CO) $5.2(4.6 ; 6.3)$ l/min, 6MWD $458(300 ; 593) \mathrm{m}$ were included. $64 \%$ had a sedentary lifestyle ( $<5000$ steps/day), 26 were somewhat active (5000-10,000 steps/day) and 10\% very active. In a multivariate stepwise regression analysis including age, gender, $6 \mathrm{MWD}$ and hemodynamics at rest and exercise (heart rate, mean pulmonary artery pressure, stroke volume), the $6 \mathrm{MWD}$ was the only independent predictor of steps/day ( $\mathrm{B}=16.8(95 \% \mathrm{CI} 11.6-22.0), \mathrm{p}=0.000)$.

Conclusion: Daily physical activity as steps/day assessed in patients newly diagnosed with PAH/CTEPH did not well correlate with invasive hemodynamics at rest nor exercise, but very well with the 6MWD. Whether daily activity assessments provides additional information than pure walk distance on risk factor profiles during follow-up in patients with $\mathrm{PAH} / \mathrm{CTEPH}$ remains to be clarified.

Disclosure: Nothing to disclose.

\section{P46 \\ Validity of Invasive and Noninvasive Methods for Determination of Cardiac Output in Pulmonary Hypertension \\ L. Genecand $^{1,2}$, F. Lador ${ }^{1,2}$ \\ 'Service de Pneumologie, Hôpitaux Universitaires de Genève, Programme Hypertension Pulmonaire, ${ }^{2}$ Faculté de Médecine, Genève, Switzerland}

Background: Direct Fick (DF) is the gold-standard for cardiac output (CO) determination and Thermodilution (TD) is the most widely used one. Besides magnetic resonance imaging and echocardiography, new non-invasive methods would be of interest, in particular for pulmonary hypertension $(\mathrm{PH})$ workup. Several 


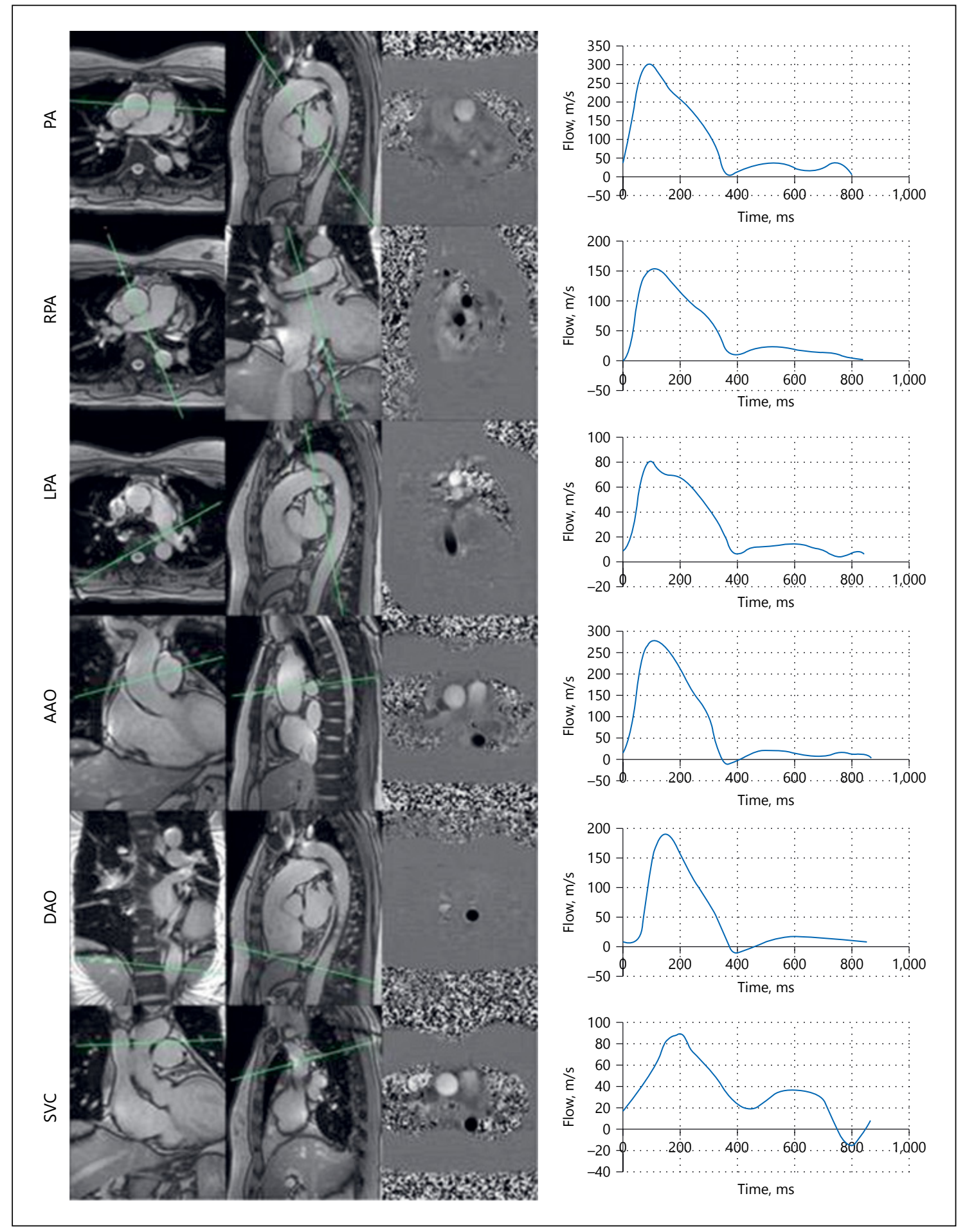

Fig. 1. Positioning of slices on two orthogonal localisers for each of the major vessels (left) and (right) the resulting flow curve (for Abstract no P44). 
Table1. Selected datas of interest concerning invasive CO methods (for Abstract no P46)

\begin{tabular}{llllll}
\hline Studies & 1 & 2 & 3 & 4 & 5 \\
\hline Aim “compare" & COTD and CODF & COTD and CODF & COTD and COIF & COTD and COIF & COTD and COIF \\
Population, $\mathrm{n}$ & 35 & 12 & 198 & 168 & 50 \\
Mean CO, L/min & CODF: 3.7 & CODF: 5.6 & COIF: 5.9 & COIF: 4.2 & COTD: 5.7 \\
& COTD: 3.7 & COTD: 5.3 & COTD: 5.5 & COTD: 4.8 & COIF: 4.8 \\
Bias, L/min & 0.0 & -0.2 & 0.4 & -0.6 & -0.8 \\
LoA, $\mathrm{L} / \mathrm{min}$ & -1.1 to 1.1 & -2.6 to 2.2 & -3.7 and 4.5 & -3.3 to 2.1 & -2.6 to 1.0 \\
PE, $\%$ & \pm 29.7 & \pm 44.0 & \pm 71.9 & \pm 58.7 & 34.2 \\
\hline
\end{tabular}

Table 2. Extraction chart of statistical data used to compare two methods (for Abstract no P46)

\begin{tabular}{|c|c|c|c|c|c|c|c|c|c|c|c|c|c|c|}
\hline & 1 & 2 & 3 & 4 & 5 & 6 & 7 & 8 & 9 & 10 & 11 & 12 & 13 & 14 \\
\hline Bland and Atlman analysis & $\mathrm{Y}$ & $\mathrm{Y}$ & $\mathrm{Y}$ & $\mathrm{Y}$ & $\mathrm{Y}$ & $\mathrm{Y}$ & $\mathrm{Y}$ & $\mathrm{Y}$ & $\mathrm{Y}$ & $\mathrm{Y}$ & $\mathrm{Y}$ & $\mathrm{Y}$ & $\mathrm{Y}$ & $\mathrm{Y}$ \\
\hline $\begin{array}{l}\text { Evaluation of relationship (pattern) between } \\
\text { CO differences and mean CO (proportional } \\
\text { bias) }\end{array}$ & $\mathbf{N}$ & $\mathbf{N}$ & $\mathrm{Y}$ & $\mathrm{Y}$ & $\mathbf{N}$ & $\mathrm{Y}$ & $\mathrm{Y}$ & $\mathbf{N}$ & $\mathrm{Y}$ & $\mathbf{N}$ & $\mathbf{N}$ & $\mathbf{N}$ & $\mathbf{N}$ & $\mathbf{N}$ \\
\hline Assessment of repeatability & $\mathbf{N}$ & $\mathbf{N}$ & $\mathbf{N}$ & $\mathrm{Y}$ & $\mathbf{N}$ & $\mathrm{Y}$ & $\mathbf{N}$ & $\mathbf{N}$ & $\mathrm{Y}$ & $\mathbf{N}$ & $\mathrm{Y}$ & $\mathbf{N}$ & $\mathbf{N}$ & $\mathrm{Y}$ \\
\hline $\begin{array}{l}\text { Assessment of consequence of multiple } \\
\text { measurements }\end{array}$ & $\mathbf{N}$ & $\mathbf{N}$ & $\mathrm{Y}$ & $\mathbf{N}$ & $\mathbf{N}$ & $\mathbf{N}$ & $\mathbf{N}$ & $\mathbf{N}$ & $\mathbf{N}$ & $\mathbf{N}$ & $\mathbf{N}$ & $\mathbf{N}$ & $\mathbf{N}$ & $\mathbf{N}$ \\
\hline $\begin{array}{l}\text { Use of terms: precision, and or accuracy/ } \\
\text { defintions }\end{array}$ & $\mathrm{Y}$ & $\mathrm{Y}$ & $\mathbf{N}$ & $\mathrm{Y}$ & $\mathrm{Y}$ & $\mathrm{Y}$ & $\mathrm{Y}$ & $\mathrm{Y}$ & $\mathrm{Y}$ & $\mathrm{Y}$ & $\mathrm{Y}$ & $\mathrm{Y}$ & $\mathrm{Y}$ & $\mathrm{Y}$ \\
\hline PE calculated & $\mathrm{Y}$ & $\mathbf{N}$ & $\mathbf{N}$ & $\mathbf{N}$ & $\mathbf{N}$ & $\mathrm{Y}$ & $\mathrm{Y}$ & $\mathbf{N}$ & $\mathbf{N}$ & $\mathbf{N}$ & $\mathbf{N}$ & $\mathbf{N}$ & $\mathbf{N}$ & $\mathbf{N}$ \\
\hline I criteria of Interchangeability reached & $\mathbf{N}$ & $\mathbf{N}$ & $\mathbf{N}$ & $\mathbf{N}$ & $\mathbf{N}$ & $\mathbf{N}$ & $\mathrm{Y}$ & $\mathbf{N}$ & $\mathbf{N}$ & $\mathbf{N}$ & $\mathbf{N}$ & $\mathbf{N}$ & $\mathbf{N}$ & $\mathbf{N}$ \\
\hline
\end{tabular}

B\&A: Bland and Altman. 1. Hoeper et al (5), 2. Lador et al (6), 3. Hsu et al (19), 4. Fares et al (20), 5. Alkhodair et al (21), 6. Lador et al (9), 7. Dupuis et al (10), 8. Panagiotou et al (13), 9. Tonelli et al (12), 10 11. Taniguchi et al (11), 12. Rich et al (14), 13. Schwaibimair et al (15), 14. Farina et al (17), 15. McLure et al (16).

innovative methods were studied and sometimes considered reliable. The methodology of such studies may be questionable.

Methods: We evaluated interchangeability of indirect Fick (IF) and TD against DF in precapillary $\mathrm{PH}(\mathrm{PcPH})$. We then analyzed the validity of recently developed non-invasive $\mathrm{CO}$ determination techniques including pulse wave analysis, impedance cardiography, bioreactance and inert gas rebreathing in $\mathrm{PcPH}$. We reviewed the literature using classical database. We included all articles that were comparing invasive or noninvasive methods for $\mathrm{CO}$ determination in $\mathrm{PcPH}$. We used an extrapolation chart systematically looking for 10 statistical end-points to reveal methodological pitfalls. Interchangeability of two $\mathrm{CO}$ measurement methods was accepted when one of the following criteria was reached 1) 2SD of bias $\leq \pm 1 \mathrm{~L} / \mathrm{min}, 2)$ percentage error $(\mathrm{PE}) \leq \pm 20 \%$ when com- paring the tested method to the gold standard DF, 3) $\mathrm{PE} \leq \pm 30 \%$ when comparing the tested method to the reference method TD.

Results: We found 12 articles: 2 comparing DF and TD, 3 comparing IF and TD (Table 1) and 10 comparing non-invasive methods to a reference method. No article reached one of the suggested criteria to accept interchangeability. We observed a lack of standardization of studies comparing CO measurement methods (Table 2).

Conclusions: While TD is largely used in all PH centers, data are scarce and we urge the need for bigger studies evaluating its interchangeability with DF. We do not advise the use of IF. We found no sufficiently reliable non-invasive $\mathrm{CO}$ measurement method. Studies comparing CO measurement methods should use a robust methodology to draw proper conclusions.

Disclosure: Nothing to disclose. 


\section{P47 \\ Right Heart Catheterization Hemodynamics at Rest and Exercise in Supine Versus Upright Position with Focus on Pulmonary Hypertension}

C. Berlier, P. Bader, S. Saxer, M. Lichtblau, E.D. Hasler, E.I. Schwarz, M. Furian, K. Bloch, S. Ulrich

Pneumologie, UniversitätsSpital Zürich, Zürich, Switzerland

Introduction: Body position may influence pulmonary hemodynamics at rest and exercise during right heart catheterization (exRHC), but data is scarce.

Methods: We retrospectively analyzed data of all adults undergoing exRHC from 2005 until 7/2018 due to suspected pulmonary hypertension $(\mathrm{PH})$, with a first cohort undergoing supine exRHC (until 2016) and a second cohort undergoing upright exRHC (2016-2018). Resting hemodynamics between the supine and upright position were compared within subjects of the second cohort (assessed in both positions) and data reported overall and by the following subgroups: no $\mathrm{PH}$ (noPH), $\mathrm{PH}$ only with exercise (exPH), pre-capillary $\mathrm{PH}$, post-capillary $\mathrm{PH}$. Differences in exercise hemodynamics between the supine and upright position were explored by comparing data from the first and second cohort in patients with pre-capillary $\mathrm{PH}$ at maximal exercise.

Results: 270 resp. 213 patients underwent exRHC in the first resp. second cohort $(63 / 60 \%$ female, age $60 \pm 15 / 61 \pm 15$ years, 52/31 noPH, 68/38 exPH, 126/120 pre-capillary $\mathrm{PH}$ and $24 / 24$ post-capillary $\mathrm{PH})$. Supine and upright resting assessments within the second cohort are shown in the Table 1. Comparison of exercise hemodynamics in precapillary $\mathrm{PH}$ between both cohorts revealed that patients achieved higher work rates ( $51 \pm 26$ vs. $28 \pm$ 18 watts) in the upright vs. supine position along with a lower pulmonary arterial wedge pressure $(14.3 \pm 5.2$ vs. $15.8 \pm 5.4 \mathrm{~mm}$ $\mathrm{Hg}, \mathrm{p}=0.007)$, but higher mean pulmonary artery pressure $(63.1 \pm$ 14.8 vs $57.1 \pm 11.8 \mathrm{~mm} \mathrm{Hg}, \mathrm{p}=0.001)$ despite similar cardiac index and resting hemodynamics.
Conclusion: Body position affects resting pulmonary hemodynamics by slightly but significantly reducing mPAP, PAWP, RAP and CI with a $10 \%$ increase of PVR in the upright position. Exercise hemodynamics in patients with precapillary $\mathrm{PH}$ revealed a similar maximal cardiac index, despite a higher workload and transpulmonary gradient in the upright compared to supine position and similar resting hemodynamics. Therefore, accounting for body position is essential in the interpretation of hemodynamics and exercise performance.

Disclosure: Nothing to disclose.

\section{P48 \\ Pulmonary Tumor Thrombotic Microangiopathy: Unusual Diagnosis from Right Heart Catheterisation}

\author{
R. Vongthilath ${ }^{1}$, M. Tognon ${ }^{1}$, A.-L. Hachulla ${ }^{2}$, N. Suh ${ }^{3}$, E. Puget ${ }^{4}$, \\ J.-C. Pache ${ }^{4}$, F. Lador $^{1}$ \\ 'Service de Pneumologie, ${ }^{2}$ Service de Radiologie, Hôpitaux \\ Universitaires de Genève, Programme Hypertension \\ Pulmonaire, ${ }^{3}$ Service des Soins Intensifs, ${ }^{4}$ Service de Pathologie \\ Clinique, Hôpitaux Universitaires de Genève, Genève, \\ Switzerland
}

Pulmonary tumor thrombotic microangiopathy (PTTM) is a rare and severe clinical entity, and it manifests from thrombotic microangiopathy, acute respiratory failure and pulmonary hypertension (PH). This complication of malignancy is mainly diagnosed in autopsies. We describe a case of diagnosis of PTTM made using pulmonary artery wedge blood cell sampling in a patient without relevant past medical history. A non-smoker 85 yo woman with systemic hypertension treated by Amlodipine, presented to our emergency department, because of a ST-segment elevation and suspicion of myocardial infarction. The coronar-

Table 1. (for Abstract no P47)

\begin{tabular}{lllllll}
\hline & & $\begin{array}{l}\text { Overall } \\
\mathrm{n}=213\end{array}$ & $\begin{array}{l}\text { No PH } \\
\mathrm{n}=31\end{array}$ & $\begin{array}{l}\text { Exercise PH } \\
\mathrm{n}=38\end{array}$ & $\begin{array}{l}\text { Pre-capillary PH } \\
\mathrm{n}=120\end{array}$ & $\begin{array}{l}\text { Post-capillary PH } \\
\mathrm{n}=24\end{array}$ \\
\hline mPAP (mm Hg) & Supine & $30(22 ; 40)$ & $18(15 ; 20)$ & $20(18 ; 22)$ & $36(30 ; 46)$ & $33(30 ; 44)$ \\
& Upright & $28(20 ; 38)^{*}$ & $16(12 ; 20)^{*}$ & $19(18 ; 22)$ & $35(28 ; 48)$ & $28(27 ; 34)^{*}$ \\
PAWP (mm Hg) & Supine & $12(9 ; 14)$ & $10(8 ; 10)$ & $9(8 ; 10)$ & $12(10 ; 14)$ & $18(16 ; 22)$ \\
& Upright & $10(8 ; 13)^{*}$ & $8(5 ; 9)^{*}$ & $8(7 ; 10)$ & $12(8 ; 14)^{*}$ & $15(12 ; 17)^{*}$ \\
$\mathrm{RAP}(\mathrm{mm} \mathrm{Hg})$ & Supine & $8(6 ; 11)$ & $8(6 ; 10)$ & $6(5 ; 8)$ & $9(6 ; 12)$ & $11(9 ; 15)$ \\
$\mathrm{Cl}\left(\mathrm{l} / \mathrm{min} / \mathrm{m}^{2}\right)$ & Upright & $6(5 ; 9)^{*}$ & $6(5 ; 8)^{*}$ & $7(5 ; 10)^{*}$ & $7(5 ; 10)^{*}$ & $9(5 ; 12)^{*}$ \\
& Supine & $3(2.5 ; 3.6)$ & $3.6(2.9 ; 4.2)$ & $2.8(2.5 ; 3.4)$ & $2.8(2.5 ; 3.4)$ & $2.9(2.5 ; 3.6)$ \\
PVR $(\mathrm{WU})$ & Upright & $2.8(2.3 ; 3.3)^{*}$ & $3.0(2.7 ; 3.6)^{*}$ & $2.7(2.2 ; 3.2)^{*}$ & $2.7(2.2 ; 3.2)^{*}$ & $2.8(2.5 ; 3.4)$ \\
& Supine & $2.9(1.8 ; 5)$ & $1,3(1 ; 1.5)$ & $4.4(3.2 ; 7)$ & $4.4(3.2 ; 7)$ & $2.6(1.8 ; 4.3)$ \\
& Upright & $3.3(2 ; 5.2)^{*}$ & $1.4(1.2 ; 1.9)^{*}$ & $4.8(3.5 ; 10.3)^{*}$ & $4.8(3.5 ; 10.3)^{*}$ & $2.6(1.8 ; 3.4)$ \\
\hline
\end{tabular}

Values are expressed as median (quartiles). ${ }^{*} \mathrm{p}<0.05$.

$\mathrm{PH}$, pulmonary hypertension; mPAP, mean pulmonary arterial pressure; PAWP, pulmonary arterial wedge pressure; RAP, right atrial pressure; CI, cardiac index; PVR, pulmonary vascular resistance. 

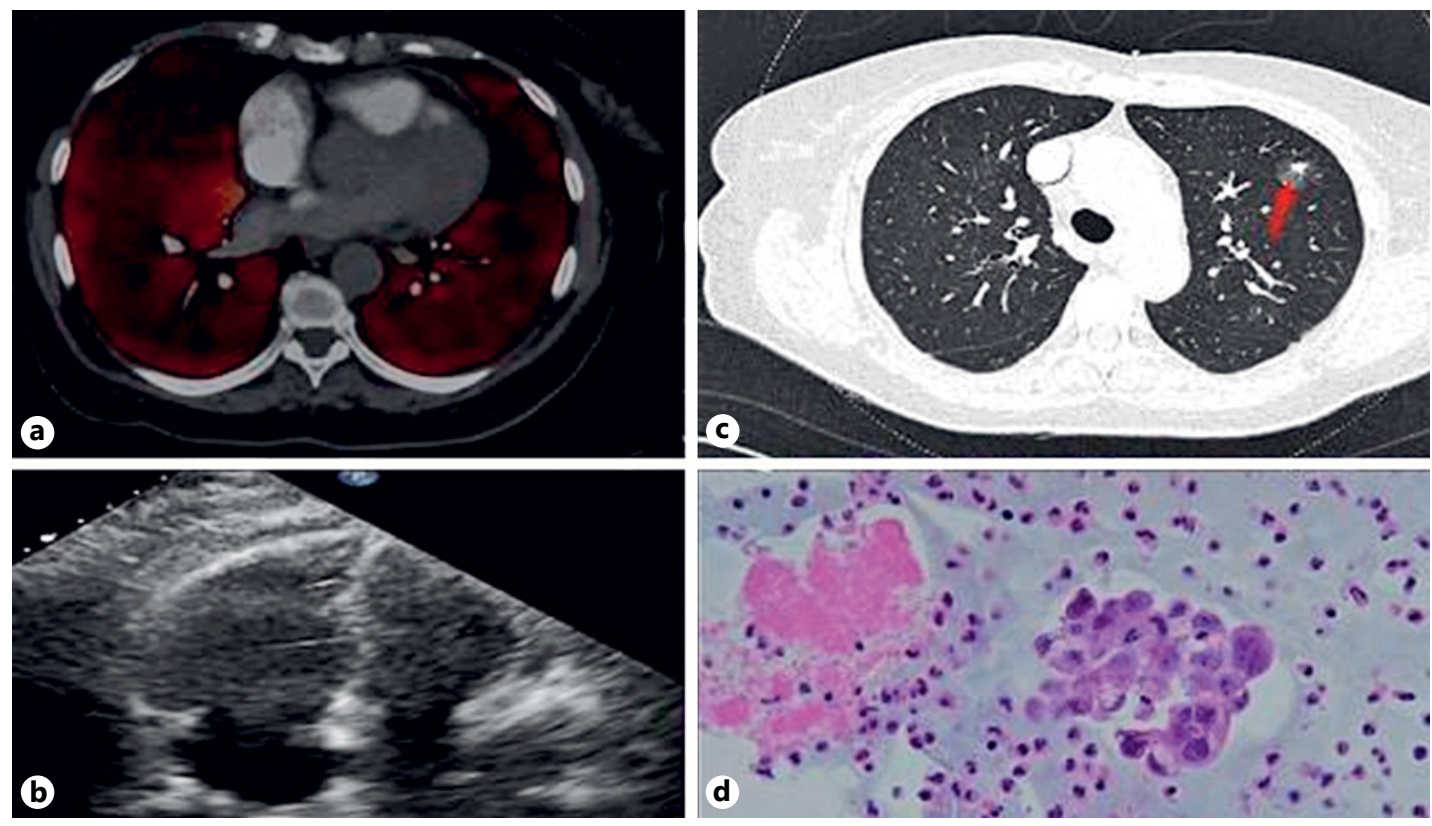

Fig. 1. a No embolism, b TTE with RV dilatation, c nodular opacity (arrow), $\mathbf{d}$ non-small cell carcinoma (for Abstract no P48).

ography excluded any coronary artery disease. Echocardiography demonstrated dilatation and dysfunction of the right ventricle with high probability of $\mathrm{PH}$, no valvular defect and a normal left ventricular systolic and a mild diastolic dysfunction. The chest dual-energy computed tomography showed indirect signs of $\mathrm{PH}$ without thromboembolic lesions and multiple nodular opacities surrounded by a ground-glass attenuation halo. The right heart catheterization showed a severe precapillary $\mathrm{PH}$ with a pulmonary artery mean pressure (mPAP) of $45 \mathrm{~mm} \mathrm{Hg}$, a pulmonary artery wedge pressure (PAWP) of $8 \mathrm{~mm} \mathrm{Hg}$, a $1.5 \mathrm{~L} / \mathrm{min} / \mathrm{m}^{2}$ cardiac index (CI) and severely elevated pulmonary vascular resistance (14 WU). Blood tests for HIV and autoimmunity were negative but revealed a microangiopathic hemolytic anemia, a liver enzymes elevation, severe acute renal failure (clearance $<30 \mathrm{ml} / \mathrm{min} / 1.73 \mathrm{~m}^{2}$ ), an elevated D-dimer (4,167 ng/l) and NT-proBNP (>40,000 ng/l). Finally, cytologic examination of blood aspired from the right heart catheter in wedge position detected circulating non-small cell cancer. Haemodynamic condition rapidly improved and stabilized after management of right ventricular failure with diuretics, dobutamine, noradrenaline, and inhaled nitric oxide. She was subsequently able to get out of ICU one week after admission and introduction of tadalafil. This case highlights the potential diagnostic value of blood cytology using a pulmonary artery wedge catheter as a tool for the diagnosis of PTTM.

Disclosure: Nothing to disclose.

\section{P49 \\ Case Report: Pulmonary Arterial Hypertension Due to Congenital Heart Disease - Think of It in Adults!}

\author{
M. Lichtblau ${ }^{1}$, C. Berlier ${ }^{1}$, M. Greutmann ${ }^{2}$, S. Ulrich ${ }^{1}$
}

${ }^{1}$ Klinik für Pneumologie, ${ }^{2}$ Klinik für Kardiologie, UniversitätsSpital Zürich, Zürich, Switzerland

We present a 42-year old woman with severe pulmonary arterial hypertension (PAH) associated with a congenital heart disease.

A first consultation with a cardiologist took place at the age of 41 after experiencing tachycardia at rest and during exercise for several years. The echocardiography showed significant right ventricular impairment and a systolic right ventricular pressure of about $60 \mathrm{~mm} \mathrm{Hg}$. The diagnosis of precapillary pulmonary arterial hypertension was confirmed in right heart catheterization. The cardiac index and the mixed venous oxygen saturation were high, as well as the shunt fraction in the hyperoxia testing, rising suspicion of a cardiac shunt. A CT-scan as well as a transesophageal echocardiography revealed a superior sinus venosus defect and partial anomalous pulmonary venous connection. A surgical repair (Warden Operation) was performed but mild PAH persisted which was treated with Sildenafil.

Disclosure: Nothing to disclose. 


\section{P50 \\ Comparison of Intrapleural Use of Urokinase and t-PA/DNase in Pleural Infection}

\section{B. Bédat ${ }^{1}$, J. Plojoux ${ }^{2}$, J. Noel' ${ }^{1}$ A. Morel' ${ }^{1}$, J. Worsley ${ }^{1}$, F. Triponez ${ }^{1}$, W. Karenovics $^{1}$}

${ }^{1}$ Thoracic and Endocrine Surgery, ${ }^{2}$ University Hospitals of

Geneva, Geneva, Switzerland

Background: Fibrinolysis can be used to improve fluid drainage in pleural infection. Treatment with either urokinase or tissue plasminogen activator (t-PA) in association with deoxyribonuclease (DNase) via a chest tube has been effective at reducing the need for surgery. This study is the first to compare the efficacy of these two treatments.

Methods: We performed a single-centre controlled prospective cohort study. All individuals with pleural infection admitted to our hospital between January 2014 and December 2017, who were treated with antibiotics, a chest tube, and fibrinolysis, were included in this study. The rate of additional procedure requirements (additional chest tube or surgery) after initial fibrinolysis, complications, costs, and radiological and biological outcomes were analysed.

Results: Among the 93 patients included in this study, 34\% experienced additional procedure after an initial fibrinolysis, including $21 \%$ who received an additional chest tube and $13 \%$ who underwent thoracoscopy. The need for additional procedure arose due to presence of multiple pleural collections $(\mathrm{P}=0.01)$ and was associated with the use of large-bore drain $(\mathrm{P}=0.01)$. The success rate of fibrinolysis was not significantly different between urokinase and $t-P A / D N a s e(P=0.35)$. The difference in drainage duration and in length of hospital stay were not significant as well $(\mathrm{P}=$ 0.05 and $\mathrm{P}=0.12$, respectively). Treatment with $\mathrm{t}-\mathrm{PA} / \mathrm{DNase}$ was cheaper $(\mathrm{P}=0.04)$ but was also associated with a higher rate of haemothorax $(\mathrm{P}=0.002)$.

Conclusion: Treatment with urokinase is safer and equally effective when compared with treatment with t-PA/DNase.

Disclosure: Nothing to disclose.

\section{Poster Walk 3: Interstitial and Vascular Disorders \& Cancer}

\section{P51 \\ Anti-Fibrotic and Apoptotic Effects of Azithromycin in IPF and Control Lung Fibroblasts}

K. Krempaska ${ }^{1}$, S. Barnowski ${ }^{1}$, J. Gavini ${ }^{2}$, N. Hobi ${ }^{3,4}$, A. Stokes ${ }^{1}$, S. Ebener ${ }^{1}$, M. Funke-Chambour ${ }^{1,5}$, T. Geiser ${ }^{1,6}$

'Department for BioMedical Research, Lung Research Laboratory, ${ }^{2}$ Department for BioMedical Research, Visceral and Transplantation Surgery, ${ }^{3}$ ARTORG Center for Biomedical Engineering Research, Organs-on-Chip Technologies, University of Bern, ${ }^{4}$ Alveolix AG, ${ }^{5}$ Department of Pulmonary Medicine, Inselspital, Bern University Hospital, ${ }^{6}$ Department of Pulmonary Medicine, Inselspital, University Hospital Bern, Bern, Switzerland

Rationale: Idiopathic pulmonary fibrosis (IPF) is a lethal lung disease with limited survival. Although new drugs can slow down disease progression, no cure is available. The macrolide Azithromycin (AZT) has anti-inflammatory, immunomodulatory and newly described anti-fibrotic effects possibly via autophagy, but many questions remain unclear. Our study compared anti-fibrotic effects on control and IPF fibroblasts and investigated the mechanisms involved.

Methods: Primary normal human lung and IPF-FB were exposed to TGF- $\beta(5 \mathrm{ng} / \mathrm{ml})$, Azithromycin $(50 \mu \mathrm{M})$ alone or in combination prior to pro-fibrotic gene expression analysis. Control-FB behavior after treatment was screened by microarray analysis. Protein expression was assessed by ELISA and western blot. Apoptosis and intraluminal lysosomal $\mathrm{pH}$ were analyzed by flow cytometry.

Results: AZT treatment significantly reduced collagen 1 secretion in TGF- $\beta$ treated IPF-FB compared to TGF- $\beta$ treatment alone, but not in control-FB ( $\mathrm{p}$-value $\leq 0.01$ ). Addition of AZT compared to TGF- $\beta$ alone did not differ significantly between IPF and controls on gene expression level (Col1, aSMA and CTGF). An increased expression of p62 and LC3II/I protein levels revealed an impairment of the autophagic flux after AZT in both control and IPF-FB. Early apoptosis was significantly higher in IPF-FB compared to controls after AZT treatment (p-value $<0.05)$. Microarray analysis revealed impaired ATP6V1B2 (vacuolar ATPase for lysosome acidification) gene expression. Intraluminal lysosomal $\mathrm{pH}$ was significantly higher after AZT in TGF- $\beta$ treated IPF-FB compared to controls ( $\mathrm{p}$-value $<0.05$ ).

Conclusions: We demonstrate anti-fibrotic properties of AZT in IPF and control-FB, with increased effects on collagen 1 in IPFFB compared to controls. Moreover, AZT induced higher apoptosis in IPF compared to control-FB. Modulation of the lysosomal $\mathrm{pH}$ might contribute to the anti-fibrotic, autophagic and apoptotic effects of AZT in primary fibroblasts from IPF and controls.

Disclosure: Nothing to disclose. 


\section{P52 \\ Standardized Home Inspection in Search of Antigen Exposure in Chronic Hypersensitivity Pneumonitis}

C. Daccord ${ }^{1}$, A. Oppliger ${ }^{2}$, D. Vernez ${ }^{2}$, R. Lazor ${ }^{1}$

${ }^{1}$ Divison of Respiratory Medicine, Lausanne University Hospital - CHUV, Lausanne, ${ }^{2}$ University Institute for Work and

Health - IST, Epalinges, Switzerland

Introduction: Chronic hypersensitivity pneumonitis (CHP) is characterized by an exaggerated immune response to inhaled environmental antigens in susceptible individuals, leading to chronic inflammation and lung fibrosis. Removal of the causal antigen is the cornerstone of therapy and is associated with better outcome. Nevertheless, identification of the source of exposure through medical history is often difficult. To explore the value of standardized home inspection in uncovering the source of antigen, we established a pilot collaboration between the Institute for Work and Health and the Respiratory Medicine Department of the Lausanne University Hospital.

Methods: Patients with a suspected diagnosis of $\mathrm{CHP}$ after routine work-up (history of domestic and occupational exposures using open and standard questions, chest imaging, bronchoalveolar lavage, precipitins, and in some cases lung biopsy) were proposed to have their home inspected in search of antigen exposures. A biologist and occupational hygienist (A.O.) performed a detailed inspection of the home and close environment, using a standard protocol and a questionnaire on patient habits and house/building characteristics. Visible molds were sampled and cultured, and some were tested against patient serum for sensitization.

Results: 19 patients with definite, probable or possible CHP at initial work-up were studied. History of exposure identified possible exposures in $79 \%$, and $63 \%$ had positive precipitins. Home inspection confirmed reported exposures in $32 \%$, and identified new exposures in $37 \%$. Molds were sampled in $58 \%$, and identified by culture in $42 \%$. Altogether, home inspection and/or mold identification provided new information on exposures in $58 \%$, and triggered measures to remove the antigen source in $42 \%$. Poor correlation was found between precipitin positivity and molds identified.

Conclusion: In the setting of suspected CHP, standardized home inspection provides a meaningful contribution to the identification and removal of potentially causative antigens.

Disclosure: Nothing to disclose.

\section{P53}

Thoracic Imaging in Primary Ciliary Dyskinesia

F. Naef ${ }^{1}$, C. Daccord ${ }^{2}$, P. Dumont ${ }^{3}$, M. Meyer ${ }^{4}$, R. Aebi-Di Salvo ${ }^{5}$, P. Eng ${ }^{6}$, N. Howarth ${ }^{7}$, R. Lazor ${ }^{2}$

${ }^{1}$ Faculty of Biology and Medicine, University Lausanne, ${ }^{2}$ Divison of Respiratory Medicine, CHUV, Lausanne, ${ }^{3}$ Department of Medicine, HFR, Fribourg, ${ }^{4}$ Hôpital de la Tour, Meyrin, ${ }^{5}$ Groupe médical, Versoix, ${ }^{6}$ Kinderarztpraxis, Olten, ${ }^{7}$ Department of Radiology, Clinique des Grangettes-Genève, Chêne-Bougeries, Switzerland

Introduction: Few studies have specifically analysed the thoracic imaging features of primary ciliary dyskinesia (PCD) with high-resolution computed tomography (HRCT). The objectives of this retrospective study were to provide a detailed quantitative analysis of chest imaging abnormalities in PCD and to look for correlations with clinical features.

Methods: Cases of PCD were recruited through a Swiss registry for rare lung diseases (SIOLD). Clinical data were collected from medical records through questionnaires. HRCT images were centrally reviewed and scored separately by 2 expert radiologists unaware of clinical data. Discrepancies were resolved by a second consensus reading.

Results: 33 patients with PCD were included (females 64\%, mean age at HRCT $25.9 \pm 15.1$ years). Bronchiectasis were present in $85 \%$. The middle lobe was the most frequently affected (76\%), followed by the lower lobes (64-67\%), whereas the upper lobes (42-48\%) were relatively spared. Lobar atelectasis was found in $24 \%$ and affected only the middle lobe. There was no significant correlation between a bronchiectasis severity score and any clinical characteristics, as well as spirometric variables. Signs of bronchiolitis were common (bronchiolectasis 69\%, tree-in-bud 63\%, centrilobular nodules $72 \%$ ) and co-localized with bronchiectasis.

Conclusion: The middle and lower lobes are the most frequently and severely affected by bronchiectasis in PCD. Signs of bronchiolitis are frequent and co-localize with bronchiectasis.

Disclosure: Nothing to disclose. 


\section{P54 \\ New Comprehensive PCD Diagnostics at the “Interdisciplinary PCD Diagnostic Center PCD-UNIBE"}

\author{
L. Müller ${ }^{1}$, S. Tschanz ${ }^{2}$, E. Kieninger ${ }^{1}$, J.-L. Blouin ${ }^{3}$, A. Jung ${ }^{4}$, \\ P. Latzin ${ }^{1}$, C. Casaulta
}

${ }^{1}$ Pediatric Respiratory Medicine, Department of Pediatrics, Bern University Hospital, University of Bern, ${ }^{2}$ Insitute of Anatomy, University of Bern, Bern, ${ }^{3}$ Laboratory of Genomic and Molecular Diagnostics, Genetic Medicine Division, University Hospitals of Geneva, Geneva, ${ }^{4}$ Pediatric Pneumology, University Children's Hospital Zurich, Zurich, Switzerland

Primary ciliary dyskinesia (PCD) is highly underdiagnosed in Switzerland. With an incidence of 1:10,000, about 800 PCD patients are expected in Switzerland. However, the Swiss PCD registry has only 134 patients identified. One reason for this diagnostic gap could be the challenging PCD diagnosis requiring several standard tests. The current guidelines of the European Respiratory Society (ERS) suggest a multidisciplinary approach involving various methods, including (1) nasal nitric oxide for screening, (2) high-speed-video-microscopy [HSVM] (ciliary beating pattern and frequency), (3) transmission-electron-microscopy [TEM] (ciliary ultrastructure), (4) immunofluorescence microscopy [IF] (ciliary proteins), (5) cultivation of epithelial cells at the air-liquid interface $[\mathrm{ALI}]$, and (6) genetic analysis. Methods 2-5 require living epithelial cells harvested via nasal brushings.

We have newly established a comprehensive, interdisciplinary PCD diagnostic center at the University of Bern (PCD-UNIBE), which offers all diagnostic tools as suggested by the ERS. Genetic analysis is performed by Next-Generation-Sequencing of the entire exome on DNA by the University Hospitals Geneva. During the test phase in 2018, 30 patients were referred to our center; analysis has been completed for 26 cases. We used the fresh samples of all cases for HSVM and cell culture. In $96 \%$ cases, we successfully cultured re-differentiated, ciliated cells. For IF analysis we used fresh and ALI cells in 23 (88\%) and 17 (65\%) cases, respectively. In three cases, there were not enough fresh cells for IF, and we only analyzed ALI cells. We performed TEM analysis in two cases from fresh and five cases from ALI cells. Genetic analysis is now planned in five cases. The cell culture was crucial for a conclusion in 17 (65\%) of the cases and helped to avoid re-brushings of those patients.

Overall, our results show, that despite its complexity and timeconsuming procedures, it is possible to offer this comprehensive diagnostics service in Switzerland.

Disclosure: Nothing to disclose.

\section{P55}

Immunotherapy-Induced Bronchitis/Bronchiolitis: A New Pattern of Lung Toxicity of Immune Checkpoint Inhibitors

\author{
G. Mitropoulou' ', C. Daccord', A. Sauty ${ }^{2}$, B. Egger ${ }^{3}$, A. Pasche 4 , \\ C. Beigelman ${ }^{5}$, I. Letovanec ${ }^{6}$, L.P. Nicod ${ }^{1}$, R. Lazor ${ }^{1}$ \\ ${ }^{1}$ Divison of Respiratory Medicine, Lausanne University Hospital \\ - CHUV, Lausanne, 2Divison of Respiratory Medicine, Pourtalès \\ Hospital - HNE, Neuchâtel, ${ }^{3}$ Divison of Respiratory Medicine, \\ Rolle Hospital - GHOL, Rolle, ${ }^{4}$ Divison of Respiratory Medicine, \\ Morges Hospital - EHC, Morges, ${ }^{5}$ Department of Radiology, \\ ${ }^{6}$ Department of Pathology, Lausanne University Hospital - \\ CHUV, Lausanne, Switzerland
}

Introduction: Immune checkpoint inhibitors (ICIs) have revolutionized the treatment of many cancers, and have proven to be effective in improving overall and progression-free survival. However, ICIs have been associated with a variety of immunerelated adverse events (IRAE), including lung toxicity manifesting as interstitial lung disease and organising pneumonia. We report 2 cases of isolated severe airway disease attributable to ICIs, a previously unreported pattern of lung toxicity.

Case Presentation: Both patients presented with worsening dyspnea and cough while receiving immunotherapy. The first patient participated in a randomized double blind study receiving nivolumab +/- ipilimumab, and the second had been treated with nivolumab for several months. The clinical, imaging and histopathological characteristics are summarized in Table 1 .

An IRAE was suspected based on the temporal relationship between immunotherapy initiation and symptom onset, and exclusion of alternative causes, especially infections. ICIs were stopped in both cases and high-dose prednisone was administered, together with inhaled corticosteroids and bronchodilators. In patient 1, this regimen allowed a rapid clinical and functional improvement, although a repeat course of steroids was required due to transient recurrence of cough and lung function impairment after prednisone tapering. In patient 2 , despite prolonged high-dose prednisone, only a stabilization of FEV1 could be achieved, and disease course was complicated by multiple respiratory infections resulting in further loss of function.

Conclusions: Besides the known pattern of interstitial lung disease, pulmonary IRAE secondary to ICIs may present as bronchitis/bronchiolitis, potentially associated with severe airflow obstruction. ICIs withdrawal and systemic corticosteroids resulted in variable outcomes. Further studies are required to better characterize this new pattern of lung toxicity.

Disclosure: Nothing to disclose. 
Table 1. Patients' characteristics (for Abstract no P55)

\begin{tabular}{|c|c|c|}
\hline & Patient 1 & Patient 2 \\
\hline Sex, age (years) & Female, 65 & Male, 58 \\
\hline $\begin{array}{l}\text { Time from ICIs initiation to first } \\
\text { symptoms }\end{array}$ & 1 week & 13 months \\
\hline Symptoms & Dyspnea, productive cough & Dyspnea, non-productive cough \\
\hline Pulmonary function tests & $\begin{array}{l}\text { Hyperinflation, normal spirometry, } \\
\text { slightly reduced diffusing capacity }\end{array}$ & $\begin{array}{l}\text { Severe airflow obstruction, hyperinflation, air trapping, } \\
\text { severely reduced diffusing capacity }\end{array}$ \\
\hline Histopathology & Eosinophilic bronchitis & Chronic non-specific inflammation, fibrotic remodeling \\
\hline
\end{tabular}

P56

\section{A Single Academic Centre Experience in Lung Cryobiopsies for the Workup of Interstitial Lung Disease}

\author{
A. Kharat ${ }^{1}$, D. Adler ${ }^{1,2}$, B. Bedat ${ }^{2,3}$, X. Montet ${ }^{2,4}$, W. Karenovics ${ }^{3}$, \\ J.-C. Pache ${ }^{2,5}$, K.E. Hostettler ${ }^{6}$, C. Von Garnier ${ }^{7}$, P.M. Soccal ${ }^{1,2}$, \\ J. Plojoux ${ }^{1}$ \\ ${ }^{1}$ Division of Pneumology, Geneva University Hospitals, \\ ${ }^{2}$ Faculty of Medicine, Geneva University, ${ }^{3}$ Division of \\ Thoracic Surgery, Geneva University Hospitals, ${ }^{4}$ Division of \\ Radiology, Geneva University Hospitals, ${ }^{5}$ Department of \\ Clinical Pathology, Geneva University Hospitals, Geneva, \\ ${ }^{6}$ Department of Biomedicine and Clinics of Respiratory \\ Medicine, University Hospital Basel, Basel, ${ }^{7}$ Respiratory \\ Medicine Department, Inselspital, University of Bern, Bern, \\ Switzerland
}

Background: ATS/ERS IPF guidelines give a conditional recommendation to perform surgical lung biopsy (SLB) in patients with non-definitive usual interstitial pneumonia (UIP) pattern on high resolution chest tomography (HRCT). In the absence of specific recommendation for or against lung cryopbiopsies the panel conclude that it is reasonable for experienced centres with a track record to safely continue performing cryobiopsies [1]. We hereby report our experience in lung cryobiopsies in the workup in interstitial lung disease (ILD) patients.

Method: From January 2017 to November 2018, after multidisciplinary discussion (MDD), 11 patients underwent cryobiopsies and 14 patients underwent SLB. Each cryobiopsy procedure was performed with a rigid bronchoscope and prophylactic use of an occlusion balloon. Patient's clinical and functional characteristics are presented in Table 1.

Results: In 10 of the 11 patients undergoing cryobiopsies and in all 14 patients undergoing SLB, our pathologist was able to describe a definitive histopathological pattern. Detailed results regarding length of stay, main complications and diagnostic impact of biopsies are presented in Table 2.

Conclusion: In our experience, cryobiopsies seem to have a promising place in the workup of ILD patients with a positive impact on clinical management. The complications encountered are similar to those described in the literature [2].

Conclusions drawn from our experience are limited by the small number of cases from a single centre which highlights the need for a well-designed national coordinated cohort on ILD in Switzerland.

Disclosure: Nothing to disclose.

\section{References}

1 ATS Documents: Diagnosis of Idiopathic Pulmonary Fibrosis. An Official ATS/ERS/JRS/ALAT Clinical Practice Guideline, September 2018.

2 Herzel J, et al. Transbronchial cyrobiopsies for the Diagnosis of Diffuse Parenchymal Lung Disease: Expert Statement from the cryobiopsy Working Group on Safety and Utility and a Call for Standardization of the Procedure. Respiration 2018;95:188-200. 
Table 1. Clinical and functional characteristics of patients undergoing cryobiopsies or SLB (for Abstract no P56)

\begin{tabular}{lll}
\hline & $\begin{array}{l}\text { Cryobiopsies } \\
(\mathrm{n}=11)\end{array}$ & $\begin{array}{l}\text { Surgical lung biopsies } \\
(\mathrm{n}=14)\end{array}$ \\
\hline Age, median [IQR] & $66[48-78]$ & $72[67-75]$ \\
BMI, kg/m² median [IQR] & $28[26-33]$ & $26[23-28]$ \\
Male sex, n (\%) & $6(55 \%)$ & $10(71 \%)$ \\
FEV1, \% predicted, median [IQR] & $92[81-128]$ & $79[73-85]$ \\
VC, \% predicted, median [IQR] & $88[75-112]$ & $74[65-93]$ \\
TLCO, \% predicted, median [IQR] & $72[59-88]$ & $58[49-70]$ \\
Smoking status, current \& former, $\mathrm{n}(\%)$ & $6(55 \%)$ & $3(21 \%)$ \\
COPD; Coronary artery disease; Diabetes; HBP, $\mathrm{n}$ & $0 / 11 ; 1 / 11 ; 1 / 11 ; 4 / 11$ & $0 / 14 ; 2 / 14 ; 2 / 14 ; 6 / 14$ \\
\hline
\end{tabular}

IQR, interquartile range; BMI, body mass index; FEV1, forced expiratory volume in one second; VC, vital capacity; TLCO, transfer factor of the lung for carbon monoxide; COPD, chronic obstructive pulmonary disease; HBP, high blood pressure.

Table 2. Length of stay, main complications and diagnostic impact of patients undergoing cryobiopsies and SBL (for Abstract no P56)

\begin{tabular}{lll}
\hline & $\begin{array}{l}\text { Cryobiopsies } \\
(\mathrm{n}=11)\end{array}$ & $\begin{array}{l}\text { Surgical lung biopsies } \\
(\mathrm{n}=14)\end{array}$ \\
\hline Length of stay, d median [IQR] & $2[1-2]$ & $3[2-5]$ \\
Fragments biopsied, $\mathrm{n}$ median [IQR] & $4[4-4]$ & $3[2-3]$ \\
Pneumothorax, $\mathrm{n}(\%)$ & $5(45 \%)$ & - \\
Pneumothorax requiring chest tube drainage, $\mathrm{n}(\%)$ & $1(9 \%)$ & - \\
Minor hemoptysis, $\mathrm{n}(\%)$ & $3(27 \%)$ & $0(0 \%)$ \\
Change of initial working diagnosis & $6(55 \%)$ & $8(57 \%)$ \\
\hline
\end{tabular}

\section{P57}

\section{Cisplatin-Resistant Non-Small Cell Lung Cancer Cells Are Characterized by High Levels of Mitochondrial Mass and Are Susceptible to Pemetrexed Treatment}

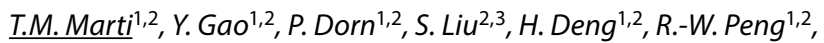 \\ S.R.R. Hall ${ }^{1,2}$, G. Kocher ${ }^{1,2}$, R.A.Schmid ${ }^{1,2}$ \\ ${ }^{1}$ Department of General Thoracic Surgery, Inselspital, Bern \\ University Hospital, Switzerland, ${ }^{2}$ Department of BioMedical \\ Research, University of Bern, Switzerland, ${ }^{3}$ Department of \\ Intensive Care Medicine, Inselspital, Bern University Hospital, \\ Switzerland, Bern, Switzerland
}

Introduction: Lung cancer is the most common cause of cancer-related deaths in developed nations. More than $80 \%$ of lung tumors are non-small-cell lung cancers (NSCLC). Cisplatin plus pemetrexed combination therapy is considered the standard treatment for patients with advanced non-squamous NSCLC. However, advanced NSCLC has a low 5-year survival rate of approximately $30 \%$. Very recently, therapy resistance in hematological and solid tumors has been associated with increased mitochondrial activity.
Thus, the aim of this study was to investigate the role of the mitochondrial activity in NSCLC chemotherapy resistance.

Methods: Mitochondrial mass was determined by MitoTracker staining, which was also used to separate mito-HIGH (10\%) and mito-LOW (10\%) subpopulations from the NSCLC cell line A549 by FACS. Mitochondrial DNA copy numbers were determined by rtPCR. Response to drug treatments was determined by quantification of cell numbers and colony formation assay.

Results: Pemetrexed treatment increased mitochondrial mass in parental A549 cells over time. A549 mito-HIGH subpopulation cells featured a significant increase in mitochondrial DNA copy numbers relative to Mito-LOW cells indicating phenotypically distinct subpopulations can be isolated by our FACS sorting strategy. The proliferation rate of Mito-HIGH cells was significantly increased. Mito-HIGH subpopulation cells was more resistant to cisplatin treatment compared to mito-LOW cells. Interestingly, Mito-HIGH cells were sensitive to pemetrexed treatment compared to Mito-LOW cells.

Conclusion: This study revealed that cisplatin resistant NSCLC cells characterized by high levels of mitochondrial mass and are susceptible to pemetrexed treatment, which could explain the increased efficiency of the combination therapy in the clinical setting.

Disclosure: Nothing to disclose. 


\section{P58 \\ Does Rapid On-Site Cytologic Evaluation During EBUS-Guided Transbronchial Needle Aspiration Reduce the Need for Transbronchial Biopsy in Sarcoidosis?}

$\underline{\text { M. Kolb }}{ }^{1}$, J. Plojoux ${ }^{2}$, P. Gianella ${ }^{2}$, J.-C.Pache ${ }^{3}$, A. Poncet ${ }^{4}$, G. Gex ${ }^{1,5}$, P.M. Soccal ${ }^{1,6}$

${ }^{1}$ Division of Lung Diseases, Geneva University Hospitals, ${ }^{2}$ Geneva University Hospitals, ${ }^{3}$ Division of Clinical Pathology, ${ }^{4} \mathrm{CRC}$ and Division of Clinical Epidemiology, Department of Health and Community Medicine, University of Geneva and University Hospitals of Geneva, Geneva, ${ }^{5}$ Division of Lung Diseases, Hôpital du Valais, Sion, ${ }^{6}$ Faculty of Medicine, Geneva University, Geneva, Switzerland

Background: Endobronchial ultrasound-guided transbronchial needle aspiration (EBUS-TBNA) is an accurate and safe procedure to sample mediastinal tissue for the diagnosis of sarcoidosis among other bronchoscopic procedures as transbronchial biopsy (TBB), endobronchial biopsy (EBB) and bronchoalveolar lavage (BAL). For cancer patients, the use of rapid on-site cytological evaluation (ROSE) during EBUS-TBNA decreases the number of puncture sites and contributes to the collection of sufficient material. When sarcoidosis is suspected, it is not known whether ROSE decreases the need for additional diagnostic interventions. This study compared if, when ROSE was available during EBUS-TBNA, less TBB were performed for the diagnosis of sarcoidosis.

Methods: All data from EBUS-TBNA procedures performed between 2008 and 2014 were prospectively collected. ROSE entered in daily practice in 2011 in our center. In patients addressed with a suspicion of sarcoidosis, we retrospectively analyzed whether ROSE was associated with a reduced use of TBB for suspected sarcoidosis.

Results: Thirty-one patients had a bronchoscopy without and 38 patients had a bronchoscopy with ROSE. Eighty-seven $\%$ and $84 \%$ of patients had a final diagnosis of sarcoidosis. The diagnostic accuracy of EBUS-TBNA was $74 \%$ and $76 \%$ respectively. A mean of 1.6 (SD 0.8 ) and 1.8 (SD 0.8) mediastinal stations were punctured respectively, $\mathrm{p}=0.24$. The need for TBB was of $71 \%$ without ROSE and $50 \%$ with ROSE, which was not statistically significant, $\mathrm{p}=0.129$.

Conclusion: In our cohort, EBUS-TBNA with ROSE did not significantly decrease the need for TBB in patients with sarcoidosis suspicion. Diagnostic accuracy of EBUS-TBNA and number of puncture site was equal in both groups. This negative result should be interpreted cautiously, as the analysis may have been underpowered.

Disclosure: Nothing to disclose.

\section{P59 \\ Electromagnetic Navigation Bronchoscopy for Peripheral Nodules}

K. Jahn ${ }^{1}$, R. Hartmann ${ }^{1}$, D.M. Schumann ${ }^{1}$, J. Bremerich ${ }^{2}$, M. Tamm¹, D. Stolz ${ }^{2}$

${ }^{1}$ Clinic of Pneumology and Pulmonary Cell Research, ${ }^{2}$ Department of Cardiothoracic Radiology, University Hospital Basel, Basel, Switzerland

Introduction: Electromagnetic navigation bronchoscopy $(\mathrm{EMN})$ is a comparatively new technique for the diagnosis of pe-

Table 1. Patients charasteristics (for Abstract no P58)

\begin{tabular}{llll}
\hline & Non-ROSE & ROSE & p value \\
\hline Total patients & 31 & 38 & \\
Female (\%) & $16(52)$ & $18(47)$ & 0.913 \\
Mean age (SD) & $44.2(11.5)$ & $51.5(15.2)$ & 0.026 \\
Final diagnosis (\%) & & & \\
$\quad$ Sarcoidosis/Metastasis/Indefinite/Others & $27(87) / 1(3) / 2(6) / 1(3)$ & $32(84) / 1(3) / 4(11) / 1(3)$ & 0.599 \\
Sarcoidosis stage 1 (\%)/2(\%) & $15(56) / 12(44)$ & $14(45) / 17(55)$ & \\
\hline
\end{tabular}

Table 2. Comparison of diagnostic procedures (for Abstract no P58)

\begin{tabular}{lrrr}
\hline & Non-ROSE & ROSE & p value \\
\hline Number of TBB & $22 / 31(71)$ & $19 / 38(50)$ & 0.129 \\
Number of cytopunction sites & & & \\
$\quad$ Mean (SD) & $1.6(0.8)$ & $1.8(0.8)$ & 0.240 \\
1 station (\%) & $18(58)$ & $16(42)$ & 0.552 \\
2 station (\%) & $9(29)$ & $14(37)$ & \\
3 station (\%) & $4(13)$ & $29 / 38(76)$ & 1 \\
Diagnostic accuracy of EBUS (\%) & $23 / 31(74)$ & & \\
\hline
\end{tabular}


ripheral lung lesions. We analysed the safety and efficacy of EMN in patients admitted to our institution.

Methods: Retrospective study including 175 patients who underwent an EMN at a tertiary instituation.

Results: Data from 175 patients referred to our tertiary institution between 2012 and 2017 were analysed. Average age was 68.0 years with $58 \%$ males and $84 \%$ smokers. Lesion localisation was $57.8 \%$ in the upper lobes, $37.0 \%$ in the lower lobes, and $5.2 \%$ in the middle lobe/Lingula. Nearly $50 \%$ of the lesions were 2 to $4 \mathrm{~cm}$ in size. The average procedure duration was 29.8 minutes. EMN bronchoalveolar lavage, brush, transbronchial needle aspiration, transbronchial biopsy and suction catheter were performed in $92 \%, 89 \%, 74 \%, 75 \%$ and $87 \%$ of the patients, respectively. Minor complications included any desaturation $<90 \%$ (22.9\%), GuedelWendl tube insertion (19\%) and hypertension (8\%). Major complications included heavy bleeding and transfer to intensive care unit $(0.6 \%$, each), premature procedure termination $(1.1 \%)$ and pneumothorax (3.4\%). EMN enabled an affirmative diagnosis in $87(49.1 \%)$ of the cases (18\% (32/175) benign and 31\% (55/175) malignant). Malignancy was diagnosed by CT-puncture or surgery in $52 / 117(44 \%)$ of the patients with negative EMN results. 24 of the 52 patients $(46 \%)$ with false negative EMN results had lesions smaller than $2 \mathrm{~cm}$. These lesions were mainly located in the upper lobes $(63 \%)$.

Conclusion: EMN though relatively safe, was unable to provide an affirmative diagnosis in every second patient.

Disclosure: Nothing to disclose.

\section{P60 \\ Positron Emission Tomography-Computed Tomography (PET-CT) Scan in Evaluation of Microwave Ablation Therapy}

\author{
S. Saenghirunvattana ${ }^{1}$, C. Siangproh ${ }^{1}$, N. Wongkhot $^{2}$ \\ ${ }^{1}$ Pulmonary and Chest Center, Bangkok Hospital Medical Center, \\ Bangkok Dusit Medical Services, ${ }^{2}$ Bangkok Hospital Medical \\ Center, Pulmonary and Chest Center, Bangkok Dusit Medical \\ Services, Bangkok, Thailand
}

Introduction: PET-CT scan is a useful tool to examine treatment response of microwave ablation (MWA) of lung cancer.

Methods: These are reports of three cases of lung cancer: adenocarcinoma stage IV, inflammatory myofibroblastic tumor (IMT) and squamous cell carcinoma stage IV - all treated by MWA then evaluated by PET-CT scan.

Results: First case is adenocarcinoma stage IV on Tyrokinase inhibitor (TKI). After a year of treatment, chest CT scan reported progression of mass. Repeat biopsy confirmed malignancy. Hemoptysis and dyspnea persisted. MWA was performed. Three months post MWA, PET-CT scan revealed the pre-existing hypermetabolic nodule disappeared. The second case is inflammatory myofibroblastic tumor (IMT). MWA was performed on four different occasions on consolidative right upper lung mass. PET-CT scan was done a week after MWA and revealed decreased hypermetabolic pattern of malignant tumor. Three months after MWA,
PET-CT scan revealed metabolic progression of lung mass and tumor regrowth. The last case is squamous cell carcinoma. Chest CT scan reported infiltrative tumor involving entire right lung and right posterior ribs. She underwent tumor debulking, stent placement and five cycles of chemotherapy (Cisplatin and Gemzar) for four months. Follow up Chest CT scan revealed no obvious change in size of right lower lung mass. She agreed to undergo MWA. One month post MWA, PET-CT scan impression was almost total regression of the right lung mass.

Conclusion: The use of PET-CT scan on different timelines post MWA appears to be effective tool in detection of residual disease and assessment of efficacy of procedure.

Disclosure: Nothing to disclose.

\section{P61 \\ mTOR Inhibition in Combination with Chemotherapy Is a Rational Strategy to Treat KRAS-Mutant Lung Cancer}

\author{
R.-W.Peng ${ }^{1}$, S.-Q. Liang ${ }^{1}$, E. Bührer ${ }^{2}$, S. Berezowska ${ }^{3}$, T.M. Marti' ${ }^{1}$, \\ D. Xu ${ }^{1}$, L. Froment ${ }^{1}$, H. Yang ${ }^{1}$, S.R.R. Hall', E. Vassella ${ }^{3}$, Z. Yang ${ }^{1}$, \\ G.J.Kocher ${ }^{1}$, M.A. Amrein ${ }^{2}$, C. Riether ${ }^{2}$, A.F. Ochsenbein ${ }^{2}$, \\ R.A.Schmid ${ }^{1}$ \\ 'Division of General Thoracic Surgery, Department for \\ BioMedical Research (DBMR), Inselspital, Bern University \\ Hospital, University of Bern, ${ }^{2}$ Department of Medical Oncology, \\ Inselspital, Bern University Hospital, ${ }^{3}$ Institute of Pathology, \\ University of Bern, Bern, Switzerland
}

Introduction: Drug resistance inevitably limits clinical efficacy of cancer treatment, which is particularly relevant for KRASmutant cancers, the most common type of human malignancies defined by genetic alterations and the largest subset of tumors that cannot be effectively targeted by currently available therapeutics. Oncogenic KRAS mutations are associated with heightened insensitivity to first-line chemotherapy, but the molecular mechanisms underlying the evolution and persistence of drug-resistant tumors remain poorly understood.

Methods: Various in vitro models that recapitulate KRAS-mutant lung cancer cells evolving acquired resistance to to first-line chemotherapy (cisplatin/pemetrexed) were generated and subjected to pharmacological screens. Tumor vulnerabilities selectively associated with drug-resistant cells were identified and characterized by in vitro, ex vivo and in vivo approaches.

Results: We found that mammalian target of rapamycin (mTOR) pathway is hyperactivated in KRAS-mutant lung cancer cells that evolve resistance to chemotherapy and in patient-derived $K R A S$-mutant lung adenocarcinoma treated with chemotherapy. We further showed that drug-resistant KRAS-mutant lung cancer cells rely on persistent mTOR signaling for survival and that its inhibition restores sensitivity of resistant KRAS-mutant lung cancer cells to chemotherapy. Importantly, drug combinations of clinically approved mTOR inhibitors with chemotherapy synergize in inhibiting cell proliferation of KRAS-mutant cancer cells in vitro and in vivo. These results pinpoint $\mathrm{mTOR}$ as a mechanism 
of resistance to chemotherapy in KRAS-mutant lung cancer and validate a rational and readily translatable strategy to treat $K R A S$ mutant lung cancer.

Conclusions: Our study identifies a novel mechanism of resistance mechansim to chemotherapy and validates a combination strategy to treat KRAS-mutant lung cancer.

Disclosure: Nothing to disclose.

\section{P62 \\ Early Detection and Resection of Lung Cancer by Electromagnetic Navigation Bronchoscopy with Dye-Marking}

\section{S. Saenghirunvattana, C. Suwanakijboriharn, P. Tangpiroontham, C. Siangproh, N. Wongkhot}

Bangkok Hospital Medical Center, Pulmonary and Chest Center, Bangkok Dusit Medical Services, Bangkok, Thailand

Introduction: Dye-marking through the guidance of Electromagnetic Navigation Bronchoscopy (ENB) followed by thoracsocopic surgery is an innovative approach to increase success rate of early diagnosis and treatment of lung cancer.

Method: This case report describes the technique used to localize a lung lesion to aid surgeon in resection of early stage of lung cancer.

Result: This is a case of a 79-year-old female, non-smoker, hypertensive patient with lung lesions. Her chest CT scan reported multiple small ground glass opacity nodules in peripheral zone of right upper lobe (RUL), right lower lobe (RLL), left upper lobe (LUL) and one $9.2 \mathrm{~mm}$ solid nodule at posterior zone of right lower

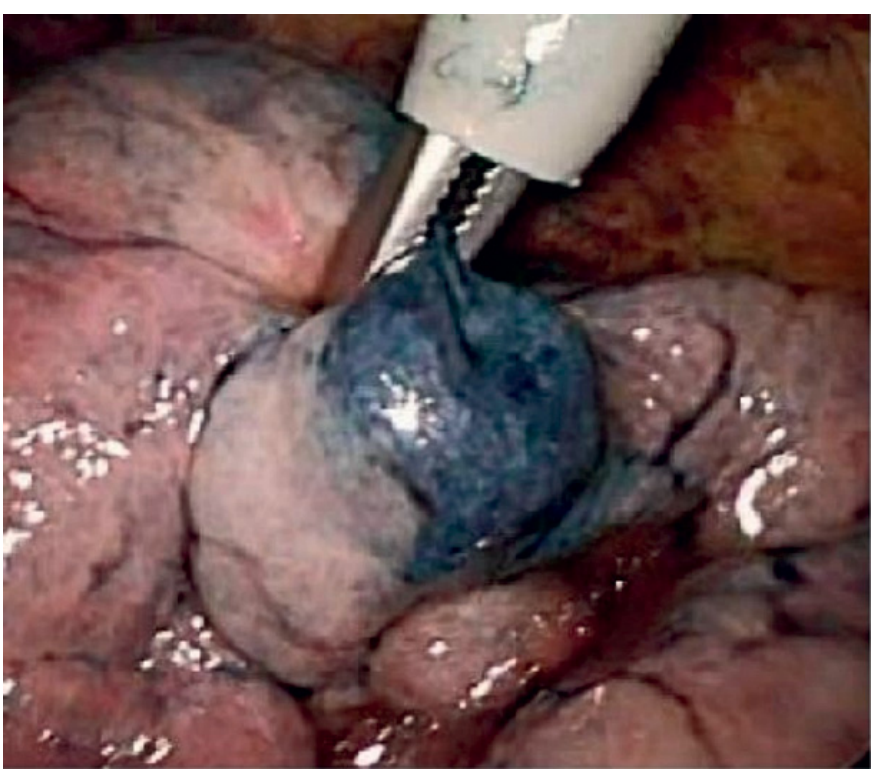

Fig. 1. Lung lesion with dye-marking for excisional biopsy (for Abstract no P62). lobe (RLL). PET-CT scan revealed tiny nodules in both lungs are too small to take up the FDG. ENB with dye-marking was performed by first uploading patient's chest CT scan into the planning software to create a pathway to locate the lung lesions. In the operating room, an electromagnetic field was created through the location board placed under the patient mattress. The patient was then sedated. Airway was cleared by regular bronchoscopy. Following the pre-designed plan, the extended working catheter with locatable guide wire was directed to the target lesion. Once in position, the guide wire was removed and a bronchosocpic needle gauge 25 with pre-loaded $0.3 \mathrm{ml}$ of methylene blue was inserted through the catheter then methylene blue was injected. The procedure was then repeated to the other nodules. Then, the patient was properly positioned and draped to undergo wedge resection of all the lung nodules. Frozen section revealed Adenocarcinoma only at the RLL (stage I). RLLL lobectomy was then performed after.

Conclusion: Early detection and excision of lung cancer can be done by CT scan of the chest, aided by electromagnetic navigation bronchoscopy and dye-marking.

Disclosure: Nothing to disclose.

\section{P63}

\section{Congenital Alveolar Dysplasia}

\section{$\underline{\text { S. Guérin }}{ }^{1}$, A. Mornand ${ }^{2}$, I. Ruchonnet-Metrailler' ${ }^{2}$, C. Barazzone ${ }^{2}$, I. Rochat ${ }^{1}$, S. Blanchon ${ }^{1}$ \\ ${ }^{1}$ Pneumologie Pédiatrique, CHUV, Lausanne, ${ }^{2}$ Pneumologie Pédiatrique, HUG, Genève, Switzerland}

We report the case of a girl born at 40 weeks of gestation, after a normal pregnancy including normal fetal ultrasounds and no mother medication. At birth, amniotic fluid was meconiumstained and the newborn rapidly developed a severe respiratory distress with pulmonary hypertension despite repeated endotracheal suctions, finally requiring veno-arterial extra-corporeal membrane oxygenation (ECMO). Cardiac ultrasound showed an isolated patent ductus arteriosus, ligated on day 5. Lung biopsy was done at the same time. Histological analysis showed $95 \%$ of immature lung tissue as septa were overly thick and capillary network immature, corresponding to the saccular stage of lung development. There was no misalignment of pulmonary veins. Dander and hair were found in interstitial tissue, confirming meconial aspiration. Intravenous steroid treatment led to slow respiratory improvement. ECMO was weaned on day 16, invasive ventilation on day 26 and supplemental oxygen on day 75. At 3.5 months, she was eating and growing perfectly well and showed residual isolated tachypnea (40/min), normal breathing sounds and oxygen arterial saturation.

The severity of the initial respiratory distress was related to massive meconial aspiration on immature lungs. Congenital alveolar dysplasia was suspected, an extremely rare condition considered as genetic without any precise gene involved to date. Literature is poor, prevalence is unknown but probably underestimated as lung biopsy is rarely performed. The prognosis looks severe given the publication of only one surviving case. We can hypothesize that the remarkable recovery of our case is explained by a less severe 
congenital alveolar dysplasia than those described in literature, regarding the stage of development or possibly the extent of the lung involved, which assess was impossible with only one small lung biopsy.

Disclosure: Nothing to disclose.

\section{Poster Walk 4: Prevention, Diagnostic Techniques, Clinical Pearls}

\section{P64 \\ Text Mining - A Tool for Quality Assessment of Medical Records}

\section{J. Hegermann F. Baty, M. Brutsche}

Lung Center, Cantonal Hospital St. Gallen, St. Gallen, Switzerland

Introduction: Electronic medical records constitutes an important source of information for large scale healthcare quality studies. Text mining can be applied to automate knowledge extraction from unstructured data included in medical records and generate quality indicators applicable for medical documentation. The objective of the current study was to apply text mining methodology for the analysis of polysomnographic medical reports in order to assess the precision and the inter-physician variability in the diagnostic evaluation of sleep-disordered breathing.

Methods: Polysomnography reports of 243 sleep investigations scored by 9 trained sleep physicians from the respiratory sleep team of the Sleep Center St. Gallen were analyzed using a text mining approach. Diagnoses included obstructive, central and mixed sleep apnea with various degrees of severity. After filtering for meaningful discriminating terms, a term-document matrix was generated and analyzed using correspondence analysis.

Results: Diagnosis descriptions from the 243 polysomnography reports provided 49 discriminating terms which were used in our analysis (Fig. 1a). Patterns in the usage of these terms allowed for the characterization of patients' type of disease (Fig. 1b) and disease severity (Fig. 1c). The inter-physician homogeneity was assessed and revealed significant variations among physicians (Fig. 1d).

Conclusion: Text mining could be used to optimize the quality, as well as the precision and homogeneity of medical reporting of diagnostic procedures - here exemplified with polysomnography.

Disclosure: Nothing to disclose.

\section{P65 \\ Transition from Pediatric to Adult Center in Cystic Fibrosis: Which Consequences for the Patients?}

\author{
C. Despland', B. Touilloux ${ }^{2}$, J.-C. Suris ${ }^{3}$, L. Nicod ${ }^{2}$, M.Prella $^{2}$ \\ ${ }^{1}$ University Lausanne, ${ }^{2}$ Pneumology Unit, ${ }^{3} \mathrm{IUMSP}, \mathrm{CHUV}$, \\ Lausanne, Switzerland
}

Introduction: In cystic fibrosis (CF) life expectancy has increased considerably over the last two decades. The number of patients followed in adult centers raises regularly. The transition from the pediatric team to the adult center has become important to ensure the quality of care and prognosis.

The purpose of this study is to evaluate the impact of this transition on medical and psycho-social aspects of the disease.

Methods: In this retrospective study we included the last 15 patients who were transferred from the $\mathrm{CF}$ pediatric team to our adult center. We looked in the medical files and compared medical, social and educational variables at the time of transfer (t1) and two years later ( $\mathrm{t} 2)$.

Results: Table 1 shows the medical situation at $\mathrm{t} 1$ and $\mathrm{t} 2$. The analysis of the body mass index (BMI) curves showed that $53 \%$ of patients slowed down their growth from $t 1$ to $t 2$. The presence of a severe liver injury (cirrhosis or liver transplant) and/or diabetes was a risk factor for it. Bronchial obstruction got worse in $40 \%$ of patients. The therapeutic compliance did not vary, but the regularity of the follow-up by the CF center decreased in adult medicine (regular follow-up $73 \%$ at $\mathrm{t} 1,47 \%$ at $\mathrm{t} 2$ ). At $\mathrm{t} 1$, all patients were either in school or in apprenticeship with an $100 \%$ activity rate for $93 \%$ of them. At $\mathrm{t} 2$, we found the same activity rate for the patients still in school or in apprenticeship, but among the 8 others $63 \%$ were unemployed.

Conclusion: The nutritional and respiratory health deteriorates in parallel with a decrease in the regularity of the medical follow-up after the transfer. Integration into a workplace is more difficult than integration into the school environment. The transition process should pay particular attention to these points.

Disclosure: Nothing to disclose.

Table 1. Age and medical characteristics of the 15 patients (for Abstract no P65)

$\begin{array}{ll}\text { At transfer } & 2 \text { years } \\ (\mathrm{t} 1) & \text { later }(\mathrm{t} 2)\end{array}$

Age - years

$17(17-18)-19(19-20)$

FEV1 $<80 \%$ - number of patients

$\begin{array}{cc}17(17-18) & 19(19-20) \\ 3 & 6 \\ 2 & 5 \\ 7 & 7\end{array}$




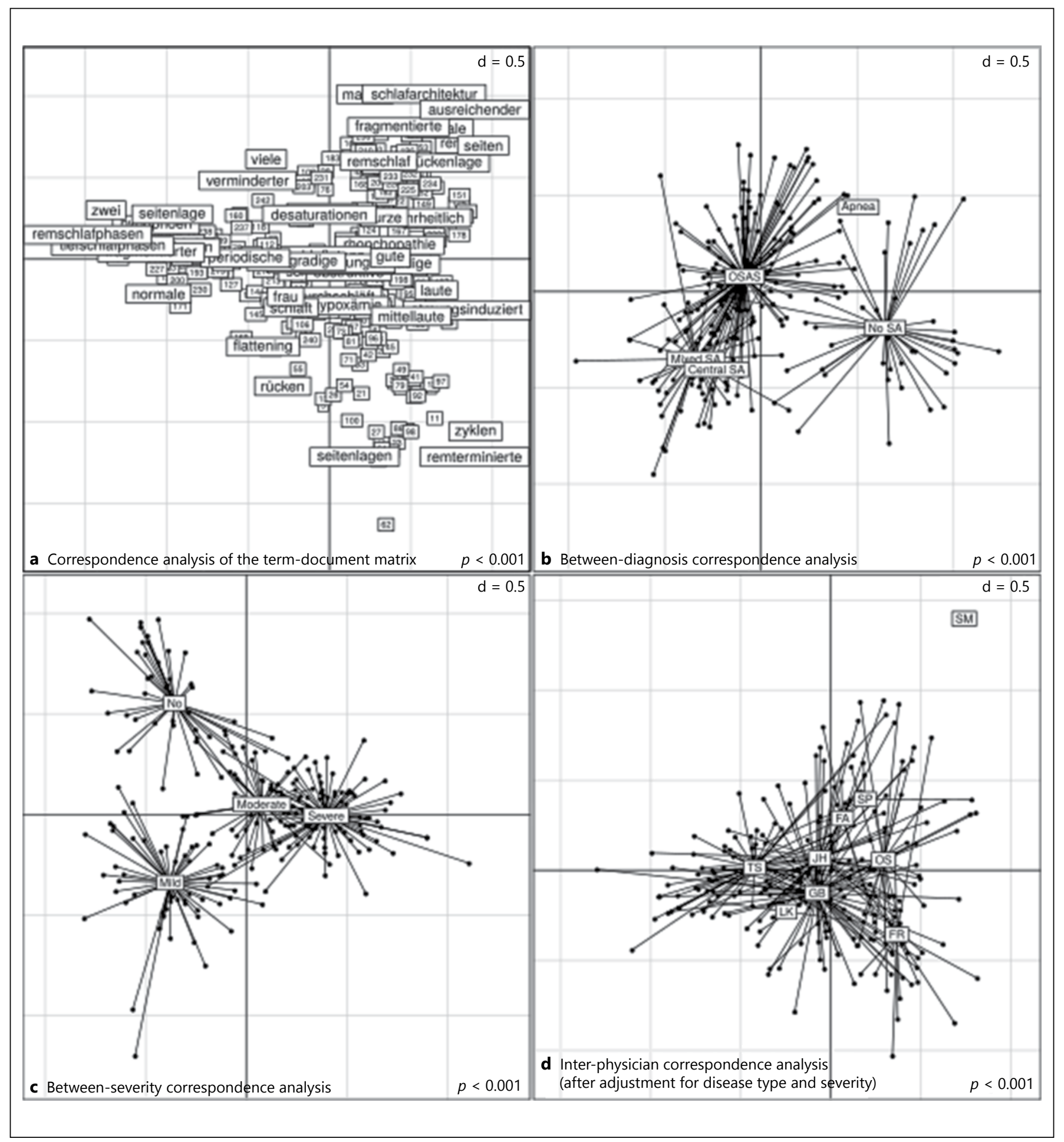

Fig. 1. (for Abstract no P64). 


\section{P66 \\ Information, Content Marketing, Official Communication Around E-Cigarette "Juul"'s Introduction into the Swiss Market}

\section{$\underline{\text { R. Kaelin }}$}

Private Practice, Oxyromandie/OxySuisse, Etoy, Switzerland

Background: Despite causing nicotine (N) addicts in USA, "Juul" was admitted 12/2018 into the $\mathrm{CH}$-market without any legal action by the government. Exceptions for "alternative products" (AP) in the future Tobacco (T) products law (TabPG) and Philip Morris' (PM) claim "to end the cigarette" facilitated this.

Method: Search publications minimizing addiction risk and need for advertising bans including AP.

Results:

- NZZ 15.04.2017 „Alternativen zum blauen Dunst“. AP’s are favored

- Le Temps 30.05.2017 „Le vapotage réduit les risques de 95\% ».J.Fr.Etter interviewed.

- NZZ am Sonntag 19.11.2017.: „Das kleinere Uebel“.

- Press release 22.11.2017: „Verdampfen statt Verbrennen. Suchtfachleute fordern Kurswechsel in der CH-T-politik“. No bans mentioned.

- NZZ 22.11.2017: "Suchtverbände sind für die E-zigarette"

- NZZ 27.11.2017: „Die Schweiz beginnt bei der Tabakprävention umzudenken“. PM-Sponsored content

- NZZ «Die Zukunft ohne Rauch beginnt in der $\mathrm{CH}^{\text {“.27.11.- }}$ 20.12.2017 content marketing nzz.ch/PM.

- Blick.Website 2.07.2018: «430 Forscher tüfteln am Ende der Zigarette». Hidden marketing denounded to Swiss Press council.

- Press release 30.11.2018: „Tabakwerbung Nein. Botschaft zum TabPG: Schweiz darf nicht Schlusslicht bleiben“. Association of prevention pledges ad bans, but not for AP.

- Blick am Abend 05.12.2018. "Wollen nicht die gleichen Fehler wie in den USA machen“. Juul labs claims not targetting the youth and to refrain from sales to $<18 \mathrm{y}$.

- Hospital Tribune 27.12.2018: «L'histoire de la lutte anti-tabac nous suggère qu'il n'y aura peut être jamais consensus» $\mathrm{R}$. West does'nt mention $\mathrm{N}$ addiction risk in youth, nor ad bans for E-cigs

- Keystone SDA/ATS: 28.12.2018: «L'e-cig se fait une place malgré la controverse». The federal office is pleased by autoregulation. Ad bans not mentioned.

Conclusion: Synergy of media reports, content marketing and official communications favored "harm reduction" over the need for ad bans to prevent addiction in youngsters.

Disclosure: Nothing to disclose.

\section{P67 \\ A Case of Microwave Ablation of Lung Tumor in a Patient with Implanted Pacemaker}

\section{S. Saenghirunvattana, P. Wetchanien, C. Siangproh, N. Wongkhot}

Pulmonary and Chest Center, Bangkok Hospital Medical Center, Bangkok Dusit Medical Services, Bangkok, Thailand

Introduction: Microwave ablation (MWA) heats lung tissue by creating an electromagnetic field in the target area around an ablation device. There are varying reports as to whether thermal ablation energy has procedure-related effect on cardiac devices. The purpose of this case study is to report that MWA is a successful non-invasive procedure to patients with pacemaker implants.

Method: A case report of MWA of a progressive lung tumor to a patient with pacemaker implant.

Result: A case of an 83-year-old female patient diagnosed of Adenocarcinoma of sigmoid colon, status post laparoscopic sigmoidectomy. She received chemotherapy (Xeloda) but stopped intermittently due to rising creatinine levels. 2 years after initial diagnosis, chest $\mathrm{CT}$ scan reported two lung nodules at right lower lobe $(1.1 \mathrm{~cm})$ and another at left upper lobe $(0.7 \mathrm{~cm})$. Biopsy revealed Adenocarcinoma. Carcinoembryonic antigen (CEA) levels continuously increased even with chemotherapy. Repeat PET-CT scan after another 2 years revealed progression of lung metastases of right upper lobe from $1.0 \mathrm{~cm}$ to $1.8 \mathrm{~cm}$. She has the following underlying conditions: Hypertension, Congestive Heart Failure, Complete AV block with junctional escape s/p DDDR pacemaker. Microwave ablation therapy was advised as an alternative noninvasive treatment of lung cancer. Anti-coagulants were stopped for a week prior the first session of MWA. Under CT guidance,

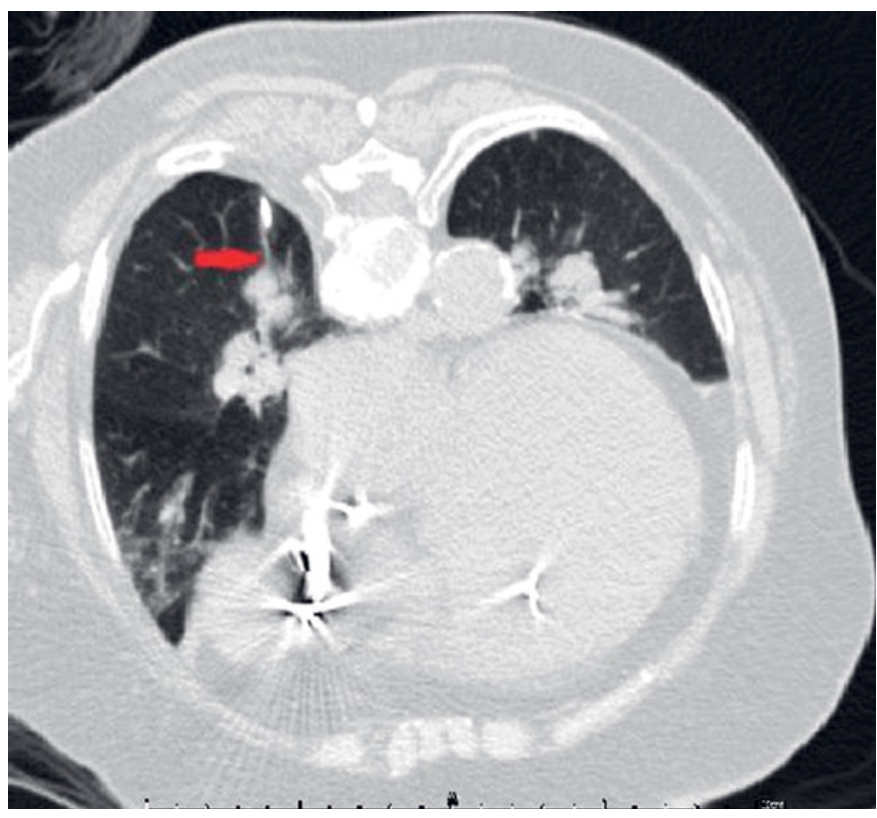

Fig. 1. Chest CT scan image of MWA needle into the left upper lung nodule (for Abstract no P67). 


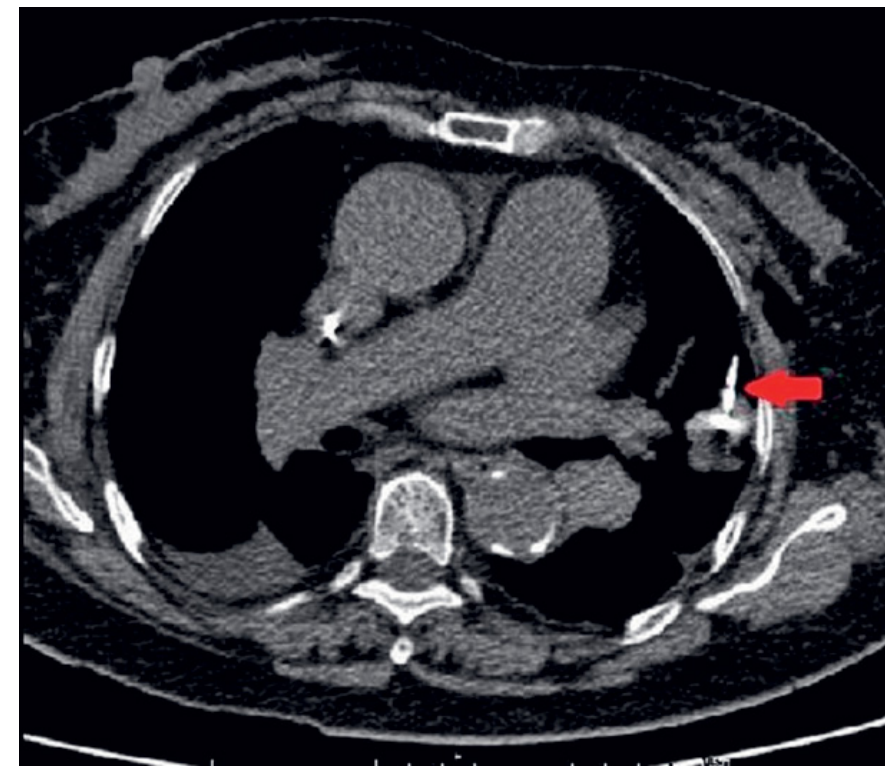

Fig. 2. Chest CT scan image of MWA needle into the right lower lung nodule (for Abstract no P67).

MWA was performed using a $15-\mathrm{cm}$ Emprint antenna with 75 watts for 1.53 minutes targeting $2.2 \mathrm{~cm}$ nodule of right lower lobe. The second session was executed 2 weeks after with $13-\mathrm{cm} \mathrm{Em-}$ print antenna, applying 75 watts for 1.20 minutes at left upper lobe nodule. There was no sign of pacemaker interference or malfunction post procedure.

Conclusion: MWA therapy can be safely performed in patients with pacemakers. Careful planning between Cardiologist and Interventional Pulmonologist is vital all throughout the process to assure ablation frequency do not affect pacemaker.

Disclosure: Nothing to disclose.

\section{P68 \\ Validation of a Portable Blood Gas Analyzer (EPOC Siemens) for Use in Challenging Field Conditions}

\section{J. Nawrocki ${ }^{1}$, M. Furian ${ }^{1}$, A. Buergin ${ }^{1}$, L. Mayer ${ }^{1}$, S. Schneider ${ }^{1}$, M. Mademilov ${ }^{2}$, M. Bloch', T.M. Sooronbaev ${ }^{2}$, S. Ulrich ${ }^{1}$, K.E. Bloch ${ }^{1}$ \\ ${ }^{1}$ University Hospital of Zurich, Department of Respiratory Medicine, Sleep Disorders Center, Zurich, Switzerland, \\ ${ }^{2}$ National Center for Cardiology and Internal Medicine, Bishkek, Kyrgyzstan}

Introduction: Mountain travellers and passenger of airplanes including patients with cardiopulmonary diseases may experience hypoxia-related illness. Portable arterial blood gas analyzers (pABGA) may serve as essential diagnostic tools in remote high altitude regions or during air travel but have not been extensively validated.
Methods: Accuracy of the novel, battery driven pABGA EPOC (Siemens Healthcare GmbH, Zurich, Switzerland) was compared to a validated stationary ABGA (Rapidpoint500, Siemens). In healthy individuals and patients with chronic obstructive pulmonary disease participating in trials of acetazolamide for prevention of altitude illness, arterial blood samples were obtained and analyzed by EPOC and Rapidpoint500. Bias (mean difference) and limits of agreement ( $95 \%$ confidence interval of bias) in paired measurements of the same blood samples by both devices were computed.

Results: In 105 individuals, 248 arterial blood samples were obtained, 108 at $760 \mathrm{~m}$ and 140 at 3,100 $\mathrm{m}$ in a remote Kyrgyz mountain area. Ranges of values measured by EPOC were: $\mathrm{pH}$ 7.241 to $7.473, \mathrm{PaCO}_{2} 2.87$ to $7.00 \mathrm{kPa}, \mathrm{PaO}_{2} 6.1$ to $14.3 \mathrm{kPa}$. Bias (95\% CI) between EPOC and RP500 were: $\mathrm{pH} 0.007$ (-0.029 to $0.044), \mathrm{PaCO}_{2}-0.04 \mathrm{kPa}(-0.64$ to 0.56$)$ and $\mathrm{PaO}_{2}-0.29 \mathrm{kPa}(-1.21$ to 0.62 ). A trend for overestimation of $\mathrm{pH}$ by EPOC in the low and underestimation in the high range could be corrected by the equation $\mathrm{pH} \_\mathrm{RP} 500=-1.37+\mathrm{pH}$ _EPOC* $1.19\left(\mathrm{R}^{2}=0.906\right)$, corrected bias -0.007 ( -0.023 to 0.009$)$. Handling of EPOC was easy and the device worked reliably.

Conclusions: The accuracy of blood gas analysis by the portable EPOC device in comparison to a standard stationary device was adequate for clinical use. Because of its portability and ease of handling, EPOC is a highly valuable diagnostic tool for use in everyday practice as well as and under challenging field conditions at low and high altitude.

Disclosure: Grants: Siemens Healthcare provided the devices, Swiss National Science Foundation, Lunge Zurich.

\section{P69 \\ OxyFrame: Validation of an Ear Pulse Oximeter for Integration into Eye Glasses Frames}

\author{
S.A. Guler ${ }^{1}$, F. Braun ${ }^{2}$, J. Solà', C. Verjus ${ }^{2}$, M. Marienfeld ${ }^{3}$, \\ M. Funke-Chambour ${ }^{1}$, J. Krauss ${ }^{2}$, T. Geiser $^{1}$ \\ ${ }^{1}$ Department of Pulmonary Medicine, University Hospital \\ Bern (Inselspital), Bern, ${ }^{2}$ Centre Suisse d'Electronique et de \\ Microtechnique (CSEM), Neuchâtel, ${ }^{3}$ Markus Marienfeld AG, \\ Ried-Brig, Switzerland
}

Introduction: Current oxygen delivery modes can be cumbersome for patients with chronic respiratory diseases who maintain an active lifestyle. Integrating nasal oxygen cannulas and a pulse oximeter into daily use eye glasses would reduce the burden of current solutions and would enable automated delivery of oxygen by a closed-loop system. With this study we validated an ear pavilion pulse oximeter specifically for integration into eye glasses frames.

Methods: We validated the OxyFrame pulse oximeter first on healthy volunteers undergoing normobaric hypoxia (AltiTrain$\mathrm{er}^{\circledR}$ ), and second on hypoxemic patients with chronic respiratory diseases. Participants completed a prespecified protocol simulating daily activities. We calculated correlation, error, and accuracy root mean square error $\left(\mathrm{A}_{\mathrm{RMS}}\right)$ to compare $\mathrm{S}_{\mathrm{p}} \mathrm{O}_{2}$ measured by OxyFrame, the standard sensor (Masimo ${ }^{\mathbb{R}}$ ) and arterial blood gas analysis (aBGA). 


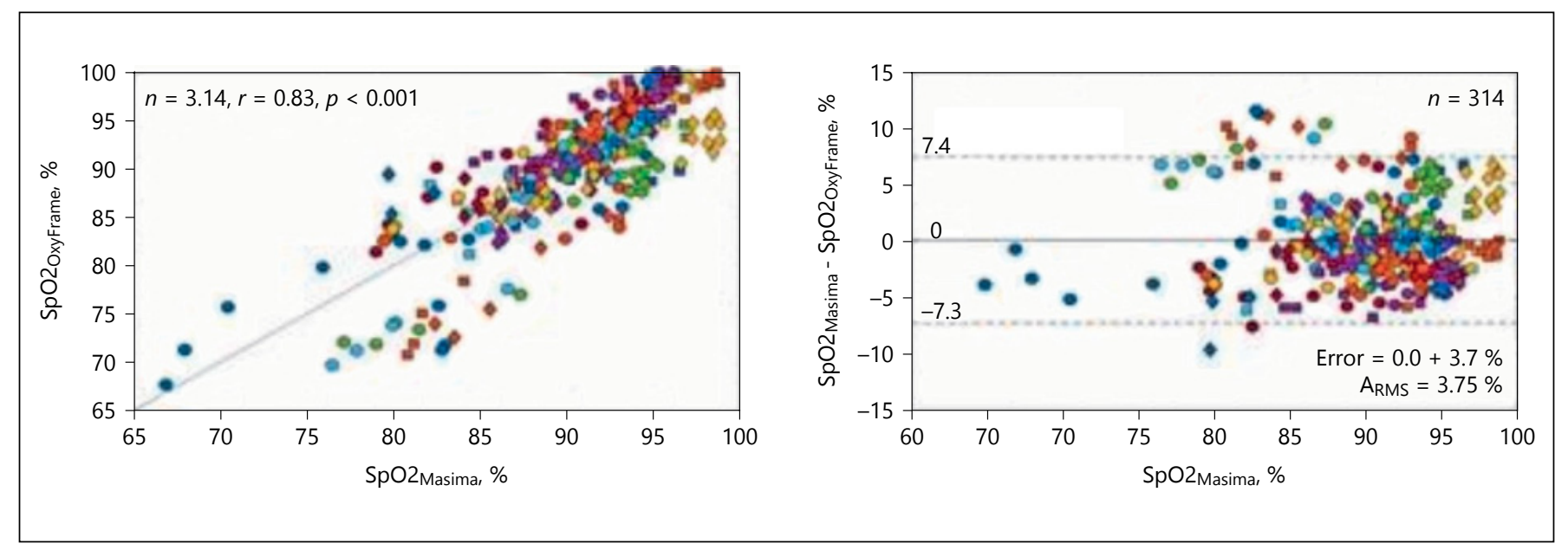

Fig. 1. (for Abstract no P69).

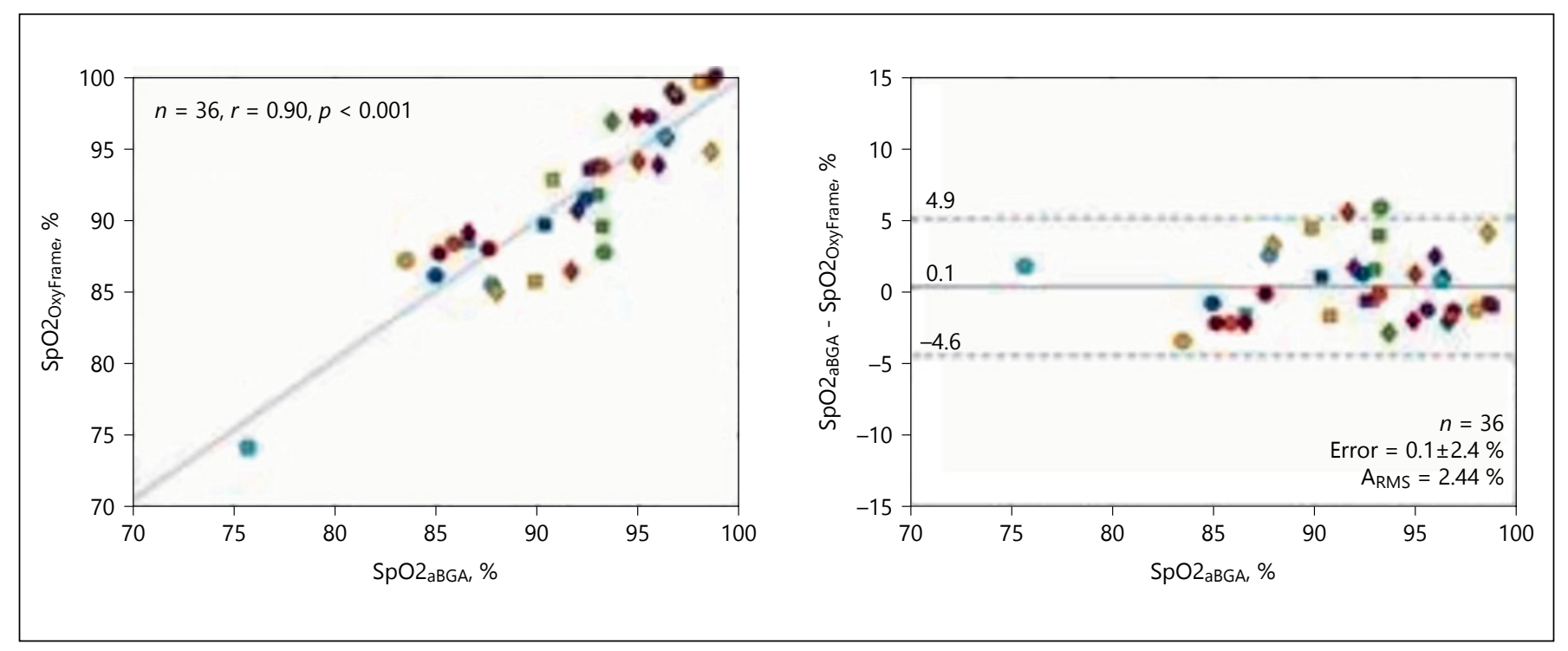

Fig. 2. (for Abstract no P69).

Results: Eight healthy volunteers provided $187 \mathrm{~S}_{\mathrm{p}} \mathrm{O}_{2}$ measurements. $\mathrm{S}_{\mathrm{p}} \mathrm{O}_{2}$ measured by OxyFrame and the standard sensor correlated strongly, with low error and high accuracy in healthy volunteers $\left(\mathrm{r}=0.84\right.$, error $\left.0.1 \pm 3.1 \%, \mathrm{~A}_{\mathrm{RMS}}=3.09 \%\right)$. One patient had to be excluded from analysis due to data storage issues, the remaining 19 patients provided 314 measurements. Performance characteristics of OxyFrame were similar when tested on patients $\left(\mathrm{r}=0.83\right.$, error $0.0 \pm 3.7 \%, \mathrm{~A}_{\mathrm{RMS}}=3.75 \%$ for comparison against the standard sensor, Figure 1 ; and $r=0.90$, error $0.1 \pm 2.4 \%$, and $\mathrm{A}_{\mathrm{RMS}}=2.44 \%$ for comparison against aBGA, Figure 2).

Discussion: We successfully validated the OxyFrame ear pavilion pulse oximeter and demonstrated highly accurate and reliable $\mathrm{S}_{\mathrm{p}} \mathrm{O}_{2}$ measurements meeting the requirements of ISO 80601-2-
61:2011 and the requirements for integration into a closed-loop oxygen delivery device.

Conclusion: This crucial step of the OxyFrame project enables further development of an automated closed-loop oxygen delivery system intended to provide a safer, more cost-effective and socially acceptable mode of oxygen delivery for patients with chronic hypoxemia.

Disclosure: This study was supported by the Lungenliga Bern. 


\section{P70 \\ Thoracoscopic Segmentectomy on ECMO to Manage a Second Primary Lung Cancer in a Patient with a Prior Left Pneumonectomy}

E. Koliakos ${ }^{1}$, I. Rotas' ${ }^{1}$ S. Colombier ${ }^{2}$, J.C. Grange ${ }^{3}$, D. Delay ${ }^{2}$, G. Gex ${ }^{4}$, J.Y. Perentes ${ }^{1,5}$

${ }^{1}$ Hôpital de Sion, Division of Thoracic Surgery, ${ }^{2}$ Hôpital de Sion, Division of Cardiothoracic Surgery, ${ }^{3}$ Hôpital de Sion, Division of Anesthesiology, ${ }^{4}$ Hôpital de Sion, Division of Pneumonology, Centre Hospitalier Du Valais Romand, Sion, ${ }^{5}$ Division of Thoracic surgery, Centre Hospitalier Universitaire Vaudois (CHUV),

Lausanne, Switzerland

Background: Second primary lung cancer management in patients who underwent prior lung resection is challenging because of the limited pulmonary reserve. In a curative intent, surgery or stereotactic radiation should be considered. Here we report the management of a pneumonectomized patient who developed a second primary lung cancer in his remaining lung.

Methods: Our patient had a prior history of central NSCLC stage IIIB that had been managed by neoadjuvant chemotherapy, a left pneumonectomy and adjuvant mediastinal radiation therapy. Six years later, the patient developed a new NSCLC lesion in the middle lobe (cT1N0M0). Stereotactic radiation was considered too risky because of the toxic cumulative radiation dose to the heart. Pulmonary functions (VEMS: 59\% and DLCO: 73\% predicted) and the cardiovascular evaluation were normal. Following multidisciplinary tumor board, the patient was considered for thoracoscopic middle lobe lateral segmentectomy on VV ECMO support.

Results: A jugulo-femoral VV ECMO was placed $(22 \mathrm{~F}$ jugular and $25 \mathrm{~F}$ femoral venous cannulas) following intubation. At full flow $(4.8 \mathrm{~L} / \mathrm{min})$, patient ventilation was interrupted. A VATS lateral segmentecomy of the middle lobe was performed. During the procedure, patient oxygenation and hemodynamics were stable. At the end of the case, VV ECMO was weaned, the patient was extubated and kept 48 hours in the ICU for surveillance. Chest tube was removed on POD1. The patient was discharged on POD5 and is doing well with no current recurrence. The histopathological analysis revealed a moderately differentiated NSCLC that was completely resected with no lymph node invasion (pT1N0L0V0Pn0R0).

Conclusion: The management of second primary lung cancer is challenging and should be tailored to each individual case. Extracorporeal life support has helped push the boundaries of patient operability.

Disclosure: Nothing to disclose.

\section{P71 \\ Postoperative Lingula Segment Torsion After Multi-Segmentectomy: A Case Report}

G. Somaini ${ }^{1}$, R. Bumm 1 , T. Latshang ${ }^{2}$, M. Furrer ${ }^{1}$

${ }^{1}$ Kantonsspital Graubünden, Thoracic Surgery, ${ }^{2}$ Kantonsspital

Graubünden, Pneumology, Chur, Switzerland

Aim: Although torsions of remaining lobes after lobectomy are rare complications, they classicly occurr as middle lobe torsions after right sided upper lobectomy. With the rise of sublobar lung resection techniques, atelectasis or even infarction of single remaining lung segments are more frequently observed. An individual clinical case and the diagnostic and therapeutic consequences are presented.

Method: A 62 year old female patient was submitted for surgery with a pulmonary mass in the left upper lobe, highly suspicious of a metastasis with a known primary invasive breast cancer. The patient underwent anatomical segment resection of segments one to three without complications. However, after five days an atelectasis of the lingula segment was radiologically diagnosed. Bronchoscopy showed a torsion of the lingula segment and a lack of ventilation.

Results: Re-thoracoscopy was performed, showing a completely atelectatic and twisted lingula segment. The segment was easily derotated and fixated with PDS sutures to prevent further torsion. After re-thoracoscopy, the postoperative course was uneventful, the $\mathrm{x}$-rays showed no atelectasis and bronchoscopy was normal.

Conclusion: While postoperative atelectasis after lung surgery is a common and often self-limiting problem, torsions of remaining segments are potentially dangerous complications that have to be recognized early by critical clinical assessment. Bronchoscopy is confirming the suspected diagnosis and immediate repair can be performed by re-thoracoscopy.

Disclosure: Nothing to disclose. 

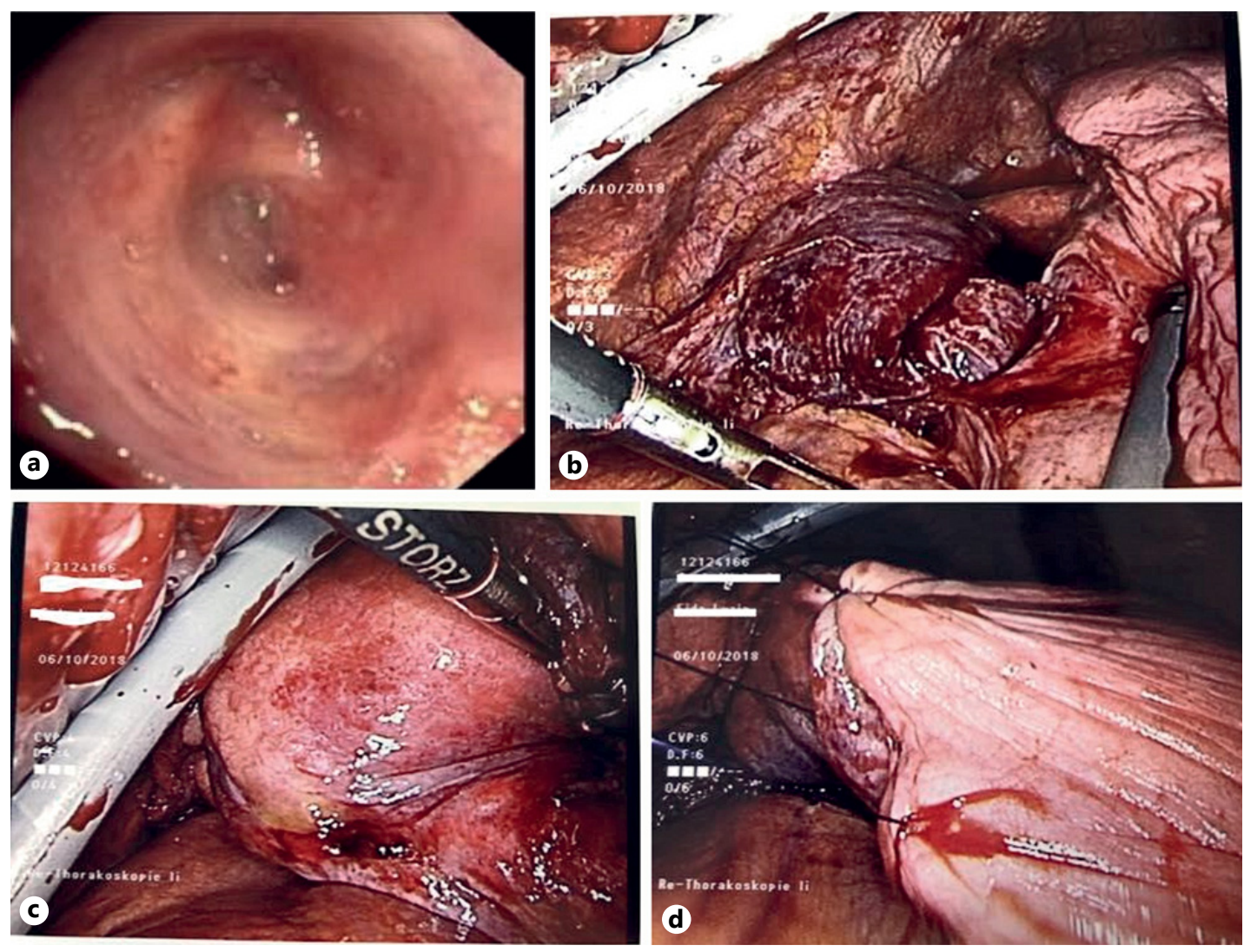

Fig. 1. a Bronchoscopy. b Thoracoscopy with atelectatic lingula on the left. c With de-rotated lingula and ventilation. d With fixation of lingula with PDS (for Abstract no P71).

\section{P72 \\ Anastomosing Hemangioma of the Posterior Mediastinum: A Case Report and Review of the Literature}

\section{S. Federici ${ }^{1}$, I. Letovanec ${ }^{2}$, H. Bouchaab ${ }^{3}$, H.B. Ris $^{1}$}

${ }^{1}$ Division of Thoracic Surgery, ${ }^{2}$ Institute of Pathology, ${ }^{3}$ Department of Oncology, Centre Hospitalier Universitaire Vaudois (CHUV), Lausanne, Switzerland

Background: Anastomosing hemangioma $(\mathrm{AH})$ is a benign vascular tumor originally described for the first time in the genitourinary tract in 2009. Rare cases of AH have also been described in the liver and in extra-genitourinary sites, with all cases were situated below the diaphragm.

We herein present to our knowledge the first case of supradiaphragmatica $\mathrm{AH}$, situated in the posterior mediastinum.

Method: A 52-years old woman underwent MRI examination for a persistent right-sided posttraumatic plexopathy. CT and MRI revealed an asymptomatic $2.0 \times 2.5 \mathrm{~cm}$ cystic lesion situated in the posterior mediastinum.

An excisional biopsy was performed with complete excision of the lesion by a left-side thoracoscopic approach. The patient was discharged on postoperative day 3 .
Macroscopic examination revealed a well-circumscribed cyst of $2.5 \times 2.0 \times 2.0 \mathrm{~cm}$ in size. The histological analysis showed a proliferation of capillary-sized vessels with multiples anastomoses and expression of endothelial (CD31, CD34) markers.

Results: $\mathrm{AH}$ is a rare subtype of hemangioma characterized by anastomosing vessel proliferations. John and Folpe, reported a series of $17 \mathrm{AH}$ cases observed in unusual locations other than the renal and hepatic sites but all of them were situated infradiaphragmatically except one (upper extremity). Most patients are in their $4^{\text {th }}-5^{\text {th }}$ decades with a slight male predilection. Most of the lesions are unilateral and the size varied from 0.1 $\mathrm{cm}$ to $8 \mathrm{~cm}$.

Clinical and imaging presentation evoked a differential diagnosis including sarcoma, neurogenic tumors, lymphoma, bronchogenic cyst, pulmonary sequestration and aortic disease. Most reports confirm the benign behavior of $\mathrm{AH}$, however, most of them were treated by a complete excision because malignant disease could not be a priori excluded.

Conclusion: We reported for the first time a case of anastomosing hemangioma situated in the posterior mediastinum which demonstrated the typical clinical, radiological features of an $\mathrm{AH}$, despite its unusual localization.

Disclosure: Nothing to disclose. 


\section{Oral Sessions}

\section{Thoracic Surgery Session 4: Free Communications}

\section{1 \\ Outcomes of Patients Discharged Home with a Chest Tube Following Anatomical Lung Resection: A Multicenter Cohort Study}

\author{
F. Minervini ${ }^{1}$, W. Hanna ${ }^{2}$, A. Brunelli ${ }^{3}$, F. Farrokhyar ${ }^{2}$, T. Miyazaki ${ }^{3}$, \\ L. Bertolaccini ${ }^{4}$, M. Scarci ${ }^{5}$, J. Agzarian ${ }^{2}$, M. Coret ${ }^{2}$, K. Hughes ${ }^{2}$, \\ Y. Lopez-Hernandez ${ }^{2}$, L. Schneider ${ }^{2}$, C. Finley ${ }^{2}$, Y. Shargall ${ }^{2}$
}

${ }^{1}$ Thoracic Surgery, Luzerner Kantonsspital, Lucerne, Switzerland, ${ }^{2}$ McMaster University, Hamilton, ON, Canada, ${ }^{3} \mathrm{St}$ James University Hospital, Leeds, United Kingdom, ${ }^{4}$ Maggiore Teaching Hospital, Bologna, ${ }^{5}$ San Gerardo Hospital, Monza, Italy

Introduction: Prolonged air leak following lung resections remains a common postoperative complication. We evaluated the outcomes of those patients and potential risk factors associated with adverse outcomes.

Methods: Retrospective analysis of prospectively collected data from four academic centers between 1.2014 and 12.2017. Data was analyzed for 253 patients, representing $9.0 \%$ of all patients undergoing anatomical lung resections during that period. Chi-square and Mann-Whitney $U$ tests were used to asses for patients and operative parameters associated with outcomes post discharge. Logistic regression was performed to evaluate factors associated with risk of empyema development and need for readmissions and intervention.

Results: Of 253 patients analyzed, there were $67 / 857$ patients from center A (7.8\%), 30/759 from center B (3.95\%), 147/931 from center C (15.78\%), and 9/247 from center D $(3.64 \%)(\mathrm{p}<$ $0.001)$. Median age was $69(19-88), 56 \%$ males. Overall, 49 patients $(19.4 \%)$ were readmitted $(21 \%, 0 \%, 23 \%, 11 \%$, centers A-D, respectively, $\mathrm{p}=0.029)$ and analyzed. Of those, $18(37 \%)$ developed empyema, $11(22 \%)$ required surgery and $3(6 \%)$ died. Median LOS was 8 (3-63) and 7 (3-30) days for readmitted vs not-readmitted patients $(\mathrm{p}=0.588)$. Comorbidities $(\mathrm{p}=0.1-0.9)$, approach (MIS vs thoracotomy, $p=0.75)$ and extent of resection $(p=0.577)$ were not associated with risk of readmissions. Median overall initial duration of chest tube was 22 days (4-141) for readmitted patients vs $16(1-148)$ days for not readmitted ( $\mathrm{p}<0.001)$. Duration of chest tube stay was the only factor associated with development of empyema ( $\mathrm{p}=0.003)$. The risk of empyema increased 3 -fold $(\mathrm{OR}=2.94)$ when chest tube was left in-situ for more than 20 days.

Conclusion: Home discharge with a chest tube following lung resection is associated with significant adverse events. Given high risk of empyema development, removal of chest tube should be considered, when appropriate, after 20 days. Our data suggests potential need for active post-discharge outpatients program, in order to diminish subsequent risk of morbidity and mortality.

Disclosure: Nothing to disclose.

\section{2 \\ The Impact of Patient Compliance and Individual Components in an ERAS Pathway on the Outcome of Anatomical VATS Resections}

\author{
C. Forster $^{1}$, V. Doucet ${ }^{1}$, J.Y. Perentes ${ }^{1}$, E. Abdelnour-Berchtold ${ }^{1}$, \\ T. Krueger ${ }^{1}$, L. Rosner ${ }^{2}$, M. Gonzalez ${ }^{1}$ \\ ${ }^{1}$ Thoracic Surgery, ${ }^{2}$ Anesthesiology, Lausanne University \\ Hospital, Lausanne, Switzerland
}

Introduction: Implementation of an enhanced recovery after surgery (ERAS) pathway has shown to improve post-operative outcome. However, the impact of compliance (overall and to specific elements of the program) has been rarely reported in thoracic surgery and was the subject of this study.

Methods: We included all consecutive patients undergoing video-assisted thoracoscopy (VATS) anatomical pulmonary resection after the implementation of an ERAS pathway. Demographics, surgical characteristics and peri- and post-operative adherence to 16 elements of the ERAS program were assessed. Post-operative outcomes and length of stay were compared between low $(<75 \%$ of adherence) and high compliance $(>75 \%)$ groups. Logistic regression was undertaken to identify individual elements related to post-operative complications and a length of stay of $>4$ days (delayed discharge).

Results: A total of 192 ERAS patients, with a median age of 66 years (range 28-87), underwent VATS anatomic lung resection (109 lobectomies, 84 segmentectomies). There was no 30-day mortality and the re-operation rate was $6.3 \%$. The overall compliance to ERAS protocol was $76 \%$. Overall and pulmonary postoperative complications were observed in $33 \%$ and $28 \%$, respectively. The median postoperative length of hospitalization was 4 days (range 1-100). The high-compliance group was correlated with fewer complications (OR: $0.25, \mathrm{p}<0.0001$ ) and lower rate of delayed discharge (OR: $0.39, \mathrm{p}=0.0013)$. Early removal of chest tubes (OR: $0.26, \mathrm{p}<0.002)$, use of electronical drainage (OR: 0.39, $\mathrm{p}=0.036$ ), cessation of opioid administration on day 3 (OR: $0.28, p=0.016$ ) and early feeding (OR: $0.12, \mathrm{p}=0.014$ ) were all associated with reduced postoperative complications. Shorter hospital stay was correlated with early removal of chest tubes (OR: $0.12, \mathrm{p}<0.0001)$ and cessation of opioid administration on day 3 (OR: $0.23, \mathrm{p}=0.001$ ).

Conclusion: High compliance to ERAS program (overall and to some individual elements) seems to improve the post-operative outcome in patients undergoing anatomical pulmonary VATS resections.

Disclosure: Nothing to disclose. 


\section{3 \\ Uniportal Thoracoscopic Anatomic Segmentectomy: Clinical and Oncological Outcome of 150 Cases}

\section{K. Gioutsos, J.A. Lutz, P. Dorn, R.A. Schmid, G. Kocher}

Division of General Thoracic Surgery, Inselspital, Bern University Hospital, Switzerland, Bern, Switzerland

Introduction: Pulmonary segmentectomies using minimally invasive techniques are increasingly performed. Especially in patients with limited pulmonary reserve or comorbidities, VATS and in particular uniportal VATS results in favorable short-term outcomes with less postoperative complications, shorter hospital stay and faster recovery of pulmonary function. The aim of this study was the evaluation of clinical short-term outcomes as well as of oncological long-term outcomes.

Methods: Retrospective analysis of all patients that underwent single-port anatomical segmentectomy from $03 / 2015$ to $12 / 2017$ at our institution. For the oncological follow-up, data until the end of December 2018 were taken into consideration.

Results: A total of 150 consecutive patients were included with diagnoses of lung cancer $(n=96)$, metastasis $(n=30)$ and benign lesions $(n=26)$. Besides resection of one single lung segment $(n=$ 86), 39 bi-segmentectomies and 21 tri-segmentectomies were performed. 1 conversion to thoracotomy was necessary. Primary lung tumour types were adenocarcinoma $(n=65)$, squamous cell carcinoma $(\mathrm{n}=18)$, neuro-endocrine tumors $(\mathrm{n}=8)$ and SCLC $(\mathrm{n}=5)$.

Mean tumor size was $19.1 \mathrm{~mm}$. All patients underwent systematic regional and mediastinal lymph node dissection. Mean number of resected lymph nodes was $12.55 \pm 8.06$ in $3.85 \pm 1.89$ lymph node stations. Seven patients (7.3\%) were nodally upstaged. The 30 -day mortality and morbidity rates were $0.66 \%$ and $11.3 \%$, respectively. Main postoperative complications were pneumonia $(n=7)$ and prolonged air leak $(n=7)$. The median duration of hospital stay was 3 days. In the median follow-up time of 25 months (IQR 21-30), 3 patients showed local recurrence and 2 patients presented with metachronous NSCLC. 8 non-NSCLC related deaths and 2 deaths due to metastasis of NSCLC without signs of local recurrence occurred.

Conclusion: Uniportal thoracoscopic anatomical segmentectomy is a safe and effective surgical alternative to traditional lung resections. Short-term clinical outcomes are promising and the long-term oncological outcome remains to be further assessed in the years to come.

Disclosure: Nothing to disclose.

\section{4 \\ Chest Wall Resections and Reconstructions for Malignant Diseases: A Single Center Experience in 158 Cases}

L.Elahi-Rausis ${ }^{1}$, E. Abdelnour ${ }^{1}$, M. Gonzalez ${ }^{1}$, D. Hasselbach ${ }^{2}$,
N. Leslie ${ }^{3}$, T. Krueger
, W. Raffoul ${ }^{2}$, H.-B. Ris

${ }^{1}$ Thoracic Surgery, ${ }^{2}$ Plastic and Reconstructive Surgery, ${ }^{3}$ Pneumonology, Centre Hospitalier Universitaire Vaudois (CHUV), Lausanne, Switzerland

Introduction: The surgical management of tumors involving the chest wall is challenging and should achieve complete resection and insure stability/function. Here we reviewed 158 chest wall resections performed in one center for diverse malignancies over a 15-year period and report in-hospital morbidity/mortality and oncological outcome.

Methods: The records of 158 chest wall reconstructions performed in 150 patients between 2003 and 2018 by a joint team of reconstructive and thoracic surgeons were reviewed. We determined, for each patient, the extent of resection, the reconstruction type, the postoperative surgical and non-surgical complications and the oncological long-term outcome.

Results: Chest wall resections were performed in the context of lung cancer $(31 \%)$, primary chest wall tumors $(33 \%)$, breast cancer $(15 \%)$ or metastasis $(20 \%)$. Full thickness (skin, muscle, ribs \pm sternum \pm clavicule) chest wall resection was performed in 66 cases while the other 92 cases involved bony chest wall with no superficial soft tissue. Associated lung resections were required in 72 patients (wedge $43 \%$, lobectomy $43 \%$, pneumonectomy $14 \%$ ). Chest wall reconstructions were performed using pedicled muscle flaps (15\%) prosthetic chest wall subsitutes (mesh with and without methyl methacrylate reinforcement, $38 \%$ ) or a combination of both (47\%). The postoperative mortality was of $2.5 \%$ and related to septic/hemorrhagic shock $(\mathrm{n}=3)$ and pulmonary embolism $(\mathrm{n}=$ 1). Postoperative morbidity consisted of pneumonia (21\%), arrhythmia (10\%), pleural effusion (15\%) and pulmonary embolism $(2 \%)$. A complete tumor resection was obtained in 123 patients (78\%) and chest wall stability and integrity was achieved in all patients. Mean patient follow-up was of $44 \pm 43$ months Overall and disease free survival were $46 \pm 44$ and $31 \pm 41$ months, respectively.

Conclusion: Chest wall resections and reconstructions performed in the context of a multimodal approach can offer rewarding overall and disease free survivals while preserving the chest wall integrity and functionality. Postoperative morbidity and mortality rates are acceptable.

Disclosure: Nothing to disclose. 


\section{5 \\ Outcome of Incidental and Secondary Bronchogenic Carcinoma Following Lung Transplantation}

\section{Forster ${ }^{1}$, A. Koutsokera ${ }^{2}$, J.-D. Aubert ${ }^{2}$, M. Gonzalez ${ }^{1}$, T. Krueger ${ }^{1}$,} J.-Y. Perentes ${ }^{1}$, E. Abdelnour-Berchtold ${ }^{1}$

${ }^{1}$ Thoracic Surgery, ${ }^{2}$ Pneumology, Lausanne University Hospital, Lausanne, Switzerland

Introduction: International registries report an overall incidence of $4 \%$ of lung cancer in recipients after lung transplantation. Most concern single lung transplant with past smoking history, idiopathic pulmonary fibrosis or COPD. These patients with poor prognosis develop malignancy in their remaining native lungs. However, bronchogenic carcinoma in the transplanted allograft is rare and little is known about double-lung transplant series paired to their donor lung characteristics.

Methods: A retrospective single institution experience based on all patients undergoing lung transplantation between January 2004 and December 2018. All recipients with (group 2) and without (group 1) lung cancer were identified. Two subgroups were then defined according to unforeseen tumors discovered on the explanted lungs (group 2a) or to those that developed de novo on the allograft (group 2b). Kaplan-Meier curves, demographic and pairwise tests were computed depending on the dataset.

Results: From all 257 patients, $95 \%(\mathrm{n}=244)$ underwent a double-lung transplant procedure. Out of the 16 patients diagnosed with lung cancer, nine were incidental findings on the explanted lung (group 2a) and seven had secondary malignancy (group 2b). Survival was significantly lower in group $2 b(\mathrm{p}<0.05)$ (Figure 1 ) and mean time interval between transplant and diagnosis was 65.3 months. Mean age of donors was $48.2 \pm 16$ years in group 1 and $60.4 \pm 6.8$ in group $2 b(p<0.001)$. The donor's smoking history was similar in both groups. Mean age of recipients at transplantation was $47.9 \pm 14$ years in group $1,55.7 \pm 6.7$ in group $2 \mathrm{a}(\mathrm{p}<0.05)$ and $61 \pm 4.2$ in group $2 b(\mathrm{p}<0.05)$.

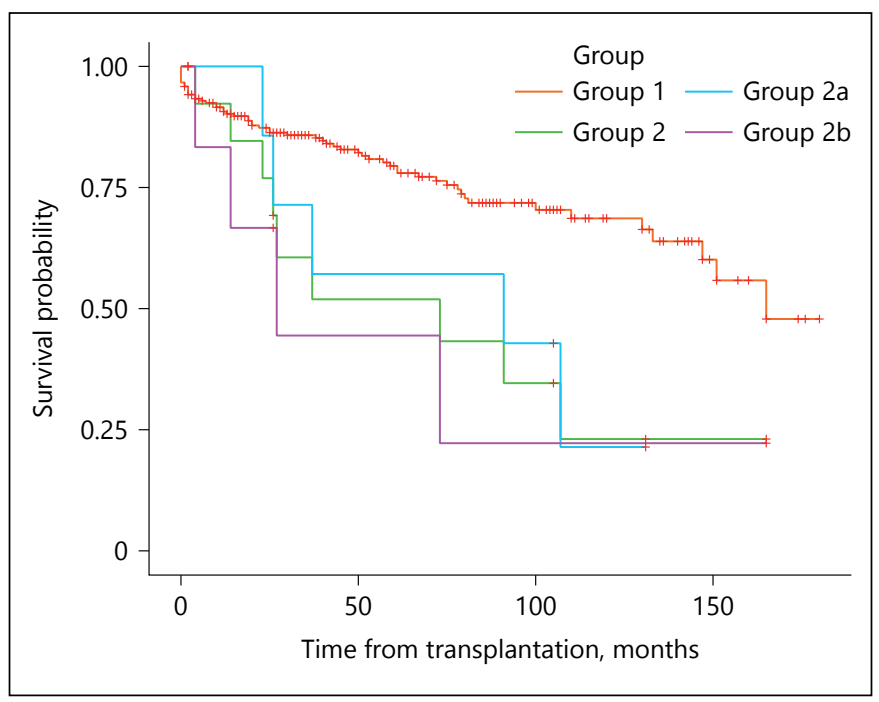

Fig. 1. (for Abstract no O05).
Conclusion: We conclude that the age of both donors and recipients is a risk factor for lung cancer in transplant recipients whereas neither the donor's smoking status nor the recipient's primary diagnosis is associated with this complication. Overall, a diagnosis of lung cancer has a poor prognosis in this population.

Disclosure: Nothing to disclose.

\section{6 \\ Clinical Outcome of Loculated Parapneumonic Effusions Managed Surgically or by Intrapleural Fibrinolysis: A Comparative Multicenter Study}

\author{
$\underline{\text { S. Federici }}{ }^{1}$, B. Bedat ${ }^{2}$, E. Abdelnour ${ }^{1}$, S. Dackam ${ }^{2}$, J. Hayau ${ }^{1}$, \\ M. Gonzalez ${ }^{1}$, T. Krueger ${ }^{1}$, L. Noirez ${ }^{3}$, F. Triponez ${ }^{2}$, W. Karenovic ${ }^{2}$, \\ J.Y. Perentes ${ }^{1}$ \\ 'Thoracic Surgery, Centre Hospitalier Universitaire Vaudois \\ (CHUV), Lausanne, ${ }^{2}$ Thoracic Surgery, Hopitaux Universitaires \\ de Geneve, Geneve, ${ }^{3}$ Pneumonology, Centre Hospitalier \\ Universitaire Vaudois (CHUV), Lausanne, Switzerland
}

Introduction: The management of loculated parapneumonic effusions is debated and includes non-surgical (drainage + fibrinolysis) and surgical approaches (VATS/Thoracotomy). Here we present the outcome of patients with parapneumonic empyema managed over 4 years in two institutions, one with an early surgical and the other with a non-surgical approach.

Methods: The charts of patients with loculated parapneumonic effusions managed in both institutions between January 2014 and December 2017 were reviewed. Patients with persisting parapneumonic loculated pleural effusions on CT-scan despite chest tube drainage were managed preferentially by early surgery in one center (group I) and by fibrinolysis in the other (group II). For each patient, we recorded the age, gender, hospital stay, morbidity, mortality, infection control and pleural opacity decrease on chest $\mathrm{X}$-ray before and 7 days after treatment.

Results: Groups I $(\mathrm{n}=88)$ and II $(\mathrm{n}=78)$ did not differ regarding patient age (57 \pm 16 vs $60 \pm 17$ years) and gender (70\% vs $70 \%$ male). There was no 30-day mortality in both groups and no significant difference in morbidity with respect to hemothoraces (4.6\% vs $8 \%)$ although significantly more arrhythmias were observed in group I compared to II $(6 \%$ vs $0 \%, \mathrm{p}<0.05))$. In group I, 55 patients $(62 \%)$ could be managed by VATS. In group II, 15 patients $(20 \%)$ required an additional drainage while 12 patients (16\%) had to undergo surgical decortication. The duration of chest tube drainage (4 [1-29] vs $6[1-34]$ days, $\mathrm{p}<0.05)$ and hospital stay $(12$ [3-157] vs 18 [4-373] days, $p<0.05)$ were significantly lower in the group I but infection control (100\%) and pleural opacity improvement were comparable in both groups (opacity decrease by $-24 \pm 19 \%$ and $-28 \pm 19 \%$ in groups I and II respectively).

Conclusion: Surgically managed complicated parapneumonic effusions had a shorter hospitalization and chest tube drainage but overall clinical and radiological outcome was similar for surgical and non-surgical approaches.

Disclosure: Nothing to disclose. 


\section{Pediatric Pulmonary Scientific Session 1: Free Communications}

\section{7 \\ The Skin Microbiome Drives Immune Maturation and Exacerbation of Both Skin and Airway Inflammation}

N. Ubags ${ }^{1}$, A. Trompette ${ }^{1}$, B. Nibbering ${ }^{1}$, J. Pernot ${ }^{1}$, C. Pattaroni ${ }^{1,2}$, A. Rapin ${ }^{1}$, L. Nicod ${ }^{1}$, B. Marsland ${ }^{1,2}$

'Service de Pneumologie, CHUV, Lausanne, Switzerland, ${ }^{2}$ Department of Immunology and Pathology, Monash

University, Melbourne, VIC, Australia

Introduction: Skin allergen sensitization can induce atopic dermatitis, a potential starting point of the 'atopic march', which describes the developmental progression of atopic dermatitis in infancy, to allergic rhinitis and allergic asthma in childhood. We hypothesized that the skin microbiome drives immune maturation, and that the maturation state at the time of skin allergen sensitization determines the allergic airway inflammation phenotype following allergen challenge.

Methods: Neonatal and adult mice were subjected to a 3-week epicutaneous allergen sensitization protocol followed by intranasal allergen challenges. Skin and lung tissue inflammation was assessed using histological and flow cytometric analysis. In addition, the skin transcriptome and microbiome were analyzed using RNA-seq and $16 S$ rRNA sequencing respectively.

Results: The time point in life (early life vs. adulthood) at which the first sensitization takes place determines a Th2 vs. a mixed Th2/Th17 driven allergic inflammation in both the skin and the lung. Naïve neonatal mouse skin exhibited decreased frequencies of antigen presenting cells, antigen uptake and processing capacity compared to adults. Transcriptome analysis revealed that genes associated with immune and antibacterial responses were enriched in adult compared to neonatal skin. Similar genes were downregulated in adult germ-free mouse skin, suggesting an important role for the microbiome. Epicutaneous allergen sensitization in adult mice was characterized by increased Staphylococcus aureus abundance on the skin, and neutrophil accumulation and increased IL17 gene expression in both the skin and the lung.

Conclusion: Our experimental model indicates that the skin microbiome drives immune maturation and exacerbation of both skin and airway inflammation. Increased understanding of the effect of the skin microbiome on immune maturation in early life and its influence in the development of allergic diseases, may aid in the development of targeted therapies for the prevention of allergic asthma in atopic patients.

Disclosure: Nothing to disclose.

\section{8 \\ Exposure to Moderate Air Pollution and Associations with Lung Function at School-Age: A Birth Cohort Study}

\author{
F. Decrue ${ }^{1}$, J. Usemann ${ }^{1}$, I. Korten ${ }^{2}$, E. Proietti ${ }^{1}$, O. Gorlanova ${ }^{1}$, \\ D. Vienneau ${ }^{3}$, O. Fuchs ${ }^{2}$, P. Latzin' ${ }^{2}$, M. Röösli ${ }^{3}$, U. Frey' ${ }^{1}$, BILD Study \\ Group \\ 'University of Basel Children's Hospital (UKBB), Basel, \\ ${ }^{2}$ Inselspital, Bern University Hospital, Bern, ${ }^{3}$ Swiss Tropical and \\ Public Health Institute Basel, Basel, Switzerland
}

Introduction: Adverse effects of higher air pollution levels before and after birth on subsequent lung function are often reported in the literature. We assessed whether low-to-moderate levels of air pollution during preschool-age impact upon lung function at school-age.

Methods: In a prospective birth cohort of 304 healthy termborn infants, 232 (79\%) completed lung function at follow-up at six years. Levels of individual air pollution (nitrogen dioxide $\left(\mathrm{NO}_{2}\right)$ and ozone $\left(\mathrm{O}_{3}\right)$, particulate matter with a diameter $<10 \mu \mathrm{m}$ $\left.\left(\mathrm{PM}_{10}\right)\right)$ were estimated for the time windows pregnancy, first and sixth year, and birth until follow-up at six, using spatial-temporal models. Time window means were compared to the WHO guideline limits. Associations of exposure windows with spirometry and body-plethysmography indices were analysed using regression models, adjusting for potential confounders. For subgroup analysis, air pollution exposure was categorized into quartiles.

Results: Mean $\mathrm{NO}_{2}$ level from birth until follow-up was [mean (range)] [11.8 $\left(4.9\right.$ to $\left.35.9 \mu \mathrm{g} / \mathrm{m}^{3}\right)$ ], which is almost 4-times lower than the WHO suggested limit of $40 \mu \mathrm{g} / \mathrm{m}^{3}$. Increased air pollution levels from birth until follow-up were associated with reduced lung function. In the subgroup analysis, the 52 children exposed to $\mathrm{NO}_{2}$ levels from the highest quartile during pregnancy, first and second year of life and from birth until follow-up, had a significant decrease in $\mathrm{FEV}_{1}$. Per interquartile range increase of $\mathrm{NO}_{2}, \mathrm{FEV}_{1}$ decreased by [z-score change (95\% confidence interval)] $[-1.07$ $(-1.67$ to -0.47$)],[-1.02(-1.66$ to -0.39$)],[-0.51(-0.86$ to -0.17$)]$ and $[-0.80(-1.33$ to -0.27$)]$, respectively.

Conclusion: Our results suggest that exposure to higher $\mathrm{NO}_{2}$ levels, which are still much lower than WHO guideline limits, may be associated with reduced lung function at school-age. These findings support the concept of age and dose-dependent pollution effects on lung function in school-aged children and underline the importance of pollution reduction measures.

Disclosure: Nothing to disclose. 


\section{9}

\section{Pulmonary Late Effects After Hematopoietic Stem Cell Transplantation in Childhood Cancer Survivors in Switzerland}

\author{
M. Otth ${ }^{1}$, C. Schindera ${ }^{1}$, T. Güngör ${ }^{2}$, M. Ansari ${ }^{3}$, C. Kuehni ${ }^{1}$ \\ ${ }^{1}$ Institute of Social and Preventive Medicine, Bern, \\ ${ }^{2}$ Department of Pediatric Immunology and Blood and Marrow \\ Transplantation, Children's Hospital Zurich, University of Zurich, \\ Zurich, ${ }^{3}$ Department of Pediatrics, Oncology and Hematology \\ Unit, Geneva University Hospital, Geneva, Switzerland
}

Introduction: Around 300 children and adolescents are annually diagnosed with cancer before the age of 20 years in Switzerland. The 10 -year survival rate exceeds $85 \%$. This leads to increasing numbers of Childhood Cancer Survivors (CCS) and to a rise in long-term sequelae, as for pulmonary morbidity and mortality. CCS after hematopoietic stem cell transplantation (HSCT) are at particular risk. This observational study aims to describe self-reported pulmonary sequelae in this group.

Method: We included CCS diagnosed between 1976 and 2010 at age $0-16$ years, who survived $\geq 5$ years from diagnosis, and had been treated with autologous or allogeneic HSCT. We sent them a questionnaire with questions on pulmonary symptoms and diagnoses (e.g. fibrosis, emphysema, recurrent pneumonia, chronic cough). Medical data were gathered from the Swiss Childhood Cancer Registry (SCCR) and from medical records.

Results: We included 137 ( $52 \%$ male) CCS with a mean age of 7.4 years (range $0.1-17.6$ ) at diagnosis, 9.6 years $(0.6-23.4)$ at HSCT, and with a mean follow-up of 12 years (5.1-31.4). Fifty-six CCS had been treated with lung radiation, 44 with at least one pulmotoxic agent (Bleomycin, Busulfan, Nitrosureas), and 21 with thoracic surgery. Twenty-nine CCS (21\%) reported 39 pulmonary problems, of which the most common were recurrent pneumonia and chronic cough $(71 \%)$. The number of pulmonary problems did not differ between CCS treated with allogeneic $(n=71)$ or autologous $(n=63)$ HSCT.

Conclusion: One fifth of CCS after HSCT reported pulmonary problems. Fibrosis and emphysema, pulmonary long-term sequelae verifiable by pulmonary function tests, were reported rarely (7\%). This could be because subclinical lung disease remains long asymptomatic and thus does not result in a medical diagnosis. As a next step I will collect pulmonary function tests in this cohort to assess early stage disease.

Disclosure: Nothing to disclose.

\section{0 \\ CD90 Identifies a Distinct Pulmonary Mesenchymal Cell Subtype with Both Immune Modulatory and Perivascular-Like Function in Postnatal Human Lung}

$\underline{\text { S. Hall }}^{1,2}$, L. Wang ${ }^{1,2}$, P. Dorn' 1 , S. Zeinali ${ }^{3}$, S. Berezowska ${ }^{4}$, R.-W. Peng 1,2, T. Marti ${ }^{1,2}$, O. Guenat ${ }^{1,3}$, P. Bode ${ }^{5}$, U. Moehrlen ${ }^{6}$, R. Schmid ${ }^{1,2}$

'Department of General Thoracic Surgery, Inselspital, Bern University Hospital, ${ }^{2}$ BioMedical Research, ${ }^{3}$ ARTORG Center for Biomedical Engineering Research, ${ }^{4}$ Institute of Pathology, University of Bern, Bern, ${ }^{5}$ Department of Pathology and Molecular Pathology, University Hospital Zurich, ${ }^{6}$ Department of Pediatric Surgery, University Children's Hospital, Zurich, Zurich, Switzerland

Introduction: In human lung, both aging and pathological injury have an impact on not only epithelial but also mesenchymal lineages, yet little is known regarding specific lung mesenchymal cell populations impacted during these processes.

Methods: We used a combination of prospective cell-sorting strategies, immunofluorescence analysis and advanced cell culture technology to identify and characterize mesenchymal cell subtypes in postnatal human lung tissue obtained from patients undergoing elective surgery for congenital pulmonary airway malformation (CPAM) and other airway abnormalities.

Results: In unaffected postnatal human lung there was a 5 fold increase in the mesenchymal compared to the epithelial compartment. The mesenchymal compartment consists primarily of CD $90^{+}$cells enriched in niche factors CXCL12 and PDGFRa. Phenotypically, $\mathrm{CD} 90^{+}$cells express surface markers commonly found on mesenchymal stem/stromal cells, yet were limited in their mesenchymal differentiation capacity. Tissue sections showed CD90 $0^{+}$ cells in close proximity to uncommitted cuboidal EpCAM ${ }^{+} \mathrm{CD} 73^{+}$ cells in the alveolar region, suggestive of an alveolar unit. In culture, postnatal $\mathrm{CD} 0^{+}$mesenchymal cells readily internalize virosome particles with reconstituted influenza type A and type $\mathrm{B}$ viral envelopes, and were able to inhibit the activation of $\mathrm{CD}^{+}$ $\mathrm{T}$ cells from peripheral blood or lung in a dose- and cell contactdependent manner. Despite their declining numbers in adult human lung, there was a re-emergence in lung mesenchyme in lungs from patients with chronic obstructive pulmonary disease (COPD), coupled with an impairment in the ability of $\mathrm{CD} 90^{+}$mesenchymal cells to support microvessel formation. In tissue sections from CPAM patients and other pathologies, we observed cystic airspaces and dysplastic alveolar regions with an increase in $\mathrm{CD} 90^{+}$ and $\mathrm{CD} 90^{+} \mathrm{PDGFR}^{+}$mesenchymal cells.

Conclusion: Using postnatal and adult human lung, these data provide important new information regarding the immunophenotypic identity of key mesenchymal lineages and track their change in diverse setting of CPAM and other airway abnormalities including COPD.

Disclosure: Nothing to disclose. 


\section{Author Index}

Abdelnour, E. O04, O06

Abdelnour-Berchtold, E. O02, O05

Adhanom Sengal, A. P30

Adler, D. P07, P34, P38, P56

Aebi-Di Salvo, R. P53

Aeschbacher, S.S. P11

Agzarian, J. O01

Amrein, M.A. P61

Andenmatten, S. P02

Annaheim, S. P35

Ansari, M. O09

Aubert, J.-D. P03, P43, O05

Azzola, A. P28

Bader, P. P47

Bader, P.R. P10

Bailly, S. P38

Barazzone, C. P63

Barnowski, S. P51

Baty, F. P04, P09, P35, P64

Baumgartner, P. P19

Bayer Zubek, V. P29

Bedat, B. P07, P56, O06

Bédat, B. P50

Beghetti, M. P44

Beigelman, C. P40, P55

Belevsky, A. P29

Berezowska, S. P61, O10

Berlier, C. P45, P47, P49

Bernasconi, E. P03

Bertelli, C. P01

Bertolaccini, L. O01

Beuschlein, F. P22, P33

Beyeler, S. P30

Bezel, P. P31

Blanchon, S. P63

Blank, F. P30

Bloch, K. P18, P47

Bloch, K.E. P10, P11, P13,

P15, P16, P25, P26, P68

Bloch, M. P68

Blouin, J.-L. P54

Blundell, T.L. P05

Bochud, P.-Y. P40

Bode, P. O10

Boeck, L. P05

Boesch, M. P09, P35

Boss, A. P21

Bouchaab, H. P72

Bovet, L. P06

Brack, T. P27

Braun, F. P69
Bremerich, J. P59

Bridevaux, P.-O. P28

Brun, P. P06

Brunel, A.-S. P40

Brunelli, A. O01

Brutsche, M. P04, P27, P35, P64

Brutsche, M.H. P09

Bryant, J. P05

Buergin, A. P10, P13, P15, P16, P25, P26, P68

Bührer, E. P61

Bumm, R. P71

Burbaud, S. P05

Camenzind, M. P35

Cantero, C. P34

Casaulta, C. P54

Casutt, A. P40

Charbonnier, F. P28

Clarenbach, C. P28

Clarenbach, C.F. P19, P27

Colombier, S. P70

Corbaz, A. P41

Coret, M. O01

Couchepin, J. P40

Crowe, L. P44

Daccord, C. P52, P53, P55

Dackam, S. O06

Decrue, F. $\mathrm{O} 08$

Delay, D. P70

Deng, H. P57

Despland, C. P65

Diamanti, E. P43

Diedrich, J. P29

Dionne, M. P05

Dorn, P. P57, O03, O10

Doucet, V. O02

Duchna, H.-W. P28

Dumont, P. P53

Ebener, S. P51

Egger, B. P55

Elahi-Rausis, L. O04

Emilov, B. P13, P15, P16, P25, P26

Eng, P. P53

Estebesova, B. P13, P15, P16, P25, P26

Esteves Carreira, A.L. P41

Fang, L. P09, P23
Farrokhyar, F. O01

Federici, S. P37, P72, O06

Finley, C. O01

Floto, A. P05

Flueck, D. P11

Fontana, P. P35

Forster, C. O02, O05

Franzen, D. P31

Frey, M. P27

Frey, U. O08

Fridlender, Z. P29

Froment, L. P61

Fuchs, O. O08

Funke-Chambour, M. P42, P51, P69

Furian, M. P11, P13, P15, P16, P25, P26, P47, P68

Furrer, M. P71

Fytianos, K. P08

Gaisl, T. P18, P19, P21, P22, P32, P33

Gao, Y. P57

Gasche, P. P34

Gavini, J. P51

Gazdhar, A. P08

Geiser, T. P08, P42, P51, P69

Genecand, L. P44, P46

Gex, G. P58, P70

Gianella, P. P58

Gioutsos, K. O03

Giroud, P. P36

Gonzalez, M. P37, O02, O04, O05, O06

Gorlanova, O. O08

Grange, J.C. P70

Gregoriano, C. P28

Greub, G. P01, P02, P41

Greutmann, M. P49

Grewe, F. P33

Grillet, Y. P38

Grize, L. P12, P14

Groth, A. P13, P15, P16, P25, P26

Guenat, O. O10

Guérin, S. P63

Guler, S.A. P06, P69

Güngör, T. O09

Haaksma-Herczegh, M. P29

Haba Rubio, J. P20

Hachulla, A.-L. P44, P48

Haile, S.R. P18, P22, P32, P33
Hall, S. O10

Hall, S.R.R. P57, P61

Hanna, W. O01

Hänni, B. P30

Hartmann, R. P59

Hasler, E.D. P11, P45, P47

Hasselbach, D. O04

Hayau, J. O06

Hegermann, J. P64

Heinzer, R. P20

Herschmann, S. P20

Hirsch, H. P14

Hobi, N. P51

Hostettler, K.E. P07, P09, P56

Howarth, N. P53

Huber, F. P11

Hughes, K. O01

Irani, S. P27

Jahn, K. P39, P59

Janssens, J.-P. P34, P38

Jaton, K. P01, P02, P41

Joyeux-Faure, M. P38

Jung, A. P54

Kaelin, R. P66

Kahn, P. P09

Karenovic, W. O06

Karenovics, W. P07, P50, P56

Kern, L. P28

Khan, P. P23

Kharat, A. P07, P56

Kieninger, E. P54

Kind, R. P13

Knudsen, L. P08

Kocher, G. P57, O03

Kocher, G.J. P61

Kocianova, J. P29

Kohlbrenner, D. P27

Kohler, M. P18, P19, P21, P22, P27, P32, P33

Kolb, M. P58

Koliakos, E. P70

Korten, I. O08

Kostikas, K. P12

Koutsokera, A. P03, O05

Krauss, J. P69

Krempaska, K. P51

Krueger, T. O02, O04, O05, O06

Kuehni, C. O09 
Lador, F. P44, P46, P48

Lamoth, F. P40

Latshang, T. P71

Latshang, T.D. P11

Latzin, P. P54, O08

Laurent, S. P01

Lazor, R. P52, P53, P55

Leslie, N. O04

Letovanec, I. P55, P72

Lettau, F. P21

Leuppi, J.D. P27, P28

Liang, S.-Q. P61

Lichtblau, M. P10, P11, P13, P15, P16, P25, P26, P45, $\mathrm{P} 47, \mathrm{P} 49$

Lin, D. P42

Liu, S. P57

Lopez-Hernandez, Y. O01

Louis, R. P12, P14

Lovis, A. P37, P40

Lutz, J.A. O03

Mademilov, M. P10, P13, P15, P16, P25, P26, P68

Malhotra, S. P05

Mangold, V. P28

Marazhapov, N.H. P13, P15, P16, P25, P26

Marienfeld, M. P69

Marques-Vidal, P. P20

Marsland, B. O07

Marsland, B.J. P03

Marti, T. O10

Marti, T.M. P57, P61

Martin, J. P37

Masserey, E. P41

Matula, B. P29

Mayer, L. P10, P13, P15, P16, P25, P26, P68

Mazza-Stalder, J. P01, P02, P41

Medvedchikov, A. P29

Meier, C. P25

Mercier, L. P03

Merz, L. P01

Meyer, M. P53

Michael, F. P10

Minervini, F. O01

Mitropoulou, G. P55

Miyazaki, T. O01

Moehrlen, U. O10

Montet, X. P07, P56

Morel, A. P50

Mornand, A. P63

Mucsci, J. P29

Müller, L. P54

Müller, R. P39

Muralt, L. P13, P15, P16, P25, P26
Naef, F. P53

Nawrocki, J. P68

Nibbering, B. O07

Nicod, L. P01, P02, P65, $\mathrm{O} 07$

Nicod, L.P. P03, P41, P55

Noble, S. P44

Noel, J. P50

Noirez, L. O06

Ochsenbein, A.F. P61

Opota, O. P01, P02, P41

Oppliger, A. P52

Osmonov, B. P13, P15, P16, P25, P26

Osswald, M. P18, P19, P32

Otth, M. O09

Pache, J.-C. P07, P48, P56, P58

Papakonstantinou, E. P14, P17, P24

Parkhill, J. P05

Pasche, A. P55

Pasquina, P. P34

Pattaroni, C. P03, O07

Pavlov, N. P42

Pearson, W. P05

Peitzsch, M. P22, P33

Peng, R.-W. P57, P61, O10

Pépin, J.-L. P38

Perentes, J.Y. P70, O02, O04, O06

Perentes, J.-Y. O05

Pernot, J. O07

Plojoux, J. P07, P50, P56, P58

Pöllinger, A. P42

Poncet, A. P58

Prella, M. P34, P65

Proietti, E. O08

Protazy, R. P18

Puget, E. P48

Purkabiri, K. P39

Raffoul, W. O04

Rapin, A. P03, O07

Rejmer, P. P19

Riether, C. P61

Ris, H.B. P72

Ris, H.-B. O04

Rochat, I. P63

Roeder, M. P19

Roos, M. P18

Röösli, M. O08

Rosner, L. O02

Rossi, C. P21

Rossi, R. P35
Rotas, I. P70

Roth, M. P17, P23, P24

Rothe, T. P28

Ruchonnet-Metrailler, I. P63

Saenghirunvattana, S. P60, P62, P67

Salina, A. P39

Sangen, J. P05

Sapčne, M. P38

Sauty, A. P55

Saxer, S. P10, P45, P47

Scarci, M. O01

Scheiwiller, P.M. P10, P11, P13, P15, P16, P25, P26

Schilter, D. P12, P14

Schindera, C. O09

Schindler, C. P14

Schliep, R. P08

Schmid, R. O10

Schmid, R.A. P57, P61, O03

Schmid, T. P36

Schneider, L. O01

Schneider, S. P13, P15, P16, P25, P26, P68

Schneider, S.R. P10

Schoch, O. P04, P35

Schumann, D.M. P12, P29, P39, P59

Schwarz, E.I. P22, P33, P45, P47

Shargall, Y. O01

Sheraliev, U. P10, P13, P15, P16, P25, P26

Siangproh, C. P60, P62, P67

Sievi, N.A. P22, P27, P33

Skwark, M. P05

Soccal, P.M. P07, P38, P44, P56, P58

Solą, J. P69

Somaini, G. P71

Sooronbaev, T. P10

Sooronbaev, T.M. P13, P15, P16, P25, P26, P68

Sorli, J. P29

Stach, B. P38

Stämpfli, S.F. P19

Steiner, S. P30

Stokes, A. P51

Stolz, D. P12, P14, P17, P23, P24, P39, P59

Stradling, J.R. P18

Straub, G. P31

Strobel, W. P14, P39

Suh, N. P48

Sun, Q. P23

Suris, J.-C. P65

Suwanakijboriharn, C. P62
Tamisier, R. P38

Tamm, M. P09, P12, P14, P17, P23, P24, P29, P39, P59

Tan, L. P11, P16

Tangpiroontham, P. P62

Tanner, F. P10

Tanner, F.C. P19

Thiel, S. P18, P19, P21, P22, P32, P33

Thurnheer, R. P27

Tognon, M. P48

Toma, C. P29

Tonko, S. P04

Touilloux, B. P65

Triponez, F. P50, O06

Trompette, A. O07

Tschanz, S. P30, P54

Ubags, N. O07

Ulrich, S. P10, P11, P11, P13, P15, P16, P25, P26, P45, $\mathrm{P} 47, \mathrm{P} 49, \mathrm{P} 68$

Usemann, J. O08

Valipour, A. P29

Vallée, J.-P. P44

Vassella, E. P61

Verjus, C. P69

Vernez, D. P52

Vienne, F. P34

Vienneau, D. O08

Von Garnier, C. P07, P28, P30, P56

Vongthilath, R. P48

Wang, L. O10

Wang, X. P23

Wetchanien, P. P67

Wick, P. P30

Widmer, S. P35

Winkelhofer, S. P21

Wongkhot, N. P60, P62, P67

Worsley, J. P50

Wotzkow, C. P30

Wurlod, D.-A. P03

$\mathrm{Xu}, \mathrm{D} . \mathrm{P} 61$

Yang, H. P61

Yang, Z. P61

Yerly, P. P43

Younossian, A.B. P34

Zakham, F. P01, P41

Zeinali, S. O10 